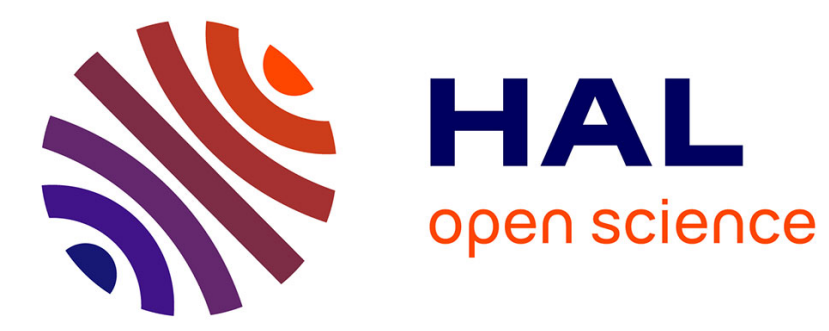

\title{
Modulation analysis for a stochastic NLS equation arising in Bose-Einstein condensation
}

\author{
Anne de Bouard, Reika Fukuizumi
}

\section{To cite this version:}

Anne de Bouard, Reika Fukuizumi. Modulation analysis for a stochastic NLS equation arising in Bose-Einstein condensation. Asymptotic Analysis, 2009, 63 (4), pp.189-235. 10.3233/ASY-20080931 . hal-00804572

\section{HAL Id: hal-00804572 \\ https://hal.science/hal-00804572}

Submitted on 25 Apr 2013

HAL is a multi-disciplinary open access archive for the deposit and dissemination of scientific research documents, whether they are published or not. The documents may come from teaching and research institutions in France or abroad, or from public or private research centers.
L'archive ouverte pluridisciplinaire HAL, est destinée au dépôt et à la diffusion de documents scientifiques de niveau recherche, publiés ou non, émanant des établissements d'enseignement et de recherche français ou étrangers, des laboratoires publics ou privés. 


\title{
MODULATION ANALYSIS FOR A STOCHASTIC NLS EQUATION ARISING IN BOSE-EINSTEIN CONDENSATION
}

\author{
ANNE DE BOUARD ${ }^{1}$ AND REIKA FUKUIZUMI ${ }^{2}$
}

\author{
${ }^{1}$ Centre de Mathématiques Appliquées, \\ CNRS et Ecole Polytechnique, 91128 Palaiseau cedex, France; \\ debouard@cmapx.polytechnique.fr \\ 2 Department of Mathematics, Hokkaido University, Sapporo 060-0810, Japan; \\ reika@math.sci.hokudai.jp
}

\begin{abstract}
We study the asymptotic behavior of the solution of a model equation for BoseEinstein condensation, in the case where the trapping potential varies randomly in time. The model is the so called Gross-Pitaevskii equation, with a quadratic potential with white noise fluctuations in time whose amplitude $\varepsilon$ tends to zero. The initial condition of the solution is a standing wave solution of the unperturbed equation. We prove that up to times of the order of $\varepsilon^{-2}$, the solution decomposes into the sum of a randomly modulated standing wave and a small remainder, and we derive the equations for the modulation parameters. In addition, we show that the first order of the remainder, as $\varepsilon$ goes to zero, converges to a Gaussian process, whose expected mode amplitudes concentrate on the third eigenmode generated by the Hermite functions, on a certain time scale.
\end{abstract}

\section{INTRODUCTION}

The first experimental realizations of Bose-Einstein condensation in weakly interacting gases sparked off many theoretical and experimental studies on coherent atomic matter. The Schrödinger equation with cubic nonlinearity and a harmonic potential has been widely used as a model equation (see for example [24]). However, magnetic trapping imposes limitations on the study of Bose-Einstein condensates, because only the weak-field seeking atomic states are confined, which may cause a chain of drawbacks (see [28]). Such problems are avoided if Bose-Einstein condensation is achieved in an optical trap based on the optical dipole force which confines atoms in all hyperfine states. The authors in [28] succeeded to obtain condensation in all-optical far-off-resonance laser trap. The use of optical traps may bring other advantages such as obtaining different geometrical configurations or creating more dense condensates. On the other hand, in real situation, one should take into account stochasticity in the dynamical behavior of the condensate, for the reason that some fluctuations of the laser intensity are observed in the experiments. Those fluctuations may be regarded as fluctuations of the harmonic trap potential in the mean field approximation (see [1]). In this case, one may be led to consider the following

1991 Mathematics Subject Classification. 35Q55, 60H15 .

Key words and phrases. Nonlinear Schrödinger equation, stochastic partial differential equations, white noise, harmonic potential, standing waves, expected mode powers. 
nonlinear Schrödinger equation (radially symmetric 2D Gross-Pitaevskii equation) perturbed by a random quadratic potential:

$$
i \frac{\partial}{\partial t} u=-\frac{1}{r} \frac{\partial}{\partial r} r \frac{\partial u}{\partial r}+(1+\varepsilon \dot{\xi}(t)) r^{2} u-\lambda|u|^{2} u-i \gamma u
$$

where $\lambda= \pm 1$ and $\dot{\xi}$ is a white noise in time with correlation function $\mathbb{E}(\dot{\xi}(t) \dot{\xi}(s))=\delta_{0}(t-s)$. Here, $\delta_{0}$ denotes the Dirac measure at the origin, $\gamma \geq 0$ and $\varepsilon>0$. The product arising in the right hand side is interpreted in the Stratonovich sense, since the noise here naturally arises as the limit of processes with nonzero correlation length. We moreover assume that the noise is real valued. The term $\varepsilon \dot{\xi}(t)$ represents the deviations of the laser intensity $E(t)$ around its mean value (see [1]). Also, in this model, the sign of $\lambda$ is related to the sign of the atomic scattering length, which may be positive or negative. A similar model was used in [12] in dimension three, except that the fluctuations there were not assumed to be delta-correlated. Related equations may also be found in the context of optic fibers. In [2] e.g., equation (1.1) without the harmonic potential term, and simply with a multiplicative noise was considered as a model for optical soliton propagation in fibers with random inhomogeneities. In [1], the qualitative properties of solutions of (1.1) is studied by using the "moments method" which consists in finding (finite dimensional) evolution equations satisfied by a few integral quantities of the solutions, like e.g., energy, momentum, and so on. A closed system of equations is found in the case where there is no damping. The solutions of this system of stochastic differential equations are then formally approximated in the limit where the noise tends to zero.

Our aim in this paper is, as a sequel to the mathematical study in [8], to investigate the influence of random perturbations on the propagation of deterministic standing waves. The method we will use, so called collective coordinate approach, consists in writing that the main part of the solution is given by a modulated soliton and in finding then the modulation equations for the soliton parameters. Such ideas to analyze the asymptotic behavior have been used by many authors in the physics literature, as well as in the study of mathematical problems (see for example, Weinstein [30], Jonsson et al $[15,16])$. The modulation theory, in general, provides an approximate and constructive answer to questions concerning the location of the standing wave and the behavior of its phase for $t>0$.

In order to state precisely the problem and our results, we consider a probability space $(\Omega, \mathcal{F}, \mathbb{P})$ endowed with a standard filtration $\left(\mathcal{F}_{t}\right)_{t \geq 0}$ and a standard real valued Brownian motion $W(t)$ on $\mathbb{R}^{+}$associated with the filtration $\left(\mathcal{F}_{t}\right)_{t \geq 0}$. We set $\dot{\xi}=\frac{d W}{d t}$ and then consider the stochastic nonlinear Schrödinger equation:

$$
i d u+\left(\Delta u-|x|^{2} u+\lambda|u|^{2 \sigma} u+i \gamma u\right) d t=\varepsilon|x|^{2} u \circ d W,
$$

where $\circ$ stands for a Stratonovich product in the right hand side of (1.2), $\sigma>0, \varepsilon>0, \gamma \geq 0$ and $\lambda= \pm 1$. We will use the equivalent Itô equation which may be written as

$$
i d u+\left(\Delta u-|x|^{2} u+\frac{i}{2} \varepsilon^{2}|x|^{4} u+\lambda|u|^{2 \sigma} u+i \gamma u\right) d t=\varepsilon|x|^{2} u d W .
$$

Moreover, we do not restrict ourselves to dimension two here, and consider that in Eq. (1.2) or (1.3), $x \in \mathbb{R}^{d}, d=1$ or 2 . 
Let us give some notations. For $p \geq 1, L^{p}\left(\mathbb{R}^{d}\right)$ is the Lebesgue space of complex valued, $p$-th summable functions, and the inner product in the Hilbert space $L^{2}\left(\mathbb{R}^{d}\right)$ is denoted by $(\cdot, \cdot)$, i.e.,

$$
(u, v)=\int_{\mathbb{R}^{d}} u(x) \overline{v(x)} d x, \quad \text { for } \quad u, v \in L^{2}\left(\mathbb{R}^{d}\right) .
$$

Moreover $\langle u, v\rangle=\operatorname{Re}(u, v)$. The norm in $L^{p}\left(\mathbb{R}^{d}\right)$ is denoted by $|\cdot|_{L^{p}}$. With the aim of studying the spectrum of linearized operator we will consider the space $\mathbb{L}^{2}\left(\mathbb{R}^{d}\right)$ of $\mathbb{R}^{2}$-valued functions of $L^{2}\left(\mathbb{R}^{d}\right) \times L^{2}\left(\mathbb{R}^{d}\right)$, and $\tilde{\mathbb{L}}^{2}\left(\mathbb{R}^{d}\right)=\mathbb{L}^{2}\left(\mathbb{R}^{d}\right)+i \mathbb{L}^{2}\left(\mathbb{R}^{d}\right)$, which is identified with the space of $\mathbb{C}^{2}$-valued, square integrable functions. $\tilde{\mathbb{L}}^{2}\left(\mathbb{R}^{d}\right)$ is endowed with inner product

$$
((\mathcal{U}, \mathcal{V}))=\left(u_{1}, v_{1}\right)+\left(u_{2}, v_{2}\right), \quad|\mathcal{U}|_{\tilde{\mathbb{L}}^{2}\left(\mathbb{R}^{d}\right)}^{2}=((\mathcal{U}, \mathcal{U}))
$$

where

$$
\mathcal{U}\left(\begin{array}{l}
u_{1} \\
u_{2}
\end{array}\right), \quad \mathcal{V}\left(\begin{array}{l}
v_{1} \\
v_{2}
\end{array}\right) \in \tilde{\mathbb{L}}^{2}\left(\mathbb{R}^{d}\right)
$$

Operator norms will be denoted by $\|\cdot\|_{\mathcal{L}(X)}$ or $\|\cdot\|_{\mathcal{L}(X, Y)}$, if $X$ and $Y$ are Banach spaces where the operators are defined.

We define for $s \in \mathbb{R}$ the space $H^{s}\left(\mathbb{R}^{d}\right)$ of tempered distributions $v \in \mathcal{S}^{\prime}\left(\mathbb{R}^{d}\right)$ whose Fourier transform $\hat{v}$ satisfies $\left(1+|\xi|^{2}\right)^{s / 2} \hat{v} \in L^{2}\left(\mathbb{R}^{d}\right)$. The norm in $H^{s}\left(\mathbb{R}^{d}\right)$ is denoted by $|\cdot|_{H^{s}}$. We denote the weighted space $\left\{v \in H^{1}\left(\mathbb{R}^{d}\right) ;|x| v \in L^{2}\left(\mathbb{R}^{d}\right)\right\}$ by $\Sigma$ and its norm by $|\cdot|_{\Sigma}=\left(|\cdot|_{H^{1}}^{2}+|x \cdot|_{L^{2}}^{2}\right)^{1 / 2}$.

We define the energy

$$
H(u)=\frac{1}{2}|\nabla u|_{L^{2}}^{2}+\frac{1}{2}|x u|_{L^{2}}^{2}-\frac{\lambda}{2 \sigma+2}|u|_{L^{2 \sigma+2}}^{2 \sigma+2},
$$

which is a conserved quantity of the deterministic equation without damping, i.e., (1.2) with $\varepsilon=0$ and $\gamma=0$. We will consider solutions in the space $\Sigma$, which is the natural space where $H$ is well defined, thanks to the embedding $\Sigma \subset H^{1}\left(\mathbb{R}^{d}\right) \subset L^{2 \sigma+2}\left(\mathbb{R}^{d}\right)$, for $\sigma<\frac{2 d}{d-2}$ if $d \geq 3$ or $\sigma<+\infty$ if $d=1,2$.

In the case where $\varepsilon=0$ and $\gamma=0$, it is known that in the energy space $\Sigma$, equation (1.2) is locally well posed for $\lambda= \pm 1, \sigma<\frac{2 d}{d-2}$ if $d \geq 3$ or $\sigma<+\infty$ if $d=1,2$ and globally well posed if either $\lambda=-1$ or $\lambda=1$ and $\sigma<2 / d$ (see [21]). Also, blow up phenomena appear for $\lambda=1$ and $\sigma \geq 2 / d$ under certain condition on the initial data, for example, a data with negative energy (see [3]). We generalized in [8] these deterministic results to equation (1.2) and we also studied the local existence of solutions in dimensions $d=1$ or 2 .

Theorem 1. ([8]) Assume $\sigma>0, \gamma \geq 0$ and $\lambda= \pm 1$. Assume $u_{0} \in \Sigma$ if $d=1$, or $u_{0} \in \Sigma^{2}$ and $1 / 2 \leq \sigma \leq 1$ if $d=2$. Then there exist a stopping time $\tau^{*}\left(u_{0}, \omega\right)$ and a unique solution $u(t)$ adapted to $\left(\mathcal{F}_{t}\right)_{t \geq 0}$ of $(1.2)$ with $u(0)=u_{0}$, which is almost surely in $C([0, \tau] ; \Sigma)$ for any $\tau<\tau^{*}\left(u_{0}\right)$. Moreover, we have almost surely,

$$
\tau^{*}\left(u_{0}, \omega\right)=+\infty \text { or } \limsup _{t / \tau^{*}\left(u_{0}, \omega\right)}|u(t)|_{\Sigma}=+\infty .
$$


Here, for $m \in \mathbb{N}, m \geq 1$,

$$
\Sigma^{m}=H^{m} \cap\left\{u \in L^{2}\left(\mathbb{R}^{d}\right) ;\left(1+|x|^{2}\right)^{m / 2} u \in L^{2}\left(\mathbb{R}^{d}\right)\right\} \subset \Sigma .
$$

The dual space of $\Sigma^{m}$ in the $L^{2}$ sense, which we denote by $\Sigma^{-m}$, is

$$
\Sigma^{-m}=H^{-m}+\left\{u \in \mathcal{S}^{\prime}\left(\mathbb{R}^{d}\right) ; \frac{u}{\left(1+|x|^{2}\right)^{m / 2}} \in L^{2}\left(\mathbb{R}^{d}\right)\right\}
$$

and the norm in $\Sigma^{-m}$ is given by

$$
|u|_{\Sigma^{-m}}=\inf \left\{\left|u_{1}\right|_{H^{-m}}+\left|\frac{u_{2}}{\left(1+|x|^{2}\right)^{m / 2}}\right|_{L^{2}} ; u=u_{1}+u_{2}\right\} \quad \text { for } \quad u \in \Sigma^{-m} .
$$

The $\Sigma^{2}$ regularity for the initial data in the case $d=2$ is required for the energy equality, and in order to get pathwise continuous solutions with values in $\Sigma$ : the solution given by Theorem 1 is a strong solution in the probabilistic sense. ¿From now on, we fix $\lambda=1$ and $\gamma=0$, so that we consider the equation

$$
i d u+\left(\Delta u-|x|^{2} u+\frac{i}{2} \varepsilon^{2}|x|^{4} u+|u|^{2 \sigma} u\right) d t=\varepsilon|x|^{2} u d W .
$$

We now go back to the deterministic case and consider the two parameter family of standing wave solutions

$$
e^{i(\mu t+\theta)} \phi_{\mu}(x)
$$

of equation (1.2) with $\varepsilon=0$ and $\theta, \mu \in \mathbb{R}$, i.e.,

$$
i \partial_{t} u+\Delta u-|x|^{2} u+|u|^{2 \sigma} u=0, \quad x \in \mathbb{R}^{d}, \quad t \geq 0 .
$$

The standing wave solution satisfies (1.7) if and only if $\phi_{\mu}$ satisfies the following semilinear elliptic equation:

$$
-\Delta \phi+|x|^{2} \phi+\mu \phi-|\phi|^{2 \sigma} \phi=0, \quad x \in \mathbb{R}^{d} .
$$

The existence of the standing wave solutions is proved, with the help of the compact embedding $\Sigma \subset L^{2}$, for any $\mu>-\lambda_{0}$ where

$$
\lambda_{0}=\inf \left\{|\nabla v|_{L^{2}}^{2}+|x v|_{L^{2}}^{2} ; v \in \Sigma,|v|_{L^{2}}=1\right\}=d .
$$

The inverse scattering method gives some qualitative properties (e.g. asymptotic stability) for this type of solitary waves for completely integrable systems. However integrability is restricted to $1 \mathrm{D}$ cubic nonlinear Schrödinger equation without any potential term and does not apply to (1.7). Stability properties of such solutions in non-integrable case have also been the object of several studies, beginning with Cazenave and Lions [4], Weinstein [31], and Grillakis Shatah and Strauss [14]. For the specific equation (1.8) with a harmonic potential, there have been some studies on the orbital stability; see for example, Rose and Weinstein [26], the second author and Ohta [10]. Note that together with the energy (1.4), another conserved quantity for equation (1.7) is given by

$$
Q(u)=\frac{1}{2}|u|_{L^{2}}^{2}
$$


namely, we have $Q(u(t))=Q(u(0))$ for any solution $u \in C([0, T] ; \Sigma)$ of (1.7), and Eq.(1.8) can be written as $H^{\prime}\left(\phi_{\mu}\right)+\mu Q^{\prime}\left(\phi_{\mu}\right)=0$. The proof of orbital stability is based on the use of the functional

$$
S_{\mu}(u)=H(u)+\mu Q(u), \quad u \in \Sigma,
$$

as a Lyapunov functional. In order to show the positivity of the second derivative of this Lyapunov functional, the positivity of the following linearized operator

$$
L_{\mu}^{-}=-\Delta+|x|^{2}+\mu-(2 \sigma+1) \phi_{\mu}^{2 \sigma}
$$

is essential. It appears that $S_{\mu}^{\prime \prime}\left(\phi_{\mu}\right)$ is positive when restricted to the subspace of $\Sigma$ of functions orthogonal in $L^{2}$ to both $\phi_{\mu}$ and $i \phi_{\mu}$ provided $\mu$ is sufficiently close to $-\lambda_{0}$ and $\sigma>0$, or $\mu>0$ is sufficiently large and $\sigma \leq 2 / d$. This implies that the set $\left\{e^{i \theta} \phi_{\mu}, \theta \in \mathbb{R}\right\}$ is a set of local minimizers of $S_{\mu}$ restricted to the manifold $\left\{u \in \Sigma, Q(u)=Q\left(\phi_{\mu}\right)\right\}$.

Another condition which gives the positivity of $S_{\mu}^{\prime \prime}\left(\phi_{\mu}\right)$,

$$
\partial_{\mu}\left|\phi_{\mu}\right|_{L^{2}}^{2}>0
$$

is often used (see, e.g., $[14,26])$. The scaling invariance of the equation for the standing waves allows, in general, to check whether (1.13) is satisfied for general $\mu$; however we cannot expect this scaling property here due to the harmonic potential. Thus it is natural to consider frequencies $\mu$ which are close to $-\lambda_{0}$ and make use of the properties of spectrum and solutions of linear problems that are already known. Recall that the linear eigenvalue problem

$$
-\Delta u+|x|^{2} u=\lambda u, \quad \lambda \in \mathbb{R}
$$

consists only of discrete eigenvalues $\lambda_{k}(k \in \mathbb{N} \cup\{0\})$ and the associated eigenfunctions are the Hermite functions (see [29]). Thus the bifurcation argument near $\mu=-\lambda_{0}$ is effective, which is also another method to ensure the existence of bound state solutions of (1.8) (see Kurth [19]). We will consider only the ground state $\phi_{\mu}$ of (1.8) in this paper. Namely $\phi_{\mu}$ is the unique positive radial solution of (1.8) (see $\mathrm{Li}$ and $\mathrm{Ni}$ [20] for the radial symmetry, Kabeya and Tanaka [17] for the uniqueness). For (1.7), it was verified in [10] that there exists $\mu^{*}$ such that for any $\mu \in\left(-\lambda_{0}, \mu^{*}\right)$ the positivity of $S_{\mu}^{\prime \prime}\left(\phi_{\mu}\right)$ holds under the above suitable orthogonality conditions for any $\sigma>0$. We summarize here the properties of $\phi_{\mu}$ that we will use later.

Proposition 1. Let $d=1$ or $2,0<\sigma<+\infty$ and $\mu>-\lambda_{0}$. Let $\phi_{\mu}$ be the unique positive radial solution of (1.8).

(i) $\mu \mapsto \phi_{\mu}$ is a $C^{1}$ mapping from $\left(-\lambda_{0},+\infty\right)$ to $\Sigma^{2}$ ( and $\Sigma^{4}$ if $\sigma \geq 1 / 2$ ), moreover it is a $C^{2}$ mapping if $\sigma \geq 1 / 2$.

(ii) There is a $\mu_{*}>-\lambda_{0}$ such that for any $\mu \in\left(-\lambda_{0}, \mu_{*}\right)$ there exist $\nu=\nu(\mu)>0$, for any $v \in \Sigma$ satisfying $\operatorname{Re}\left(v, \phi_{\mu}\right)=\operatorname{Re}\left(v, i \phi_{\mu}\right)=0$, we have

$$
\left\langle S_{\mu}^{\prime \prime}\left(\phi_{\mu}\right) v, v\right\rangle \geq \nu|v|_{\Sigma}^{2}
$$

(iii) $\left(\phi_{\mu}, \partial_{\mu} \phi_{\mu}\right)$ is strictly positive for $\mu \in\left(-\lambda_{0}, \mu^{*}\right)$.

(iv) $\phi_{\mu} \in \bigcap_{2 \leq q<\infty} W^{2, q} \cap C^{2}$.

(v) For any $\mu_{1}>0$, there are positive constants $C_{0}, C_{1}$, depending on $\mu_{1}$, such that for all $\mu \in\left(-\lambda_{0}, \mu_{1}\right)$, the inequality $\left|\phi_{\mu}(x)\right| \leq C_{0} e^{-C_{1}|x|^{2}}$ holds, for all $x \in \mathbb{R}^{d}$. 
We will give a few references concerning the proof of this proposition in Section 6 .

The linearization problem around $e^{i \mu t} \phi_{\mu}$ in (1.7) is precisely written as

where

$$
\frac{d y}{d t}=J \mathcal{L}_{\mu} y \quad \text { in } \quad \Sigma^{-1}
$$

$$
\begin{gathered}
J=-i:\left(\begin{array}{c}
\operatorname{Re} u \\
\operatorname{Im} u
\end{array}\right) \mapsto\left(\begin{array}{cc}
0 & 1 \\
-1 & 0
\end{array}\right)\left(\begin{array}{c}
\operatorname{Re} u \\
\operatorname{Im} u
\end{array}\right) \\
\mathcal{L}_{\mu}=S_{\mu}^{\prime \prime}\left(\phi_{\mu}\right)\left(\begin{array}{cc}
L_{\mu}^{-} & 0 \\
0 & L_{\mu}^{+}
\end{array}\right), \\
L_{\mu}^{+}=-\Delta+|x|^{2}+\mu-\phi_{\mu}^{2 \sigma},
\end{gathered}
$$

$L_{\mu}^{-}$is already defined in (1.12) above. Note that the operator $L_{\mu}^{+}$is nonnegative since $\phi_{\mu}$ is a positive solution of Eq.(1.8). Note also that

$$
\left\{\begin{array}{l}
L_{\mu}^{+} \phi_{\mu}=0 \\
L_{\mu}^{-} \partial_{\mu} \phi_{\mu}=-\phi_{\mu} ;
\end{array}\right.
$$

more precisely, $J \mathcal{L}_{\mu}$ has a two dimensional generalized null-space spanned by

$$
\left(\begin{array}{c}
\partial_{\mu} \phi_{\mu} \\
0
\end{array}\right), \quad\left(\begin{array}{c}
0 \\
\phi_{\mu}
\end{array}\right)
$$

and the rest of spectrum is purely discrete on the imaginary axis for the frequencies $\mu$ close to $-\lambda_{0}$. We will study this linearized problem in details in Section 6 below, regarding $J \mathcal{L}_{\mu}$ as a perturbed operator from $J \mathcal{L}_{-\lambda_{0}}$, where

$$
J \mathcal{L}_{-\lambda_{0}}\left(\begin{array}{cc}
0 & 1 \\
-1 & 0
\end{array}\right)\left(\begin{array}{cc}
\Xi & 0 \\
0 & \Xi
\end{array}\right)\left(\begin{array}{cc}
0 & \Xi \\
-\Xi & 0
\end{array}\right)
$$

and $\Xi=-\Delta+|x|^{2}-\lambda_{0}$. This kind of analysis was used in Pelinovsky and Kevrekidis [23] where the spectrum of linearized operators around the standing wave solution are investigated using, similarly, a bifurcation analysis and a regular perturbation method.

Concerning results related to the asymptotic behavior of solutions of (1.7) starting from the standing wave solution (1.6), Jonsson, Fröhlich, Gustafson and Sigal [15, 16] analysed the modulation equation, assuming that the effect of the harmonic potential term is sufficiently small.

Our purpose here is to investigate the influence of random perturbations of the form given in equation (1.5) on the phase and the frequency of standing wave solutions (1.6). We consider the solution $u^{\varepsilon}(t, x)$ of equation (1.5), given by Theorem 1 , and with $u^{\varepsilon}(0, x)=\phi_{\mu_{0}}(x)$ where $\mu_{0}$ is fixed such as $\mu_{0} \in\left(-\lambda_{0}, \mu^{*}\right)$. We may expect that, if $\varepsilon$ is small, the main part of the solution is a standing wave, randomly modulated in its phase $\theta$ and frequency $\mu$. We will briefly comment in Section 3 that this is true for time less than $\varepsilon^{-2}$, following the proof of the same kind of result by the first author and Debussche in [6] for the Korteweg-de Vries equation with an additive noise. 
Next, we study more precisely the behavior at order one in $\varepsilon$ of the remaining term in the preceding decomposition as $\varepsilon$ goes to zero. The preceding decomposition says that the solution $u^{\varepsilon}(t, x)$ is written as

$$
u^{\varepsilon}(t, x)=e^{i \theta^{\varepsilon}(t)}\left(\phi_{\mu^{\varepsilon}(t)}(x)+\varepsilon \eta^{\varepsilon}(t, x)\right)
$$

where $\theta^{\varepsilon}(t)$ and $\mu^{\varepsilon}(t)$ are the modulation parameters; these are semi-martingale processes defined up to times of the order of $\varepsilon^{-2}$. That means that the shape of the standing wave is preserved over this time scale. We will show that the process $\eta^{\varepsilon}$ converges as $\varepsilon$ goes to zero, in probability, to a Gaussian process $\eta$. Moreover, $\theta^{\varepsilon}(t)$ and $\mu^{\varepsilon}(t)$ can be developed up to order one in $\varepsilon$, and we get

$$
\left\{\begin{array}{l}
d \mu^{\varepsilon}(t)=o(\varepsilon), \\
d \theta^{\varepsilon}(t)=\mu_{0} d t-\varepsilon \frac{\left(|x|^{2} \phi_{\mu_{0}}, \partial_{\mu} \phi_{\mu_{0}}\right)}{\left(\phi_{\mu_{0}}, \partial_{\mu} \phi_{\mu_{0}}\right)} d W+o(\varepsilon) .
\end{array}\right.
$$

This shows in particular that at first order the noise does not act on the frequency of the standing wave, but only on its phase.

Finally we investigate the behavior of the process $\eta$ as $t$ goes to $+\infty$, in the case $\sigma>1$. We study in Section 6 the distribution of the mode powers of $\eta$, i.e., $\mathbb{E}\left(\left|\eta_{k}(t)\right|^{2}\right)$ for each $k \in$ $\mathbb{N} \cup\{0\}$, when the frequency $\mu_{0}$ is sufficiently close to $-\lambda_{0}$. Here, $\eta_{k}$ is the component of $\eta$ on the $k$-th eigenfunction of $-\Delta+|x|^{2}$ (recall that the family of those eigenfunctions forms a complete orthonormal system in $\left.L^{2}\left(\mathbb{R}^{d}\right)\right)$. Specifically, on a time scale of order of $\left(\mu_{0}+\lambda_{0}\right)^{-1 / \sigma}$, one can expect the power to be concentrated mainly in the third mode. This observation is actually inspired by Papanicolaou [22], Kirr and Weinstein [18]. The authors in [22, 18] treated a system perturbed by a multiplicative random potential with a small parameter $\kappa$ describing the amplitude of the random potential. In the limit $t \rightarrow+\infty, \kappa \rightarrow 0, \kappa^{2} t=$ constant (at least in their case), the mode powers satisfy a system of coupled equations which are called master equations. In this context, the question of how the mode powers evolve with $t$ is of fundamental interest. We also derive our reduced master equation which explains the mode-power concentration.

The paper is organized as follows: in Section 2, we state precisely our results. In Section 3 , we justify the existence of the modulation parameters and we give an estimate on the time up to which the modulation procedure is available. In Section 4 we give the equations of the modulation parameters. Section 5 is devoted to estimates on the remainder term whose most technical parts are postponed to Section 7. Using these estimates, we will also show the convergence as $\varepsilon$ goes to zero. Section 6 is devoted to analyze the drift part of the limit equation where we will use a bifurcation and a perturbation method from the linear eigenvalue problem for $\mu_{0}$ close to $-\lambda_{0}$. The mode-power concentration will also be proved in Section 6 , deriving the master equation. To lighten notations, we denote sometimes in what follows by $C(\alpha, \cdots)$ a constant which depends on $\alpha$ and so on.

\section{MAIN RESULTS}

We fix $\mu_{0} \in\left(-\lambda_{0}, \mu^{*}\right)$ and consider for $\varepsilon>0$ the solution $u^{\varepsilon}(t, x)$ of equation (1.5) given by Theorem 1 with initial data $u^{\varepsilon}(0, x)=\phi_{\mu_{0}}(x)$.

The first theorem says that we can decompose $u^{\varepsilon}$ as the sum of a modulated standing wave and a remainder with small $\Sigma$ norm, for $t$ less than some stopping time $\tau^{\varepsilon}$, and that this $\tau^{\varepsilon}$ goes 
to infinity in probability as $\varepsilon$ goes to zero. We will see then that the remaining part is of order one with respect to $\varepsilon$. The proof of the theorem is rather similar to those in $[6,7]$, but for the sake of completeness we repeat it in the next section. We remark that the proof of theorems 2 and 3 will be completed in subsection 6.3 (see Remark 2.1 below). This decomposition is in the form

$$
u^{\varepsilon}(t, x)=e^{i \theta^{\varepsilon}(t)}\left(\phi_{\mu^{\varepsilon}(t)}(x)+\varepsilon \eta^{\varepsilon}(t, x)\right)
$$

for some semi-martingale processes $\theta^{\varepsilon}(t), \mu^{\varepsilon}(t)$ with values in $\mathbb{R}$, and $\eta^{\varepsilon}$ with value in $\Sigma$. We note that the expression of the main part of the solution is not unique, neither are the modulation parameters. They depend on the choice of some specific conditions on the remaining part. For instance, in order to obtain the simple equation (1.18) for $\theta^{\varepsilon}$, the spectral projection of the remaining part of the solution on the generalized nullspace of $J \mathcal{L}_{\mu_{0}}$ must be zero, at least at order one in $\varepsilon$. However, in order to estimate the exit time, it is more convenient to use the orthogonality of the remaining part to the nullspace of $\mathcal{L}_{\mu_{0}}$, since it ensures the positivity of $S_{\mu_{0}}^{\prime \prime}\left(\phi_{\mu_{0}}\right)$. This is why we do not state precisely the orthogonality conditions in the following Theorem 2 (see Remark 2.1 below).

Theorem 2. Assume $d=1$ and $1 / 2 \leq \sigma$, or $d=2$ and $1 / 2 \leq \sigma \leq 1$. Let $\mu_{0} \in\left(-\lambda_{0}, \mu^{*}\right)$ be fixed. For $\varepsilon>0$, let $u^{\varepsilon}(t, x)$, as defined above, be the solution of $(1.5)$ with $u(0, x)=\phi_{\mu_{0}}(x)$. Then there exists $\alpha_{0}>0$ such that, for each $\alpha, 0<\alpha \leq \alpha_{0}$, there is a stopping time $\tau_{\alpha}^{\varepsilon} \in\left(0, \tau^{*}\left(\phi_{\mu_{0}}\right)\right)$ a.s., and there are semi-martingale processes $\tilde{\mu}^{\varepsilon}(t)$ and $\tilde{\theta}^{\varepsilon}(t)$, defined a.s. for $t \leq \tau_{\alpha}^{\varepsilon}$, with values in $\mathbb{R}$, so that if we set $\varepsilon \tilde{\eta}^{\varepsilon}(t, x)=e^{-i \tilde{\theta}^{\varepsilon}(t)} u^{\varepsilon}(t, x)-\phi_{\tilde{\mu}^{\varepsilon}(t)}(x)$, then, a.s. for $t \leq \tau_{\alpha}^{\varepsilon}$,

$$
\left|\tilde{\mu}^{\varepsilon}(t)-\mu_{0}\right| \leq \alpha
$$

and

$$
\left|\varepsilon \tilde{\eta}^{\varepsilon}(t)\right|_{\Sigma} \leq \alpha
$$

In addition, there is a constant $C=C\left(\alpha, \mu_{0}\right)>0$, such that for any $T>0$ and any $\alpha \leq \alpha_{0}$, there is an $\varepsilon_{0}>0$, such that for each $\varepsilon<\varepsilon_{0}$,

$$
\mathbb{P}\left(\tau_{\alpha}^{\varepsilon} \leq T\right) \leq \exp \left(-\frac{C}{\varepsilon^{2} T}\right) .
$$

Remark 2.1. For the proof of the estimate (2.4), the following orthogonality conditions will be used :

and

$$
\operatorname{Re}\left(\eta^{\varepsilon}, \phi_{\mu_{0}}\right)=0, \quad \text { a.s., } \quad t \leq \tau^{\varepsilon},
$$

$$
\operatorname{Re}\left(\eta^{\varepsilon}, i \phi_{\mu_{0}}\right)=0, \quad \text { a.s. }, \quad t \leq \tau^{\varepsilon}
$$

where $\tau^{\varepsilon}$ is the same stopping time as in Theorem 1. Hence, we first use these conditions on the remaining part in Section 3, in order to define $\eta^{\varepsilon}$, and in Section 6 we make a change of the modulation parameters which allows to get a new decomposition (with the same stopping time $\left.\tau^{\varepsilon}\right)$, satisfying at order one in $\varepsilon$ the simple equation Eq. (1.18).

Remark 2.2. Attention is given to the upper bound (2.4) where the product $\varepsilon^{2} T$ appears. From the theorem, we can expect, with high probability, that the solution of (1.2) stays in a neighborhood of the randomly modulated standing wave at least for times small compared to $\varepsilon^{-2}$. Whether this time scale of $\varepsilon^{-2}$ is optimal or not still leaves a room for discussion in our case. In [9], the authors considered the same exit problem for Korteweg-de Vries equation with an additive noise. An exponential lower bound of the same order in the parameters $T$ and $\varepsilon$ 
as the upper bound is proved in [9], which ensures that the typical time scale on which the solution remains in the neighborhood of the modulated soliton is indeed $\varepsilon^{-2}$. The proof of such a lower bound requires the use of a Large Deviation Principle together with the resolution of a control problem, which allows to get an upper bound on the rate function. The proof of the LDP is far from obvious in our case since we cannot solve equation (1.5) by a contraction argument; moreover the control problem is a control problem by a potential; as far as we know, the nonlinear controllability problems by a time dependent potential is an open problem.

Next the following result is concerned with the analysis of the behavior of $\eta^{\varepsilon}$, and of the modulation parameters as $\varepsilon$ goes to zero.

Theorem 3. Assume $d=1$ and $1 \leq \sigma$, or $d=2$ and $\sigma=1$. Let $\mu_{0} \in\left(-\lambda_{0}, \mu^{*}\right)$ be fixed and $\tilde{\eta}^{\varepsilon}$, $\tilde{\theta}^{\varepsilon}, \tilde{\mu}^{\varepsilon}$, for $\varepsilon>0$ be given by Theorem 2, with $\alpha \leq \alpha_{0}$ fixed. Then, for any $T>0$, the process $\left(\tilde{\eta}^{\varepsilon}(t)\right)_{t \in\left[0, T \wedge \tau_{\alpha}^{\varepsilon}\right]}$ converges in probability, as $\varepsilon$ goes to zero, to a process $\tilde{\eta}$ satisfying

$$
d \tilde{\eta}=J \mathcal{L}_{\mu_{0}} \tilde{\eta} d t-\left(I-P_{\mu_{0}}\right)\left(\begin{array}{c}
0 \\
|x|^{2} \phi_{\mu_{0}}
\end{array}\right) d W
$$

with $\tilde{\eta}(0)=0$, where $P_{\mu_{0}}$ is the spectral projection onto the generalized null space of $J \mathcal{L}_{\mu_{0}}$. The convergence holds in $C\left(\left[0, \tau_{\alpha}^{\varepsilon} \wedge T\right], L^{2}\right)$.

The above process $\tilde{\eta}$ satisfies for any $T>0$ the estimate

$$
\mathbb{E}\left(\sup _{t \leq T}|\tilde{\eta}(t)|_{\Sigma}^{2}\right) \leq C T
$$

for some constant $C>0$.

Moreover the modulation parameters may be written, for $t \leq \tau_{\alpha}^{\varepsilon}$, as

$$
d \tilde{\theta}^{\varepsilon}=\tilde{\mu}^{\varepsilon} d t+\varepsilon \tilde{y}^{\varepsilon} d t+\varepsilon \tilde{z}^{\varepsilon} d W
$$

and

$$
d \tilde{\mu}^{\varepsilon}=\varepsilon \tilde{a}^{\varepsilon} d t+\varepsilon \tilde{b}^{\varepsilon} d W
$$

for some adapted processes $\tilde{y}^{\varepsilon}$, $\tilde{a}^{\varepsilon}, \tilde{z}^{\varepsilon}$, $\tilde{b}^{\varepsilon}$ with values in $\mathbb{R}$ satisfying: as $\varepsilon$ goes to zero, $\tilde{a}^{\varepsilon}$, $\tilde{b}^{\varepsilon}, \tilde{y}^{\varepsilon}$ converge to 0 in probability in $C([0, T])$, while $\tilde{z}^{\varepsilon}$ converges in probability in $C([0, T])$ to $-\left(\partial_{\mu} \phi_{\mu_{0}}, \phi_{\mu_{0}}\right)^{-1}\left(\partial_{\mu} \phi_{\mu_{0}},|x|^{2} \phi_{\mu_{0}}\right)$.

At last, we derive the following theorem concerning the asymptotic behaviour of $\tilde{\eta}(t, x)$ for large $t>0$. As mentioned in the introduction, the operator $J \mathcal{L}_{\mu_{0}}$ may be regarded as a perturbation from $J \mathcal{L}_{-\lambda_{0}}$. Let us write the equation as

$$
d \tilde{\eta}=\left(J \mathcal{L}_{-\lambda_{0}}+B_{\mu_{0}}\right) \tilde{\eta} d t-\left(I-P_{\mu_{0}}\right)\left(\begin{array}{c}
0 \\
|x|^{2} \phi_{\mu_{0}}
\end{array}\right) d W
$$

and consider this equation as perturbation of the system

$$
d \tilde{\eta}=J \mathcal{L}_{-\lambda_{0}} \tilde{\eta} d t
$$

by the small bounded operator $B_{\mu_{0}}$ plus an additive noise with small amplitude as $\mu_{0}$ tends to $-\lambda_{0}$. The system (2.11) can be studied by decomposing the initial state on the complete system of eigenstates of $J \mathcal{L}_{-\lambda_{0}}$. When (2.11) is perturbed, then the system of ODE's becomes an infinite coupled system of equations, and the behavior of its solutions may be rather complex. 
We get the following result on the evolution of the average power in the $k$-th mode of $\eta$. This result is restricted to the one dimensional case, because it requires the condition $\sigma>1$ (see Remark 2.4) below.

Let us denote by $\tilde{\lambda}_{k}=\lambda_{k}-\lambda_{0}$ the eigenvalues of the operator $-\Delta+|x|^{2}-\lambda_{0}$ in 1D, and let

$$
\Phi_{k}(x)=\frac{1}{\left(2^{k} k !\right)^{1 / 2} \pi^{1 / 4}} H_{k}(x) e^{-|x|^{2} / 2}
$$

be the corresponding eigenfunction for $k \in \mathbb{N} \cup\{0\}, H_{k}(x)$ being the Hermite polynomials. We will see that $J \mathcal{L}_{-\lambda_{0}}$ has purely discrete eigenvalues denoted by $\xi_{k}^{ \pm}= \pm i \tilde{\lambda}_{k}$ whose associated eigenfunctions may be choosen as

$$
\vec{\Phi}_{k}^{ \pm}\left(\begin{array}{c} 
\pm i \Phi_{k} \\
\mp \Phi_{k}
\end{array}\right), \text { for } k \geq 1, \quad \vec{\Phi}_{0}^{-}\left(\begin{array}{c}
\Phi_{0} \\
0
\end{array}\right), \text { and } \vec{\Phi}_{0}^{+}\left(\begin{array}{c}
0 \\
\Phi_{0}
\end{array}\right) \text { for } k=0 .
$$

We then define $\eta_{k}^{ \pm}=\left(\left(\eta, \vec{\Phi}_{k}^{ \pm}\right)\right)$for $k \in \mathbb{N} \cup\{0\}$ in $1 \mathrm{D}$.

Theorem 4. Assume $d=1$ and $\sigma>1$. Then the process $\tilde{\eta}$ defined by $(2.7)$ and $\tilde{\eta}(0)=0$ verifies, as $\mu_{0}$ tends to $-\lambda_{0}$, for all $t \in[0, T]$,

$$
\begin{aligned}
& \mathbb{E}\left(\left|\tilde{\eta}_{2}^{ \pm}(t)\right|^{2}\right)=\frac{\sqrt{\pi}}{4}(\sigma+1)^{\frac{1}{2 \sigma}}\left(\mu_{0}+\lambda_{0}\right)^{1 / \sigma} t+O\left(\left(\mu_{0}+\lambda_{0}\right)^{\kappa+1 / \sigma} t\right), \\
& \mathbb{E}\left(\left|\tilde{\eta}_{k}^{ \pm}(t)\right|^{2}\right)=O\left(\left(\mu_{0}+\lambda_{0}\right)^{\kappa+1 / \sigma} t\right) \quad \text { for } k \neq 2 .
\end{aligned}
$$

with $\kappa=\min \{1-1 / \sigma, 1 / 2 \sigma\}$.

Remark 2.3. Theorem 4 says that on a time scale of order $\left(\mu_{0}+\lambda_{0}\right)^{-1 / \sigma}$, one can expect that the power distribution of $\eta$ concentrates in the $\vec{\Phi}_{2}^{ \pm}$-mode in 1D. However the total energy, $\mathbb{E}\left(|\tilde{\eta}(t)|_{L^{2}}^{2}\right)$, is not conserved in (2.7), our result is different from the energy diffusion discussed in $[18]$.

Remark 2.4. The spatial dimension $d$ is limited to $d=1$. This is only because we do not have any result on the local existence of solutions for $\sigma>1$, with an existence time that only depends on the energy norm, in higher dimension. The problem is related to the lack of dispersive estimate for the linear part of (1.2) (see [8]). However, with such a result in hand, a result similar to Theorem 4 would be valid in 2D (see Remark 6.3 in Section 6).

\section{MOdULATION AND ESTIMATES ON THE EXIT TIME}

In this section, we give a proof of the existence of modulation parameters and the estimate on the exit time (2.4). The arguments are similar to those in [7] but we repeat them for the sake of completeness. The following lemma gives the evolution of the charge $Q$ and of the energy $H$ by (1.5). For the proof, refer to Theorem 3 (i) in [8].

Lemma 3.1. Assume $d=1$ and $0<\sigma$, or $d=2$ and $1 / 2 \leq \sigma \leq 1$. Let $\mu_{0} \in\left(-\lambda_{0}, \mu^{*}\right)$ be fixed. Let $u^{\varepsilon}$ be the solution of (1.5) given by Theorem 1, with $u^{\varepsilon}(0, x)=\phi_{\mu_{0}}$. Then for any stopping time $\tau<\tau^{*}\left(\phi_{\mu_{0}}\right)$ we have

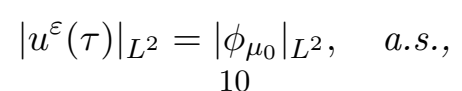




$$
H\left(u^{\varepsilon}(\tau)\right)=H\left(\phi_{\mu_{0}}\right)-2 \varepsilon \operatorname{Im} \int_{0}^{\tau} \int_{\mathbb{R}^{d}} \nabla u^{\varepsilon} \cdot x \bar{u}^{\varepsilon} d x d W(s)+2 \varepsilon^{2} \int_{0}^{\tau}\left|x u^{\varepsilon}\right|_{L^{2}}^{2} d s, \quad \text { a.s. }
$$

We give a proof of existence of the modulation parameters using the implicit function theorem under the orthogonality conditions (2.5) and (2.6). We will change parameters in subsection 6.3.

Proof of Theorem 2. Let $B_{\phi_{\mu_{0}}}(2 \alpha)=\left\{v \in \Sigma,\left|v-\phi_{\mu_{0}}\right|_{\Sigma} \leq 2 \alpha\right\}$ for $\alpha$ with $0<\alpha<\mu_{0} / 4$. Let also for some $\delta>0$,

$$
U_{\phi_{\mu_{0}}}(\delta)=\left\{v \in \Sigma, \inf _{\theta \in \mathbb{R}}\left|v-e^{i \theta} \phi_{\mu_{0}}\right|_{\Sigma}<\delta\right\} .
$$

We then consider a $C^{2}$ mapping

$$
\begin{array}{cc}
\mathcal{I}:\left(\mu_{0}-2 \alpha, \mu_{0}+2 \alpha\right) \times(-2 \alpha, 2 \alpha) \times B_{\phi_{\mu_{0}}}(2 \alpha) & \rightarrow \mathbb{R} \times \mathbb{R} \\
(\mu, \theta, u) & \mapsto\left(\mathcal{I}_{1}, \mathcal{I}_{2}\right)
\end{array}
$$

defined by

and

$$
\mathcal{I}_{1}(\mu, \theta, u)=\operatorname{Re} \int_{\mathbb{R}^{d}}\left(e^{-i \theta} u-\phi_{\mu}\right) \phi_{\mu_{0}} d x
$$

$$
\mathcal{I}_{2}(\mu, \theta, u)=\operatorname{Im} \int_{\mathbb{R}^{d}}\left(e^{-i \theta} u-\phi_{\mu}\right) \phi_{\mu_{0}} d x .
$$

We then obtain, using Proposition 1 (i) and (iii),

$$
\begin{aligned}
& \mathcal{I}\left(\mu_{0}, 0, \phi_{\mu_{0}}\right)=0, \quad \partial_{\theta} \mathcal{I}_{1}\left(\mu_{0}, 0, \phi_{\mu_{0}}\right) \partial_{\mu} \mathcal{I}_{2}\left(\mu_{0}, 0, \phi_{\mu_{0}}\right)=0, \\
& \partial_{\mu} \mathcal{I}_{1}\left(\mu_{0}, 0, \phi_{\mu_{0}}\right)=-\left.\frac{1}{2} \partial_{\mu}\left|\phi_{\mu}\right|_{L^{2}}^{2}\right|_{\mu=\mu_{0}}<0, \\
& \partial_{\theta} \mathcal{I}_{2}\left(\mu_{0}, 0, \phi_{\mu_{0}}\right)=\left|\phi_{\mu_{0}}\right|_{L^{2}}^{2}>0 .
\end{aligned}
$$

Here we apply the implicit function theorem and, for $\alpha \leq \alpha_{0}$ where $\alpha_{0}$ is sufficiently small, there exists a $C^{2}$ mapping $(\mu(u), \theta(u))$ defined for $u \in B_{\phi_{\mu_{0}}}(2 \alpha)$, such that

$$
\mathcal{I}_{1}(\mu(u), \theta(u), u)=\mathcal{I}_{2}(\mu(u), \theta(u), u)=0 .
$$

We apply this with $u=u^{\varepsilon}(t)$, we get the existence of $\mu^{\varepsilon}(t)=\mu\left(u^{\varepsilon}(t)\right)$ and $\theta^{\varepsilon}(t)=\theta\left(u^{\varepsilon}(t)\right)$ such that the orthogonality conditions (2.5) and (2.6) hold with $\varepsilon \eta^{\varepsilon}(t)=e^{-i \theta^{\varepsilon}(t)} u^{\varepsilon}(t)-\phi_{\mu^{\varepsilon}(t)}$. Since $u^{\varepsilon}(t)$ is a $\Sigma$-valued process, it follows that $u^{\varepsilon}(t)$ is a semi-martingale process in $\Sigma^{-4}$. Noting that $\mathcal{I}$ is a $C^{2}$ functional of $u$ on $\Sigma^{-4}$ (see Proposition 1 (i)), the processes $\mu^{\varepsilon}$ and $\theta^{\varepsilon}$ are given locally by a deterministic $C^{2}$ function of $u^{\varepsilon} \in \Sigma$. Then the Itô formula shows that $\mu^{\varepsilon}$ and $\theta^{\varepsilon}$ are semi-martingale processes. Moreover, since it is clear that $\mathcal{I}\left(\mu^{\varepsilon}(t), 0, e^{-i \theta^{\varepsilon}(t)} u^{\varepsilon}(t)\right)=0$, the existence of $\mu^{\varepsilon}$ and $\theta^{\varepsilon}$ holds as long as

$$
\left|\mu^{\varepsilon}(t)-\mu_{0}\right|<\alpha, \quad \text { and } \quad\left|e^{-i \theta^{\varepsilon}(t)} u^{\varepsilon}(t)-\phi_{\mu_{0}}\right|_{\Sigma}<\alpha .
$$

We now define two stopping times

$$
\begin{gathered}
\check{\tau}_{\alpha}^{\varepsilon}=\inf \left\{t \geq 0,\left|\mu^{\varepsilon}(t)-\mu_{0}\right| \geq \alpha, \quad \text { or } \quad\left|e^{-i \theta^{\varepsilon}(t)} u^{\varepsilon}(t)-\phi_{\mu_{0}}\right|_{\Sigma} \geq \alpha\right\}, \\
\tau_{\beta}^{\varepsilon}=\inf \left\{t \geq 0,\left|\mu^{\varepsilon}(t)-\mu_{0}\right| \geq \beta, \quad \text { or } \quad\left|e^{-i \theta^{\varepsilon}(t)} u^{\varepsilon}(t)-\phi_{\mu^{\varepsilon}(t)}\right|_{\Sigma} \geq \beta\right\} .
\end{gathered}
$$


The inequality $\left|\phi_{\mu_{0}}-\phi_{\mu^{\varepsilon}(t)}\right|_{\Sigma} \leq C \alpha$ holds as long as $\left|\mu^{\varepsilon}(t)-\mu_{0}\right| \leq \alpha \leq \alpha_{0}$, with a constant depending only on $\alpha_{0}$ and $\mu_{0}$. Indeed, we have

$$
\left|\phi_{\mu^{\varepsilon}(t)}-\phi_{\mu_{0}}\right|_{\Sigma} \leq\left|\mu^{\varepsilon}(t)-\mu_{0}\right| \sup _{t \leq T \wedge \tau^{\varepsilon}}\left|\partial_{\mu}\left(\phi_{\mu^{\varepsilon}(t)}\right)\right|_{\Sigma} \leq C\left(\alpha_{0}, \mu_{0}\right)\left|\mu^{\varepsilon}(t)-\mu_{0}\right| .
$$

It then follows that

$$
\tau_{\alpha}^{\varepsilon} \leq \check{\tau}_{(C+1) \alpha}^{\varepsilon} \leq \tau_{(C+1)^{2} \alpha}^{\varepsilon} .
$$

Taking $\alpha_{0}$ sufficiently small again, the processes $\theta^{\varepsilon}(t)$ and $\mu^{\varepsilon}(t)$ are defined for all $t \leq \tau_{\alpha_{0}}^{\varepsilon}$, and satisfy (2.2) and (2.3) for all $t \leq \tau_{\alpha}^{\varepsilon}, \alpha \leq \alpha_{0}$ under the orthogonality conditions (2.5) and (2.6).

It remains to prove (2.4). We give a proof in a similar way to the method in $[6,9]$. We may write a.s. for $t \leq \tau_{\alpha}^{\varepsilon}, \alpha \leq \alpha_{0}$,

$$
\begin{aligned}
& S_{\mu_{0}}\left(e^{-i \theta^{\varepsilon}(t)} u^{\varepsilon}(t, \cdot)\right)-S_{\mu_{0}}\left(\phi_{\mu^{\varepsilon}(t)}\right) \\
& =\left\langle S_{\mu_{0}}^{\prime}\left(\phi_{\mu^{\varepsilon}(t)}\right), \varepsilon \eta^{\varepsilon}(t)\right\rangle+\left\langle S_{\mu_{0}}^{\prime \prime}\left(\phi_{\mu^{\varepsilon}(t)}\right) \varepsilon \eta^{\varepsilon}(t), \varepsilon \eta^{\varepsilon}(t)\right\rangle+o\left(\left|\varepsilon \eta^{\varepsilon}(t)\right|_{\Sigma}^{2}\right) .
\end{aligned}
$$

Note that $o\left(\left|\varepsilon \eta^{\varepsilon}(t)\right|_{\Sigma}^{2}\right)$ is uniform in $\omega, \varepsilon$ and $t$, since $S_{\mu_{0}}^{\prime}\left(\phi_{\mu_{0}}\right)$ and $S_{\mu_{0}}^{\prime \prime}\left(\phi_{\mu_{0}}\right)$ depend continuously on $\mu_{0}$, and since $\left|\mu^{\varepsilon}(t)-\mu_{0}\right| \leq \alpha$ and $\left|e^{-i \theta^{\varepsilon}(t)} u^{\varepsilon}(t, \cdot)-\phi_{\mu^{\varepsilon}(t)}\right|_{\Sigma}=\left|\varepsilon \eta^{\varepsilon}(t, \cdot)\right|_{\Sigma} \leq \alpha$ for all $t \leq \tau_{\alpha}^{\varepsilon}$. We then assume $\alpha_{0}$ small enough so that the last term is less than $\frac{\nu}{2}\left|\varepsilon \eta^{\varepsilon}(t)\right|_{\Sigma}^{2}$ for all $t \leq \tau_{\alpha}^{\varepsilon}$. Since, by Proposition 1 , for any $\mu_{0} \in\left(-\lambda_{0}, \mu_{*}\right)$,

$$
\left\langle S_{\mu_{0}}^{\prime \prime}\left(\phi_{\mu_{0}}\right) \varepsilon \eta^{\varepsilon}, \varepsilon \eta^{\varepsilon}\right\rangle \geq \nu\left|\varepsilon \eta^{\varepsilon}\right|_{\Sigma}^{2}
$$

holds a.s. for $t \leq \tau_{\alpha}^{\varepsilon}$, and by the following inequality with $\sigma \geq 1 / 2$,

$$
\left|\phi_{\mu^{\varepsilon}(t)}^{2 \sigma}-\phi_{\mu_{0}}^{2 \sigma}\right|_{\Sigma} \leq\left|\mu^{\varepsilon}(t)-\mu_{0}\right| \sup _{t \leq T \wedge \tau^{\varepsilon}}\left|\partial_{\mu}\left(\phi_{\mu^{\varepsilon}(t)}^{2 \sigma}\right)\right|_{\Sigma} \leq C\left(\alpha_{0}, \mu_{0}\right)\left|\mu^{\varepsilon}(t)-\mu_{0}\right|
$$

we get

It thus follows

$$
\left\|S_{\mu_{0}}^{\prime \prime}\left(\phi_{\mu^{\varepsilon}(t)}\right)-S_{\mu_{0}}^{\prime \prime}\left(\phi_{\mu_{0}}\right)\right\|_{\mathcal{L}\left(\Sigma ; \Sigma^{-1}\right)} \leq C\left|\mu^{\varepsilon}(t)-\mu_{0}\right| .
$$

$$
\begin{aligned}
& S_{\mu_{0}}\left(e^{-i \theta^{\varepsilon}(t)} u^{\varepsilon}(t, x)\right)-S_{\mu_{0}}\left(\phi_{\mu^{\varepsilon}(t)}\right) \\
& \geq\left\langle S_{\mu_{0}}^{\prime}\left(\phi_{\mu^{\varepsilon}(t)}\right), \varepsilon \eta^{\varepsilon}(t)\right\rangle+\nu\left|\varepsilon \eta^{\varepsilon}\right|_{\Sigma}^{2}-C\left|\mu^{\varepsilon}(t)-\mu_{0}\right|\left|\varepsilon \eta^{\varepsilon}\right|_{\Sigma}^{2}-\frac{\nu}{2}\left|\varepsilon \eta^{\varepsilon}(t)\right|_{\Sigma}^{2} .
\end{aligned}
$$

On the other hand, since $S_{\mu_{0}}^{\prime}\left(\phi_{\mu_{0}}\right)=0$, using again (3.3) and (3.4), we get a.s. for $t \leq \tau_{\alpha}^{\varepsilon}$,

$$
\begin{aligned}
& \left\langle S_{\mu_{0}}^{\prime}\left(\phi_{\mu^{\varepsilon}(t)}\right), \varepsilon \eta^{\varepsilon}(t)\right\rangle=\left\langle S_{\mu_{0}}^{\prime}\left(\phi_{\mu^{\varepsilon}(t)}\right)-S_{\mu_{0}}^{\prime}\left(\phi_{\mu_{0}}\right), \varepsilon \eta^{\varepsilon}(t)\right\rangle, \\
& \left|\left\langle S_{\mu_{0}}^{\prime}\left(\phi_{\mu^{\varepsilon}(t)}\right), \varepsilon \eta^{\varepsilon}(t)\right\rangle\right| \leq C\left|\mu^{\varepsilon}(t)-\mu_{0}\right|\left|\varepsilon \eta^{\varepsilon}(t)\right|_{\Sigma} \leq \frac{\nu}{4}\left|\varepsilon \eta^{\varepsilon}(t)\right|_{\Sigma}^{2}+C\left|\mu^{\varepsilon}(t)-\mu_{0}\right|^{2} .
\end{aligned}
$$

Finally, for all $\alpha \leq \alpha_{0}$, and for all $t \leq \tau_{\alpha}^{\varepsilon}$, we obtain a.s.

$$
S_{\mu_{0}}\left(e^{-i \theta^{\varepsilon}(t)} u^{\varepsilon}(t, \cdot)\right)-S_{\mu_{0}}\left(\phi_{\mu^{\varepsilon}(t)}\right) \geq \frac{\nu}{8}\left|\varepsilon \eta^{\varepsilon}(t)\right|_{\Sigma}^{2}-C\left|\mu^{\varepsilon}(t)-\mu_{0}\right|^{2}
$$

for a constant $C$ depending only on $\alpha_{0}, \mu_{0}$ and $\nu$.

Here we estimate $\left|\mu^{\varepsilon}(t)-\mu_{0}\right|^{2}$. Let $t>0$, we denote the stopping time by $\tau=\tau_{\alpha}^{\varepsilon} \wedge t$. ¿From (3.1) and $\left(\eta^{\varepsilon}, \phi_{\mu_{0}}\right)=0$,

$$
\begin{aligned}
\left|u^{\varepsilon}(\tau)\right|_{L^{2}}^{2}=\left|\phi_{\mu_{0}}\right|_{L^{2}}^{2} & =\left|e^{i \theta^{\varepsilon}(t)}\left(\phi_{\mu^{\varepsilon}(\tau)}+\varepsilon \eta^{\varepsilon}\right)\right|_{L^{2}}^{2} \\
& =\left|\phi_{\mu^{\varepsilon}(\tau)}\right|_{L^{2}}^{2}+\left|\varepsilon \eta^{\varepsilon}\right|_{L^{2}}^{2}+2\left(\phi_{\mu^{\varepsilon}(\tau)}-\phi_{\mu_{0}}, \varepsilon \eta^{\varepsilon}\right) .
\end{aligned}
$$


Using this equality and (iii) of Proposition 1 , we have for some constants $C>0$ and $\delta>0$,

$$
\begin{aligned}
\delta\left|\mu^{\varepsilon}(t)-\mu_{0}\right| & \leq\left.|| \phi_{\mu^{\varepsilon}(\tau)}\right|_{L^{2}} ^{2}-\left|\phi_{\mu_{0}}\right|_{L^{2}}^{2} \mid \\
& \leq\left|\varepsilon \eta^{\varepsilon}\right|_{L^{2}}^{2}+2 \alpha\left|\phi_{\mu^{\varepsilon}(\tau)}-\phi_{\mu_{0}}\right|_{L^{2}} \\
& \leq\left|\varepsilon \eta^{\varepsilon}\right|_{L^{2}}^{2}+\alpha C\left(\mu_{0}\right)\left|\mu^{\varepsilon}(t)-\mu_{0}\right| .
\end{aligned}
$$

Choosing then $\alpha_{0}$ sufficiently small, we get

$$
\left|\mu^{\varepsilon}(t)-\mu_{0}\right| \leq C\left(\alpha_{0}, \mu_{0}\right)\left|\varepsilon \eta^{\varepsilon}\right|_{L^{2}}^{2}
$$

On the other hand, using $S_{\mu_{0}}^{\prime}\left(\phi_{\mu_{0}}\right)=0$ and (3.3),

$$
\left|S_{\mu_{0}}\left(\phi_{\mu^{\varepsilon}(t)}\right)-S_{\mu_{0}}\left(\phi_{\mu_{0}}\right)\right| \leq C\left(\alpha_{0}, \mu_{0}\right)\left|\mu^{\varepsilon}(t)-\mu_{0}\right|^{2} ;
$$

using the above inequality, together with (3.6), and inserting these in (3.5), we obtain

$$
\frac{\nu}{8}\left|\varepsilon \eta^{\varepsilon}(t)\right|_{\Sigma}^{2} \leq S_{\mu_{0}}\left(u^{\varepsilon}(t, \cdot)\right)-S_{\mu_{0}}\left(\phi_{\mu_{0}}\right)+C\left(\alpha_{0}, \mu_{0}\right)\left|\varepsilon \eta^{\varepsilon}\right|_{L^{2}}^{4}
$$

Again we choose $\alpha_{0}$ sufficiently small, then make use of (3.2) to get, for any $\tau \leq \tau_{\alpha}^{\varepsilon}$

$$
\begin{aligned}
\frac{\nu}{16}\left|\varepsilon \eta^{\varepsilon}(\tau)\right|_{\Sigma}^{2} & \leq S_{\mu_{0}}\left(u^{\varepsilon}(\tau, x)\right)-S_{\mu_{0}}\left(\phi_{\mu_{0}}\right)=H\left(u^{\varepsilon}(\tau, x)\right)-H\left(\phi_{\mu_{0}}\right) \\
& =-2 \varepsilon \operatorname{Im} \int_{0}^{\tau} \int_{\mathbb{R}^{d}} \nabla u^{\varepsilon}(s, x) \cdot x \bar{u}^{\varepsilon}(s, x) d x d W(s)+2 \varepsilon^{2} \int_{0}^{\tau}\left|x u^{\varepsilon}(s, \cdot)\right|_{L^{2}}^{2} d s
\end{aligned}
$$

Let us now fix $T>0$. We may write setting $\tau=\tau_{\alpha}^{\varepsilon} \wedge T$,

$$
\mathbb{P}\left(\tau_{\alpha}^{\varepsilon} \leq T\right) \leq \mathbb{P}\left(\left|\mu^{\varepsilon}(\tau)-\mu_{0}\right| \geq \alpha\right)+\mathbb{P}\left(\left|\varepsilon \eta^{\varepsilon}(\tau)\right|_{\Sigma} \geq \alpha\right) .
$$

Note that if $\left|\mu^{\varepsilon}(\tau)-\mu_{0}\right| \geq \alpha$ then $\left|\mu^{\varepsilon}(\tau)-\mu_{0}\right|=\alpha$ and $\left|\varepsilon \eta^{\varepsilon}(\tau)\right|_{\Sigma} \leq \alpha$, in particular, $\left|\varepsilon \eta^{\varepsilon}(\tau)\right|_{L^{2}} \leq$ $\alpha$. On the other hand, it follows from (3.6) that $\alpha \leq C\left(\alpha_{0}, \mu_{0}\right) \alpha^{2}$ which is impossible for $\alpha$ sufficiently small. Then $\left|\mu^{\varepsilon}(\tau)-\mu_{0}\right|<\alpha$ and

$$
\mathbb{P}\left(\tau_{\alpha}^{\varepsilon} \leq T\right) \leq \mathbb{P}\left(\left|\varepsilon \eta^{\varepsilon}(\tau)\right|_{\Sigma} \geq \alpha\right) .
$$

Now we will estimate $\mathbb{P}\left(\left|\varepsilon \eta^{\varepsilon}(\tau)\right|_{\Sigma} \geq \alpha\right)$ as in [9]. Here we remark that there exists a constant $C\left(\alpha_{0}, \mu_{0}\right)>0$ such that $\left|u^{\varepsilon}(s, \cdot)\right|_{\Sigma}^{2} \leq C$ for any $s \in[0, \tau]$. Taking $\varepsilon$ sufficiently small, depending on $C, \alpha, T$, and $\nu$ we obtain using (3.7):

$$
\begin{aligned}
\mathbb{P}\left(\left|\varepsilon \eta^{\varepsilon}(\tau)\right|_{\Sigma} \geq \alpha\right) & \leq \mathbb{P}\left(\frac{64}{\nu} \varepsilon\left|\int_{0}^{\tau} \int_{\mathbb{R}^{d}} \nabla u^{\varepsilon}(s, x) \cdot x \bar{u}^{\varepsilon}(s, x) d x d W(s)\right| \geq \frac{\alpha}{2}\right) \\
& \leq \mathbb{P}\left(\frac{64}{\nu} \varepsilon \sup _{t \in[0, T]}\left|\int_{0}^{t \wedge \tau}\left(\int_{\mathbb{R}^{d}} \nabla u^{\varepsilon}(s, x) \cdot x \bar{u}^{\varepsilon}(s, x) d x\right) d W(s)\right| \geq \frac{\alpha}{2}\right) .
\end{aligned}
$$

We conclude thanks to the classical exponential tail estimates for 1D stochastic integrals, once we have noticed that for any $t \in[0, \tau]$,

$$
\left|\int_{\mathbb{R}^{d}} \nabla u^{\varepsilon} \cdot x \bar{u}^{\varepsilon}(t, x) d x\right|^{2} \leq \sup _{t \in[0, \tau]}\left|u^{\varepsilon}(t)\right|_{\Sigma}^{2} \leq C\left(\alpha_{0}, \mu_{0}\right), \quad \text { a.s. }
$$

Remark 3.1. The stopping time $\tau_{\alpha}^{\varepsilon}$ here is the first time for which the solution quits a neighborhood of the modulated standing wave, but we do not know whether it also corresponds to the exit time of a tubular neighborhood $U_{\phi_{\mu_{0}}}(\delta)$. 
We summarize the conclusion of this section; the solution $u^{\varepsilon}(t, x)$ of Eq.(1.5) with $u^{\varepsilon}(0, x)=$ $\phi_{\mu_{0}}(x)$ may be written, for any $t \leq \tau_{\alpha}^{\varepsilon} \wedge T$, as the form (2.1) under the conditions (2.5) and (2.6), where $\theta^{\varepsilon}(t)$ and $\mu^{\varepsilon}(t)$ are semi-martingale processes and $\tau_{\alpha}^{\varepsilon}$ is a stopping time satisfying (2.4) with (2.2) and (2.3).

\section{MODULATION EQUATIONS}

In this section we derive the system of equations coupling the modulation parameters $\mu^{\varepsilon}, \theta^{\varepsilon}$, to the remaining term $\eta^{\varepsilon}$. We fix $\alpha$ so that the conclusion of Section 3 holds and we write $\tau^{\varepsilon}$ for $\tau_{\alpha}^{\varepsilon}$ from now on. Since $\mu^{\varepsilon}$ and $\theta^{\varepsilon}$ are semi-martingale processes, adapted to the filtration $\left(\mathcal{F}_{t}\right)_{t \geq 0}$ generated by $(W(t))_{t \geq 0}$, we may thus write a priori the equations for $\mu^{\varepsilon}$ and $\theta^{\varepsilon}$ in the form

$$
\left\{\begin{array}{l}
d \theta^{\varepsilon}=\mu^{\varepsilon} d t+\varepsilon y^{\varepsilon} d t+\varepsilon z^{\varepsilon} d W \\
d \mu^{\varepsilon}=\varepsilon a^{\varepsilon} d t+\varepsilon b^{\varepsilon} d W
\end{array}\right.
$$

where $a^{\varepsilon}$ and $y^{\varepsilon}$ are real valued adapted processes with paths in $L^{1}\left(0, \tau^{\varepsilon}\right)$ a.s., $z^{\varepsilon}$ and $b^{\varepsilon}$ are real valued predictable processes, with paths in $L^{2}\left(0, \tau^{\varepsilon}\right)$ a.s.

Lemma 4.1. Let $\eta^{\varepsilon}=\eta_{R}^{\varepsilon}+i \eta_{I}^{\varepsilon}$, where $\eta_{R}^{\varepsilon}=\operatorname{Re} \eta^{\varepsilon}$ and $\eta_{I}^{\varepsilon}=\operatorname{Im} \eta^{\varepsilon}$. With the above notations, $\eta_{R}^{\varepsilon}$ and $\eta_{I}^{\varepsilon}$ satisfy the equations

$$
\begin{gathered}
d \eta_{R}^{\varepsilon}=L_{\mu_{0}}^{+} \eta_{I}^{\varepsilon} d t-a^{\varepsilon} \partial_{\mu} \phi_{\mu^{\varepsilon}} d t+\left(\mu^{\varepsilon}-\mu_{0}\right) \eta_{I}^{\varepsilon} d t+\left(\phi_{\mu_{0}}^{2 \sigma}-\phi_{\mu^{\varepsilon}}^{2 \sigma}\right) \eta_{I}^{\varepsilon} d t+\varepsilon y^{\varepsilon} \eta_{I}^{\varepsilon} d t-\frac{\varepsilon}{2}|x|^{4} \phi_{\mu^{\varepsilon}} d t \\
-\frac{\varepsilon}{2}\left(z^{\varepsilon}\right)^{2} \phi_{\mu^{\varepsilon}} d t-\varepsilon z^{\varepsilon}|x|^{2} \phi_{\mu^{\varepsilon}} d t-\frac{\varepsilon}{2}\left(b^{\varepsilon}\right)^{2} \partial_{\mu}^{2} \phi_{\mu^{\varepsilon}} d t-\varepsilon h_{I}^{\varepsilon} d t-\frac{\varepsilon^{2}}{2}|x|^{4} \eta_{R}^{\varepsilon} d t \\
-\varepsilon^{2} z^{\varepsilon}|x|^{2} \eta_{R}^{\varepsilon} d t-\frac{\varepsilon^{2}}{2}\left(z^{\varepsilon}\right)^{2} \eta_{R}^{\varepsilon} d t-b^{\varepsilon} \partial_{\mu} \phi_{\mu^{\varepsilon}} d W+\varepsilon|x|^{2} \eta_{I}^{\varepsilon} d W+\varepsilon z^{\varepsilon} \eta_{I}^{\varepsilon} d W \\
d \eta_{I}^{\varepsilon}=-L_{\mu_{0}}^{-} \eta_{R}^{\varepsilon} d t-y^{\varepsilon} \phi_{\mu^{\varepsilon}} d t-\left(\mu^{\varepsilon}-\mu_{0}\right) \eta_{R}^{\varepsilon} d t+(2 \sigma+1)\left(\phi_{\mu^{\varepsilon}}^{2 \sigma}-\phi_{\mu_{0}}^{2 \sigma}\right) \eta_{R}^{\varepsilon} d t \\
-\varepsilon y^{\varepsilon} \eta_{R}^{\varepsilon} d t+\varepsilon h_{R}^{\varepsilon} d t-\frac{\varepsilon^{2}}{2}|x|^{4} \eta_{I}^{\varepsilon} d t-\varepsilon^{2} z^{\varepsilon}|x|^{2} \eta_{I}^{\varepsilon} d t-\frac{\varepsilon^{2}}{2}\left(z^{\varepsilon}\right)^{2} \eta_{I}^{\varepsilon} d t \\
-|x|^{2} \phi_{\mu^{\varepsilon}} d W-z^{\varepsilon} \phi_{\mu^{\varepsilon}} d W-\varepsilon|x|^{2} \eta_{R}^{\varepsilon} d W-\varepsilon z^{\varepsilon} \eta_{R}^{\varepsilon} d W
\end{gathered}
$$

where $h_{R}^{\varepsilon}$ and $h_{I}^{\varepsilon}$ are defined by

$$
\varepsilon^{2} h_{R}^{\varepsilon}+i \varepsilon^{2} h_{I}^{\varepsilon}=\int_{0}^{1}(1-s) \frac{\partial^{2}}{\partial s^{2}}\left(\left|\phi_{\mu^{\varepsilon}}+s \varepsilon \eta^{\varepsilon}\right|^{2 \sigma}\left(\phi_{\mu^{\varepsilon}}+s \varepsilon \eta^{\varepsilon}\right)\right) d s .
$$

Proof. First we formally derive Eqs. (4.2) and (4.3). Using the fact that $u^{\varepsilon}$ satisfies Eq.(1.5) and $\theta^{\varepsilon}$ satisfies equation (4.1), Itô formula gives

$$
\begin{aligned}
d\left(e^{-i \theta^{\varepsilon}(t)} u^{\varepsilon}(t)\right) & =e^{-i \theta^{\varepsilon}(t)}\left(i \Delta u^{\varepsilon}-i|x|^{2} u^{\varepsilon}-\frac{\varepsilon^{2}}{2}|x|^{4} u^{\varepsilon}\right. \\
& \left.+i\left|u^{\varepsilon}\right|^{2 \sigma} u^{\varepsilon}-i \mu^{\varepsilon} u^{\varepsilon}-i \varepsilon y^{\varepsilon} u^{\varepsilon}-\varepsilon^{2} z^{\varepsilon}|x|^{2} u^{\varepsilon}-\frac{\varepsilon^{2}}{2}\left(z^{\varepsilon}\right)^{2} u^{\varepsilon}\right) d t \\
& -i e^{-i \theta^{\varepsilon}(t)}\left(\varepsilon z^{\varepsilon} u^{\varepsilon}+\varepsilon|x|^{2} u^{\varepsilon}\right) d W
\end{aligned}
$$


We use Itô formula for $\phi_{\mu^{\varepsilon}(t)}$ and we get

$$
d\left(\phi_{\mu^{\varepsilon}(t)}\right)=\varepsilon a^{\varepsilon} \partial_{\mu} \phi_{\mu^{\varepsilon}(t)} d t+\frac{\varepsilon^{2}}{2}\left(b^{\varepsilon}\right)^{2} \partial_{\mu}^{2} \phi_{\mu^{\varepsilon}(t)} d t+\varepsilon b^{\varepsilon} \partial_{\mu} \phi_{\mu^{\varepsilon}(t)} d W
$$

Next, we use the following properties

$$
\begin{gathered}
L_{\mu^{\varepsilon}}^{-} \eta_{R}^{\varepsilon}=L_{\mu_{0}}^{-} \eta_{R}^{\varepsilon}+\left(\mu^{\varepsilon}-\mu_{0}\right) \eta_{R}^{\varepsilon}-(2 \sigma+1)\left(\phi_{\mu^{\varepsilon}}^{2 \sigma}-\phi_{\mu_{0}}^{2 \sigma}\right) \eta_{R}^{\varepsilon}, \\
L_{\mu^{\varepsilon}}^{+} \eta_{I}^{\varepsilon}=L_{\mu_{0}}^{+} \eta_{I}^{\varepsilon}+\left(\mu^{\varepsilon}-\mu_{0}\right) \eta_{I}^{\varepsilon}-\left(\phi_{\mu^{\varepsilon}}^{2 \sigma}-\phi_{\mu_{0}}^{2 \sigma}\right) \eta_{I}^{\varepsilon}, \\
\left(\eta_{R}^{\varepsilon}, \phi_{\mu_{0}}\right)=0, \quad\left(\eta_{I}^{\varepsilon}, \phi_{\mu_{0}}\right)=0 .
\end{gathered}
$$

Also, we write for $\sigma \geq 1 / 2$,

$$
\left|\phi_{\mu^{\varepsilon}}+\varepsilon \eta^{\varepsilon}\right|^{2 \sigma}\left(\phi_{\mu^{\varepsilon}}+\varepsilon \eta^{\varepsilon}\right)=\phi_{\mu^{\varepsilon}}^{2 \sigma+1}+\varepsilon(2 \sigma+1) \eta_{R}^{\varepsilon} \phi_{\mu^{\varepsilon}}^{2 \sigma}+i \varepsilon \eta_{I}^{\varepsilon} \phi_{\mu^{\varepsilon}}^{2 \sigma}+\varepsilon^{2} h_{R}^{\varepsilon}+i \varepsilon^{2} h_{I}^{\varepsilon} .
$$

Using these facts, (1.8) and (4.5), replacing $e^{-i \theta^{\varepsilon}(t)} u^{\varepsilon}(t)$ by $\phi_{\mu^{\varepsilon}(t)}+\varepsilon \eta^{\varepsilon}(t, x)$ in (4.4), and identifying the real and imaginary parts, we deduce the equations (4.2) and (4.3).

Here, we briefly explain how to justify the above computations; let $P_{n_{1}}$ be the projection onto the finite-dimensional space $\mathbb{R}^{n_{1}}$ spanned by the eigenfunctions $\Phi_{k}(x)$ of $-\Delta+|x|^{2}$ for $k=0,1, \cdots, n_{1}$. We use a sequence of approximations indexed by $n=\left(n_{1}, n_{2}\right) \in \mathbb{N}^{2}$. We mean, by $n$ goes to $\infty$, that first $n_{1}$ goes to $\infty$ and then $n_{2}$ goes to $\infty$. We consider the solutions $u^{\varepsilon, n}=\sum_{k=0}^{n_{1}}\left(u^{\varepsilon, n}, \Phi_{k}\right) \Phi_{k}$, of the following equation.

$d u^{\varepsilon, n}=\left(i \Delta u^{\varepsilon, n}-i|x|^{2} u^{\varepsilon, n}+\frac{\varepsilon^{2}}{2} P_{n_{1}} \Theta_{n_{2}}^{2}(x)|x|^{4} u^{\varepsilon, n}+i P_{n_{1}} g_{n_{2}}\left(u^{\varepsilon, n}\right)\right) d t-i \varepsilon P_{n_{1}} \Theta_{n_{2}}(x)|x|^{2} u^{\varepsilon, n} d W$,

where $\Theta(x)$ is a smooth function such that

$$
\Theta(x)=\left\{\begin{array}{ll}
1, & |x| \leq 1 / 2, \\
0, & |x| \geq 1
\end{array} \text { and } \quad \Theta_{n_{2}}(x)=\Theta\left(\frac{|x|^{2}}{n_{2}}\right) . \quad \text { Also, } \quad g_{n_{2}}(x)= \begin{cases}|s|^{2 \sigma} s, & |s| \leq n_{2} \\
n_{2}^{2 \sigma} s, & |s| \geq n_{2}\end{cases}\right.
$$

Since this finite system involves only globally Lipschitz functions, we see that (4.6) has a unique solution $u^{\varepsilon, n}$ with paths a.s. in $C\left(\mathbb{R}^{+}, \Sigma^{m}\right)$ with $u^{\varepsilon, n}(0)=\phi_{\mu_{0}}$ and $m \geq 1$. Moreover it can be proved similarly to [8] that $u^{\varepsilon, n}$ converges to the solution $u^{\varepsilon}$ of (1.5), in probability, in $C\left(\left[0, \tau_{\alpha}^{\varepsilon} \wedge T\right], \Sigma\right)$ as $n$ goes to $\infty$. Indeed, this convergence holds in $C\left(\left[0, \tau^{*}\left(\phi_{\mu_{0}}\right)\right), \Sigma\right)$ and it is clear that $\tau_{\alpha}^{\varepsilon}<\tau^{*}\left(\phi_{\mu_{0}}\right)$ almost surely. All the arguments in Section 3 are valid uniformly in $n$ if $n \geq n_{0}\left(\alpha_{0}\right)$ for some $n_{0}>0$. Hence, for fixed $n$, we apply the above arguments to $u^{\varepsilon, n}$ instead of $u^{\varepsilon}$ and take the limit as $n$ goes to infinity.

As in $[6,7]$, we now take the $L^{2}$ inner product of Eqs.(4.2) and (4.3) with $\phi_{\mu_{0}}$ and make use of the orthogonality conditions (2.5) and (2.6), we obtain the equations for the modulation parameters $y^{\varepsilon}, z^{\varepsilon}, a^{\varepsilon}$ and $b^{\varepsilon}$ from the identification of drift parts and that of martingale parts.

Let

$$
Z^{\varepsilon}(t)\left(\begin{array}{c}
z^{\varepsilon}(t) \\
b^{\varepsilon}(t)
\end{array}\right) \quad \text { and } \quad Y^{\varepsilon}(t)\left(\begin{array}{c}
y^{\varepsilon}(t) \\
a^{\varepsilon}(t)
\end{array}\right)
$$

Lemma 4.2. Under the assumptions of Theorem 2, the modulation parameters satisfy the system of the equations, for any $t \leq \tau^{\varepsilon}$,

and

$$
A^{\varepsilon}(t) Z^{\varepsilon}(t)=F^{\varepsilon}(t)
$$

$$
A^{\varepsilon}(t) Y^{\varepsilon}(t)=G^{\varepsilon}(t)
$$


where $Z^{\varepsilon}$ and $Y^{\varepsilon}$ are defined above, $A^{\varepsilon}$ is defined by

$$
A^{\varepsilon}(t)\left(\begin{array}{cc}
\left(\phi_{\mu^{\varepsilon}}, \phi_{\mu_{0}}\right) & 0 \\
0 & \left(\partial_{\mu} \phi_{\mu^{\varepsilon}}, \phi_{\mu_{0}}\right)
\end{array}\right)
$$

and $F^{\varepsilon}$ and $G^{\varepsilon}$ are given as follows;

$$
F^{\varepsilon}(t)\left(\begin{array}{c}
-\left(|x|^{2} \phi_{\mu^{\varepsilon}}, \phi_{\mu_{0}}\right)-\varepsilon\left(|x|^{2} \eta_{R}^{\varepsilon}, \phi_{\mu_{0}}\right) \\
\varepsilon\left(|x|^{2} \eta_{I}^{\varepsilon}, \phi_{\mu_{0}}\right)
\end{array}\right)
$$

and

$$
G^{\varepsilon}\left(\begin{array}{l}
G_{1}^{\varepsilon}(t) \\
G_{2}^{\varepsilon}(t)
\end{array}\right)
$$

with

$$
\begin{aligned}
G_{1}^{\varepsilon}(t)= & 2 \sigma\left(\eta_{R}^{\varepsilon}, \phi_{\mu_{0}}^{2 \sigma+1}\right)+(2 \sigma+1)\left(\left(\phi_{\mu^{\varepsilon}}^{2 \sigma}-\phi_{\mu_{0}}^{2 \sigma}\right) \eta_{R}^{\varepsilon}, \phi_{\mu_{0}}\right)+\varepsilon\left(h_{R}^{\varepsilon}, \phi_{\mu_{0}}\right) \\
& -\frac{1}{2} \varepsilon^{2}\left(|x|^{4} \eta_{I}^{\varepsilon}, \phi_{\mu_{0}}\right)-\varepsilon^{2} z^{\varepsilon}\left(|x|^{2} \eta_{I}^{\varepsilon}, \phi_{\mu_{0}}\right)
\end{aligned}
$$

and

$$
\begin{aligned}
G_{2}^{\varepsilon}(t)= & \left(\left(\phi_{\mu_{0}}^{2 \sigma}-\phi_{\mu^{\varepsilon}}^{2 \sigma}\right) \phi_{\mu_{0}}, \eta_{I}^{\varepsilon}\right)-\frac{\varepsilon}{2}\left(|x|^{4} \phi_{\mu^{\varepsilon}}, \phi_{\mu_{0}}\right)-\varepsilon z^{\varepsilon}\left(|x|^{2} \phi_{\mu^{\varepsilon}}, \phi_{\mu_{0}}\right)-\frac{\varepsilon}{2}\left(z^{\varepsilon}\right)^{2}\left(\phi_{\mu^{\varepsilon}}, \phi_{\mu_{0}}\right) \\
& -\frac{\varepsilon}{2}\left(b^{\varepsilon}\right)^{2}\left(\partial_{\mu}^{2} \phi_{\mu^{\varepsilon}}, \phi_{\mu_{0}}\right)-\varepsilon\left(h_{I}^{\varepsilon}, \phi_{\mu_{0}}\right)-\frac{\varepsilon^{2}}{2}\left(|x|^{4} \eta_{R}^{\varepsilon}, \phi_{\mu_{0}}\right)-\varepsilon^{2} z^{\varepsilon}\left(|x|^{2} \eta_{R}^{\varepsilon}, \phi_{\mu_{0}}\right) .
\end{aligned}
$$

We deduce from the modulation equations obtained in Lemma 4.2 the following estimates for the modulation parameters.

Corollary 4.3. Under the assumptions of Theorem 2, there is a $\alpha_{1}>0$ such that for any $\alpha \leq \alpha_{1}$, there is a constant $C\left(\mu_{0}, \alpha\right)$ with

$$
\left|z^{\varepsilon}(t)\right|+\left|b^{\varepsilon}(t)\right| \leq C\left(\mu_{0}, \alpha\right), \quad \text { for all } \quad t \leq \tau^{\varepsilon}, \quad \varepsilon \leq \varepsilon_{0} .
$$

Moreover, there are constants $C_{1}$ and $C_{2}$ depending only on $\alpha$ and $\mu_{0}$ such that

$$
\left|a^{\varepsilon}(t)\right|+\left|y^{\varepsilon}(t)\right| \leq C_{1}\left|\eta^{\varepsilon}(t)\right|_{L^{2}}+\varepsilon C_{2} \quad \text { a.s. for all } \quad t \leq \tau^{\varepsilon}, \quad \varepsilon \leq \varepsilon_{0} .
$$

In order to prove Corollary 4.3, we recall how $h_{R}^{\varepsilon}$ and $h_{I}^{\varepsilon}$ express in terms of $\phi_{\mu^{\varepsilon}}$ and $\varepsilon \eta^{\varepsilon}$ : 


$$
\begin{aligned}
h_{R}^{\varepsilon}= & \frac{2 \sigma}{\varepsilon^{2}} \int_{0}^{1}(1-s)\left[\left(\phi_{\mu^{\varepsilon}}+s \varepsilon \eta_{R}^{\varepsilon}\right)^{2}+\left(s \varepsilon \eta_{I}^{\varepsilon}\right)^{2}\right]^{\sigma-1} \\
& \times\left\{2\left(\left(\phi_{\mu^{\varepsilon}}+s \varepsilon \eta_{R}^{\varepsilon}\right) \varepsilon \eta_{R}^{\varepsilon}+s\left(\varepsilon \eta_{I}^{\varepsilon}\right)^{2}\right) \varepsilon \eta_{R}^{\varepsilon}+\left(\left(\varepsilon \eta_{R}^{\varepsilon}\right)^{2}+\left(\varepsilon \eta_{I}^{\varepsilon}\right)^{2}\right)\left(\phi_{\mu^{\varepsilon}}+s \varepsilon \eta_{R}^{\varepsilon}\right)\right\} d s \\
& +\frac{4 \sigma(\sigma-1)}{\varepsilon^{2}} \int_{0}^{1}(1-s)\left[\left(\phi_{\mu^{\varepsilon}}+s \varepsilon \eta_{R}^{\varepsilon}\right)^{2}+\left(s \varepsilon \eta_{I}^{\varepsilon}\right)^{2}\right]^{\sigma-2} \\
& \times\left\{\left(\phi_{\mu^{\varepsilon}}+s \varepsilon \eta_{R}^{\varepsilon}\right) \varepsilon \eta_{R}^{\varepsilon}+s\left(\varepsilon \eta_{I}^{\varepsilon}\right)^{2}\right\}^{2}\left(\phi_{\mu^{\varepsilon}}+s \varepsilon \eta_{R}^{\varepsilon}\right) d s \\
h_{I}^{\varepsilon}= & \frac{2 \sigma}{\varepsilon^{2}} \int_{0}^{1}(1-s)\left[\left(\phi_{\mu^{\varepsilon}}+s \varepsilon \eta_{R}^{\varepsilon}\right)^{2}+\left(s \varepsilon \eta_{I}^{\varepsilon}\right)^{2}\right]^{\sigma-1} \\
& \times\left\{2\left(\left(\phi_{\mu^{\varepsilon}}+s \varepsilon \eta_{R}^{\varepsilon}\right) \varepsilon \eta_{R}^{\varepsilon}+s\left(\varepsilon \eta_{I}^{\varepsilon}\right)^{2}\right) \varepsilon \eta_{I}^{\varepsilon}+\left(\left(\varepsilon \eta_{R}^{\varepsilon}\right)^{2}+\left(\varepsilon \eta_{I}^{\varepsilon}\right)^{2}\right) s \varepsilon \eta_{I}^{\varepsilon}\right\} d s \\
& +\frac{4 \sigma(\sigma-1)}{\varepsilon^{2}} \int_{0}^{1}(1-s)\left[\left(\phi_{\mu^{\varepsilon}}+s \varepsilon \eta_{R}^{\varepsilon}\right)^{2}+\left(s \varepsilon \eta_{I}^{\varepsilon}\right)^{2}\right]^{\sigma-2} \\
& \times\left\{\left(\phi_{\mu^{\varepsilon}}+s \varepsilon \eta_{R}^{\varepsilon}\right) \varepsilon \eta_{R}^{\varepsilon}+s\left(\varepsilon \eta_{I}^{\varepsilon}\right)^{2}\right\}^{2} s \varepsilon \eta_{I}^{\varepsilon} d s .
\end{aligned}
$$

Proof. We may write almost surely for $t \leq \tau^{\varepsilon}$ that $A^{\varepsilon}(t)=A_{0}+O\left(\left|\mu^{\varepsilon}-\mu_{0}\right|+\left|\varepsilon \eta^{\varepsilon}\right|_{\Sigma}\right)$, where

$$
A_{0}\left(\begin{array}{cc}
\left|\phi_{\mu_{0}}\right|_{L^{2}}^{2} & 0 \\
0 & \left(\partial_{\mu} \phi_{\mu_{0}}, \phi_{\mu_{0}}\right)
\end{array}\right)
$$

and $O\left(\left|\mu^{\varepsilon}-\mu_{0}\right|+\left|\varepsilon \eta^{\varepsilon}\right|_{\Sigma}\right)$ holds uniformly in $\varepsilon, t$ and $\omega$ as long as $t \leq \tau^{\varepsilon}$. Hence, choosing $\alpha \leq \alpha_{1}$ smaller if necessary (depending only on $\mu_{0}$ ), it follows that setting

$$
\tilde{A}^{\varepsilon}(t)=A_{0}+\mathbb{1}_{\left[0, \tau^{\varepsilon}\right)}(t)\left(A^{\varepsilon}(t)-A_{0}\right),
$$

the matrix $\tilde{A}^{\varepsilon}(t)$ is invertible, for all $t$, and for a.e. $\omega \in \Omega$,

$$
\left\|\left(\tilde{A}^{\varepsilon}(t)\right)^{-1}\right\|_{\mathcal{L}\left(\mathbb{R}^{2}\right)} \leq C\left(\mu_{0}, \alpha\right) .
$$

Then Eq. (4.8) may be solved as $Z^{\varepsilon}(t)=\left(\tilde{A}^{\varepsilon}(t)\right)^{-1} F^{\varepsilon}(t)$ for $t \leq \tau^{\varepsilon}$, which implies, using (3.3),

$$
\left|z^{\varepsilon}(t)\right|+\left|b^{\varepsilon}(t)\right| \leq C\left(\mu_{0}, \alpha\right)\left|F^{\varepsilon}(t)\right| \leq C\left(\mu_{0}, \alpha\right)\left(\left|\phi_{\mu_{0}}\right|_{\Sigma}^{2}+\left|\mu^{\varepsilon}-\mu_{0}\right|^{2}+\left|\varepsilon \eta^{\varepsilon}\right|_{\Sigma}^{2}\right),
$$

for any $t \leq \tau^{\varepsilon}$.

We now prove the estimates for the drift part. Thanks to the Sobolev embedding $\Sigma \subset L^{4 \sigma}$ if $d=1,2$, we have

$$
\begin{aligned}
\left|\left(\varepsilon h_{R}^{\varepsilon}, \phi_{\mu_{0}}\right)\right|+\left|\left(\varepsilon h_{I}^{\varepsilon}, \phi_{\mu_{0}}\right)\right| & \leq \varepsilon \int_{0}^{1}(1-s)\left(\left(\left|\phi_{\mu^{\varepsilon}}\right|^{2 \sigma}+\left|\varepsilon \eta^{\varepsilon}\right|^{2 \sigma}\right)\left(\left|\eta_{R}^{\varepsilon}\right|+\left|\eta_{I}^{\varepsilon}\right|\right), \phi_{\mu_{0}}\right) d s \\
& \leq C\left(\left|\phi_{\mu^{\varepsilon}}\right|_{L^{4 \sigma}}^{2 \sigma}+\left|\varepsilon \eta^{\varepsilon}\right|_{L^{4 \sigma}}^{2 \sigma}\right)\left|\eta^{\varepsilon}\right|_{L^{2}}\left|\phi_{\mu_{0}}\right|_{L^{\infty}} \\
& \leq C\left(\alpha, \mu_{0}\right)\left|\eta^{\varepsilon}\right|_{L^{2}} .
\end{aligned}
$$

The estimates (4.15), (3.4) and (4.13) lead to

$$
\left|G_{1}^{\varepsilon}(t)\right|+\left|G_{2}^{\varepsilon}(t)\right| \leq C_{1}\left|\eta^{\varepsilon}(t)\right|_{L^{2}}+\varepsilon C_{2}, \quad \text { a.s. for all } t \leq \tau^{\varepsilon},
$$


where $C_{1}$ and $C_{2}$ depend only on $\mu_{0}$ and $\alpha$. Lastly applying the same argument with $\left(\tilde{A}^{\varepsilon}(t)\right)^{-1}$ as above, (4.14) follows, with possibly different constants.

We state the following corollary which will be useful to prove Lemmas 5.1, 5.2 and 5.4 below.

Corollary 4.4. Under the assumptions of Theorem 2, there exist some constants $C_{1}$ and $C_{2}^{\prime}$ such that

$$
\left|\mu^{\varepsilon}(t)-\mu_{0}\right| \leq \varepsilon\left(C_{1} \int_{0}^{T \wedge \tau^{\varepsilon}}\left|\eta^{\varepsilon}(s)\right|_{L^{2}} d s+T C_{2}^{\prime}\right), \quad \text { a.s. for all } \quad t \leq T \wedge \tau^{\varepsilon} .
$$

Proof. This corollary follows immediately from Corollary 4.3 and

$$
\mu^{\varepsilon}\left(t \wedge \tau^{\varepsilon}\right)-\mu_{0}=\varepsilon \int_{0}^{t \wedge \tau^{\varepsilon}} a^{\varepsilon}(s) d s+\varepsilon \int_{0}^{t \wedge \tau^{\varepsilon}} b^{\varepsilon}(s) d W .
$$

\section{ESTIMATES ON THE REMAINDER TERM AND CONVERGENCE}

Let $\eta=\eta_{R}+i \eta_{I}$ with $\eta_{R}=\operatorname{Re} \eta$ and $\eta_{I}=\operatorname{Im} \eta$. Consider the equation

$$
d \eta=J \mathcal{L}_{\mu_{0}} d t-y\left(\begin{array}{c}
0 \\
\phi_{\mu_{0}}
\end{array}\right) d t-\left(\begin{array}{c}
0 \\
|x|^{2} \phi_{\mu_{0}}
\end{array}\right) d W-z\left(\begin{array}{c}
0 \\
\phi_{\mu_{0}}
\end{array}\right) d W
$$

where we set for all $t \geq 0$,

$$
y(t)=-\frac{\left(L_{\mu_{0}}^{-} \eta_{R}, \phi_{\mu_{0}}\right)}{\left|\phi_{\mu_{0}}\right|_{L^{2}}^{2}}, \quad z(t)=-\frac{\left|x \phi_{\mu_{0}}\right|_{L^{2}}^{2}}{\left|\phi_{\mu_{0}}\right|_{L^{2}}^{2}} .
$$

Note that $J \mathcal{L}_{-\lambda_{0}}$ is skew-adjoint and generates a $C_{0}$ unitary group by Stone's theorem. Other terms are linear, or deterministic and bounded in $\Sigma$, thus, equation (5.1) with $\eta(0)=0$ has a unique adapted solution $\eta \in C\left(\mathbb{R}^{+}, \Sigma\right)$, a.s. Moreover, it is easy to check that $\eta$ satisfies $\left(\eta_{R}, \phi_{\mu_{0}}\right)=0$ and $\left(\eta_{I}, \phi_{\mu_{0}}\right)=0$. In this section we will show that $\eta^{\varepsilon}$ converges to $\eta$ in probability in $C\left(\left[0, \tau^{\varepsilon} \wedge T\right], L^{2}\right)$ for any $T>0$ as $\varepsilon$ goes to 0 . First we list some estimates to prove that convergence. For the proof of these estimates, see Section 7. We note that $\eta^{\varepsilon}, y^{\varepsilon}, z^{\varepsilon}, a^{\varepsilon}, b^{\varepsilon}$ given by Section 3 and (4.1) are a priori defined only for $t \leq \tau^{\varepsilon}$. We define them for $t \in \mathbb{R}^{+}$by simply setting $\eta^{\varepsilon}(t)=\eta^{\varepsilon}\left(\tau^{\varepsilon}\right)$ for $t \geq \tau^{\varepsilon}$ and the same for the others.

Lemma 5.1. Let $T>0$ fixed. Under the assumption of Theorem 2, there exist constants $C_{1}$ and $C_{2}$ depending only on $T, \alpha, \mu_{0}$, ( and $N$ if $d=2$ ) such that

$$
\text { (i) } \mathbb{E}\left(\sup _{t \leq \tilde{\tau}^{\varepsilon} \wedge T}\left|\eta^{\varepsilon}(t)\right|_{L^{2}}^{2}\right) \leq C_{1} \text {, and (ii) } \mathbb{E}\left(\sup _{t \leq \tilde{\tau}^{\varepsilon} \wedge T}\left|\eta^{\varepsilon}(t)\right|_{L^{2}}^{4}\right) \leq C_{2} \text {, }
$$

where $\tilde{\tau}^{\varepsilon}=\tau^{\varepsilon}$ if $d=1$, or $\tilde{\tau}^{\varepsilon}=\tau^{\varepsilon} \wedge \bar{\tau}_{N}^{\varepsilon}$ if $d=2$, with

$$
\bar{\tau}_{N}^{\varepsilon}=\inf \left\{t \leq \tau^{\varepsilon} \wedge T,\left|\varepsilon \eta^{\varepsilon}\right|_{\Sigma^{2}} \geq N\right\}, \quad \text { for any } N>0 .
$$


Lemma 5.2. Let $T>0$ fixed. Under the assumption of Theorem 3, there exists a constant $C_{3}$ depending only on $T, \alpha, \mu_{0}$, (and $N$ if $d=2$ ) such that

$$
\mathbb{E}\left(\sup _{t \leq \tilde{\tau}^{\varepsilon} \wedge T}\left|\eta^{\varepsilon}(t)\right|_{\Sigma}^{4}\right) \leq C_{3}
$$

where $\tilde{\tau}^{\varepsilon}$ is defined in Lemma 5.1.

Lemma 5.3. Let $T>0$ fixed. Under the assumption of Theorem 2, there exist $C_{4}, C_{5}$ and $C_{6}$ depending only on $T, \alpha, \mu_{0}$ such that

$$
\text { (i) } \mathbb{E}\left(\sup _{t \leq T}|\eta(t)|_{L^{2}}^{4}\right) \leq C_{4}, \quad \text { (ii) } \mathbb{E}\left(\sup _{t \leq T}|\eta(t)|_{\Sigma}^{2}\right) \leq C_{5},
$$

and

$$
\text { (iii) } \mathbb{E}\left(\sup _{t \leq T}\left|\left(1+|x|^{4}\right) \eta(t)\right|_{L^{2}}^{2}\right) \leq C_{6} \text {. }
$$

We remark that the assumption $\sigma \geq 1$ is needed only for Lemma 5.2. Using these lemmas we obtain the following convergence.

Lemma 5.4. Let $T>0$ and $N>0$ be fixed. Under the assumptions of Theorem 3, $\eta^{\varepsilon}$ converges to $\eta$, as $\varepsilon$ tends to 0 , in $L^{2}\left(\Omega ; C\left(\left[0, \tilde{\tau}^{\varepsilon} \wedge T\right], L^{2}\right)\right)$.

The convergence in probability in the time interval $\left[0, \tau^{\varepsilon} \wedge T\right]$ follows from Lemma 5.4 :

Corollary 5.5. Let $T>0$ be fixed. Under the assumptions of Theorem 3, $\eta^{\varepsilon}$ converges to $\eta$, as $\varepsilon$ tends to 0 , in probability, in $C\left(\left[0, \tau^{\varepsilon} \wedge T\right], L^{2}\right)$.

First, we admit Lemma 5.4 and we prove Corollary 5.5.

Proof of Corollary 5.5. In the 1D case, the conclusion follows directly from Lemma 5.4. In $2 \mathrm{D}$, we prove that for any $\beta>0, \delta>0$

$$
\mathbb{P}\left(\sup _{t \in[0, T]}\left|\mathbb{1}_{\left[0, \tau^{\varepsilon} \wedge T\right]} \eta^{\varepsilon}-\mathbb{1}_{[0, T]} \eta\right|_{L^{2}}>\delta\right) \leq \beta
$$

provided that $\varepsilon$ is sufficiently small. We note that

$$
\begin{aligned}
\mathbb{P}\left(\sup _{t \in[0, T]}\left|\mathbb{1}_{\left[0, \tau^{\varepsilon} \wedge T\right]} \eta^{\varepsilon}-\mathbb{1}_{[0, T]} \eta\right|_{L^{2}}>\delta\right) & \leq \mathbb{P}\left(\sup _{t \in[0, T]}\left|\mathbb{1}_{\left[0, \tau^{\varepsilon} \wedge T\right]}\left(\eta^{\varepsilon}-\eta\right)\right|_{L^{2}}>\delta\right) \\
& +\mathbb{P}\left(\tau^{\varepsilon} \wedge T<T\right) .
\end{aligned}
$$

It follows from (2.4) that for any $\beta>0$ there exists $\varepsilon_{0}>0, \mathbb{P}\left(\tau^{\varepsilon} \wedge T<T\right) \leq \beta / 3$ for any $\varepsilon \leq \varepsilon_{0}$. On the other hand,

$$
\begin{aligned}
\mathbb{P}\left(\sup _{t \in[0, T]}\left|\mathbb{1}_{\left[0, \tau^{\varepsilon} \wedge T\right]}\left(\eta^{\varepsilon}-\eta\right)\right|_{L^{2}}>\delta\right) & \leq \mathbb{P}\left(\sup _{t \in[0, T]}\left|\mathbb{1}_{\left[0, \bar{\tau}_{N}^{\varepsilon} \wedge \tau^{\varepsilon} \wedge T\right]}\left(\eta^{\varepsilon}-\eta\right)\right|_{L^{2}}>\delta\right) \\
& +\mathbb{P}\left(\bar{\tau}_{N}^{\varepsilon} \wedge \tau^{\varepsilon} \wedge T<T\right) .
\end{aligned}
$$

Concerning the second term, we first show that for any $\beta>0$ there exist $N_{0}$ and $\varepsilon_{0}$ such that for any $\varepsilon \leq \varepsilon_{0}$,

$$
\mathbb{P}\left(\sup _{t \in\left[0, \tau^{\varepsilon} \wedge T\right]}\left|\varepsilon \eta^{\varepsilon}\right|_{\Sigma^{2}} \geq N_{0}\right) \leq \beta / 3
$$


Remarking $\varepsilon \eta^{\varepsilon}(t, x)=e^{-i \theta^{\varepsilon}(t)} u^{\varepsilon}(t, x)-\phi_{\mu^{\varepsilon}(t)}$, it suffices to mention the estimate

$\mathbb{P}\left(\sup _{t \in\left[0, \tau^{\varepsilon} \wedge T\right]}\left|u^{\varepsilon}(t)\right|_{\Sigma^{2}} \geq N\right) \leq \frac{\mathbb{E}\left(\sup _{t \in\left[\tau^{\varepsilon} \wedge T\right]}\left(1+\log \left(1+\left|u^{\varepsilon}(t)\right|_{\Sigma^{2}}^{2}\right)\right)\right)}{1+\log \left(1+N^{2}\right)} \leq \frac{C\left(T, \mu_{0}, \alpha\right)}{1+\log \left(1+N^{2}\right)} \leq \frac{\beta}{3}$

for sufficiently large $N$; this follows simply from the bound (2.10) of Lemma 2.7 in [8]. It shows

$$
\mathbb{P}\left(\bar{\tau}_{N_{0}}^{\varepsilon} \wedge \tau^{\varepsilon} \wedge T<T\right) \leq \mathbb{P}\left(\sup _{t \in\left[0, \tau^{\varepsilon} \wedge T\right]}\left|\varepsilon \eta^{\varepsilon}\right|_{\Sigma^{2}} \geq N_{0}\right) \leq \beta / 3
$$

Now, using Lemma 5.4, we have for any $\beta>0$,

$$
\mathbb{P}\left(\sup _{t \in[0, T]}\left|\mathbb{1}_{\left[0, \bar{\tau}_{N_{0}}^{\varepsilon} \wedge \tau^{\varepsilon} \wedge T\right]}\left(\eta^{\varepsilon}-\eta\right)\right|_{L^{2}}>\delta\right) \leq \frac{\beta}{3},
$$

provided $\varepsilon$ is sufficiently small, and we get (5.3) by simply fixing $N=N_{0}$ in (5.4).

Next, we prove Lemma 5.4.

Proof of Lemma 5.4. Let $v^{\varepsilon}=\eta^{\varepsilon}-\eta$, and also let the imaginary part and real part of $v^{\varepsilon}$ be $v_{R}^{\varepsilon}=\eta_{R}^{\varepsilon}-\eta_{R}, v_{I}^{\varepsilon}=\eta_{I}^{\varepsilon}-\eta_{I}$ respectively. Then, $v_{R}^{\varepsilon}$ and $v_{I}^{\varepsilon}$ satisfy for $t \leq \tau^{\varepsilon} \wedge T$ :

$$
\begin{aligned}
d v_{R}^{\varepsilon}= & L_{\mu_{0}}^{+} v_{I}^{\varepsilon} d t-a^{\varepsilon} \partial_{\mu} \phi_{\mu^{\varepsilon}} d t+\left(\mu^{\varepsilon}-\mu_{0}\right) \eta_{I}^{\varepsilon} d t+\left(\phi_{\mu_{0}}^{2 \sigma}-\phi_{\mu^{\varepsilon}}^{2 \sigma}\right) \eta_{I}^{\varepsilon} d t-\frac{\varepsilon}{2}|x|^{4} \phi_{\mu^{\varepsilon}} d t \\
& -\varepsilon z^{\varepsilon}|x|^{2} \phi_{\mu^{\varepsilon}} d t+\varepsilon y^{\varepsilon} \eta_{I}^{\varepsilon} d t-\varepsilon h_{I}^{\varepsilon} d t-\frac{\varepsilon}{2}\left(b^{\varepsilon}\right)^{2} \partial_{\mu}^{2} \phi_{\mu^{\varepsilon}} d t-\frac{\varepsilon}{2}\left(z^{\varepsilon}\right)^{2} \phi_{\mu^{\varepsilon}} d t \\
& -\frac{\varepsilon^{2}}{2}|x|^{4} \eta_{R}^{\varepsilon} d t-\varepsilon^{2} z^{\varepsilon}|x|^{2} \eta_{R}^{\varepsilon} d t-\frac{\varepsilon^{2}}{2}\left(z^{\varepsilon}\right)^{2} \eta_{R}^{\varepsilon} d t-b^{\varepsilon} \partial_{\mu} \phi_{\mu^{\varepsilon}} d W+\varepsilon|x|^{2} \eta_{I}^{\varepsilon} d W+\varepsilon z^{\varepsilon} \eta_{I}^{\varepsilon} d W, \\
d v_{I}^{\varepsilon}= & -L_{\mu_{0}}^{-} v_{R}^{\varepsilon} d t-y^{\varepsilon} \phi_{\mu^{\varepsilon}} d t+y \phi_{\mu_{0}} d t-\left(\mu^{\varepsilon}-\mu_{0}\right) \eta_{R}^{\varepsilon} d t+(2 \sigma+1)\left(\phi_{\mu^{\varepsilon}}^{2 \sigma}-\phi_{\mu_{0}}^{2 \sigma}\right) \eta_{R}^{\varepsilon} d t \\
& +\varepsilon h_{R}^{\varepsilon} d t-\varepsilon y^{\varepsilon} \eta_{R}^{\varepsilon} d t-\frac{\varepsilon^{2}}{2}|x|^{4} \eta_{I}^{\varepsilon} d t-\varepsilon^{2} z^{\varepsilon}|x|^{2} \eta_{I}^{\varepsilon} d t-\frac{\varepsilon^{2}}{2}\left(z^{\varepsilon}\right)^{2} \eta_{I}^{\varepsilon} d t-|x|^{2}\left(\phi_{\mu^{\varepsilon}}-\phi_{\mu_{0}}\right) d W \\
& -z^{\varepsilon} \phi_{\mu^{\varepsilon}} d W+z \phi_{\mu_{0}} d W-\varepsilon|x|^{2} \eta_{R}^{\varepsilon} d W-\varepsilon z^{\varepsilon} \eta_{R}^{\varepsilon} d W .
\end{aligned}
$$

Now we apply Itô formula to $L^{2}$ norms of $\left|v_{R}^{\varepsilon}\right|_{L^{2}}^{2}$ and $\left|v_{I}^{\varepsilon}\right|_{L^{2}}^{2}$, then we obtain, after some compensations, for any $\tau=\tilde{\tau}^{\varepsilon} \wedge t$,

$$
\left|v_{R}^{\varepsilon}(\tau)\right|_{L^{2}}^{2}+\left|v_{I}^{\varepsilon}(\tau)\right|_{L^{2}}^{2}=\int_{0}^{\tau}\left(D_{1}(s)+D_{2}(s)+D_{3}(s)\right) d s+\int_{0}^{\tau} M_{1}(s) d W(s)
$$


where

$$
\begin{aligned}
D_{1}(t)=\quad 4 & \sigma\left(v_{I}^{\varepsilon}, \phi_{\mu_{0}}^{2 \sigma} v_{R}^{\varepsilon}\right)-2\left(v_{I}^{\varepsilon}, y^{\varepsilon} \phi_{\mu^{\varepsilon}}-y \phi_{\mu_{0}}\right)-\varepsilon\left(v_{R}^{\varepsilon},|x|^{4} \phi_{\mu^{\varepsilon}}\right) \\
& -2 \varepsilon z^{\varepsilon}\left(v_{R}^{\varepsilon},|x|^{2} \phi_{\mu^{\varepsilon}}\right)-\varepsilon\left(b^{\varepsilon}\right)^{2}\left(v_{R}^{\varepsilon}, \partial_{\mu}^{2} \phi_{\mu^{\varepsilon}}\right)-\varepsilon\left(z^{\varepsilon}\right)^{2}\left(v_{R}^{\varepsilon}, \phi_{\mu^{\varepsilon}}\right), \\
D_{2}(t)=\left.\left.|| x\right|^{2}\left(\phi_{\mu^{\varepsilon}}-\phi_{\mu_{0}}\right)\right|_{L^{2}} ^{2}+2 z^{\varepsilon}\left(|x|^{2}\left(\phi_{\mu^{\varepsilon}}-\phi_{\mu_{0}}\right), \phi_{\mu^{\varepsilon}}\right)-2 z\left(|x|^{2}\left(\phi_{\mu^{\varepsilon}}-\phi_{\mu_{0}}\right), \phi_{\mu_{0}}\right) & -2\left(\mu^{\varepsilon}-\mu_{0}\right)\left(\eta_{R}, \eta_{I}^{\varepsilon}\right)+2\left(\mu^{\varepsilon}-\mu_{0}\right)\left(\eta_{I}, \eta_{R}^{\varepsilon}\right)-2\left(\left(\phi_{\mu_{0}}^{2 \sigma}-\phi_{\mu^{\varepsilon}}^{2 \sigma}\right) \eta_{I}^{\varepsilon}, \eta_{R}\right) \\
& -2(2 \sigma+1)\left(\eta_{I},\left(\phi_{\mu^{\varepsilon}}^{2 \sigma}-\phi_{\mu_{0}}^{2 \sigma}\right) \eta_{R}^{\varepsilon}\right)+4 \sigma\left(\eta_{I}^{\varepsilon},\left(\phi_{\mu^{\varepsilon}}^{2 \sigma}-\phi_{\mu_{0}}^{2 \sigma}\right) \eta_{R}^{\varepsilon}\right) \\
& +\left(b^{\varepsilon}\right)^{2}\left|\partial_{\mu} \phi_{\mu^{\varepsilon}}\right|_{L^{2}}^{2}+\left|z^{\varepsilon} \phi_{\mu^{\varepsilon}}-z \phi_{\mu_{0}}\right|_{L^{2}}^{2}-2 \varepsilon y^{\varepsilon}\left(\eta_{R}, \eta_{I}^{\varepsilon}\right)+2 \varepsilon y^{\varepsilon}\left(\eta_{I}, \eta_{R}^{\varepsilon}\right) \\
& -2 \varepsilon b^{\varepsilon}\left(|x|^{2} \eta_{I}^{\varepsilon}, \partial_{\mu} \phi_{\mu^{\varepsilon}}\right)-2 \varepsilon z^{\varepsilon} b^{\varepsilon}\left(\eta_{I}^{\varepsilon}, \partial_{\mu} \phi_{\mu^{\varepsilon}}\right)+2 \varepsilon\left(|x|^{2}\left(\phi_{\mu^{\varepsilon}}-\phi_{\mu_{0}}\right),|x|^{2} \eta_{R}\right) \\
& +\varepsilon z^{\varepsilon}\left(|x|^{2}\left(\phi_{\mu^{\varepsilon}}-\phi_{\mu_{0}}\right), \eta_{R}^{\varepsilon}\right)+2 \varepsilon z^{\varepsilon}\left(|x|^{2} \eta_{R}^{\varepsilon}, \phi_{\mu^{\varepsilon}}^{\varepsilon}-2 \varepsilon z\left(|x|^{2} \eta_{R}^{\varepsilon}, \phi_{\mu_{0}}\right)+2 \varepsilon\left(z^{\varepsilon}\right)^{2}\left(\phi_{\mu^{\varepsilon}}, \eta_{R}^{\varepsilon}\right)\right. \\
& -2 \varepsilon z^{\varepsilon} z\left(\eta_{R}^{\varepsilon}, \phi_{\mu_{0}}\right)+2 \varepsilon^{2} z^{\varepsilon}\left(\eta_{R},|x|^{2} \eta_{R}^{\varepsilon}\right)+\varepsilon^{2}\left(|x|^{3} \eta_{R},|x| \eta_{R}^{\varepsilon}\right)+\varepsilon^{2}\left(|x|^{3} \eta_{I},|x| \eta_{I}^{\varepsilon}\right) \\
& +\varepsilon^{2}\left(z^{\varepsilon}\right)^{2}\left(\eta_{R}, \eta_{R}^{\varepsilon}\right)+\varepsilon^{2}\left(z^{\varepsilon}\right)^{2}\left(\eta_{I}, \eta_{I}^{\varepsilon}\right)+2 \varepsilon^{2} z^{\varepsilon}\left(\eta_{I},|x|^{2} \eta_{I}^{\varepsilon}\right), \\
D_{3}(t)=\quad- & \left(h_{I}^{\varepsilon}, v_{R}^{\varepsilon}\right)+\varepsilon\left(h_{R}^{\varepsilon}, v_{I}^{\varepsilon}\right)-2 a^{\varepsilon}\left(v_{R}^{\varepsilon}, \partial_{\mu} \phi_{\mu^{\varepsilon}}\right), \\
M_{1}(t)=- & 2 \varepsilon\left(\eta_{R},|x|^{2} \eta_{I}^{\varepsilon}\right)+2 \varepsilon\left(\eta_{I},|x|^{2} \eta_{R}^{\varepsilon}\right)-2 \varepsilon z^{\varepsilon}\left(\eta_{R}, \eta_{I}^{\varepsilon}\right) \\
& +2 \varepsilon z^{\varepsilon}\left(\eta_{I}, \eta_{R}^{\varepsilon}\right)-2 b^{\varepsilon}\left(v_{R}^{\varepsilon}, \partial_{\mu} \phi_{\mu^{\varepsilon}}\right)-2\left(v_{I}^{\varepsilon},|x|^{2}\left(\phi_{\mu^{\varepsilon}}-\phi_{\mu_{0}}\right)\right)-2\left(v_{I}^{\varepsilon}, z^{\varepsilon} \phi_{\mu^{\varepsilon}}-z \phi_{\mu_{0}}\right) .
\end{aligned}
$$

Once we have obtained that, for $\varepsilon$ sufficiently small,

$$
\mathbb{E}\left(\sup _{t \leq T \wedge \tilde{\tau}^{\varepsilon}}\left|v_{R}^{\varepsilon}(t)\right|_{L^{2}}^{2}+\left|v_{I}^{\varepsilon}(t)\right|_{L^{2}}^{2}\right) \leq C(T) \int_{0}^{T} \mathbb{E}\left(\sup _{t \leq T \wedge \tilde{\tau}^{\varepsilon}}\left|v_{R}^{\varepsilon}(t)\right|_{L^{2}}^{2}+\left|v_{I}^{\varepsilon}(t)\right|_{L^{2}}^{2}\right) d t+\varepsilon C^{\prime} T,
$$

where $C^{\prime}$ may depend on $N$, we will conclude by Gronwall's lemma, that

$$
\mathbb{E}\left(\sup _{t \leq T \wedge \tilde{\tau}^{\varepsilon}}\left|v_{R}^{\varepsilon}(t)\right|_{L^{2}}^{2}+\left|v_{I}^{\varepsilon}(t)\right|_{L^{2}}^{2}\right) \leq \varepsilon C^{\prime} T^{2} \exp (C T),
$$

i.e.,

$$
\mathbb{E}\left(\sup _{t \leq T \wedge \tau^{\varepsilon}}\left|\eta^{\varepsilon}(t)-\eta(t)\right|_{L^{2}}^{2}\right) \rightarrow 0, \quad \text { as } \quad \varepsilon \rightarrow 0 .
$$

We first consider the drift part $D_{1}$. Remarking Proposition 1 (i), (v) and (4.13), we have the estimate for $t \leq \tau^{\varepsilon} \wedge T$,

$$
\left|D_{1}(t)\right| \leq C\left|v^{\varepsilon}(t)\right|_{L^{2}}^{2}+\varepsilon C^{\prime}
$$

where the constants depend only on $\mu_{0}, \sigma$ and $\alpha$ except the term $-2\left(v_{I}^{\varepsilon}, y^{\varepsilon} \phi_{\mu^{\varepsilon}}-y \phi_{\mu_{0}}\right)$. Let us explain how to majorize this term. Noting that

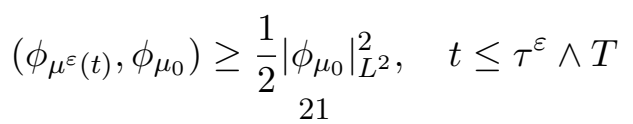


and using (4.13), (3.3) and (3.4),

$$
\begin{aligned}
\left|y^{\varepsilon}(t)-y(t)\right|= & \mid-\frac{\left(L_{\mu_{0}}^{-} v_{R}^{\varepsilon}, \phi_{\mu_{0}}\right)}{\left|\phi_{\mu_{0}}\right|_{L^{2}}^{2}}+\left(L_{\mu_{0}}^{-} \eta_{R}^{\varepsilon}, \phi_{\mu_{0}}\right)\left(\frac{1}{\left|\phi_{\mu_{0}}\right|_{L^{2}}^{2}}-\frac{1}{\left(\phi_{\mu^{\varepsilon}}, \phi_{\mu_{0}}\right)}\right) \\
& +(2 \sigma+1) \frac{1}{\left(\phi_{\mu^{\varepsilon}}, \phi_{\mu_{0}}\right)}\left(\left(\phi_{\mu^{\varepsilon}}^{2 \sigma}-\phi_{\mu_{0}}^{2 \sigma}\right) \eta_{R}^{\varepsilon}, \phi_{\mu_{0}}\right)-\frac{\varepsilon^{2}}{2\left(\phi_{\mu^{\varepsilon}}, \phi_{\mu_{0}}\right)}\left(|x|^{4} \eta_{I}^{\varepsilon}, \phi_{\mu_{0}}\right) \\
& -\frac{\varepsilon^{2} z^{\varepsilon}}{\left(\phi_{\mu^{\varepsilon}}, \phi_{\mu_{0}}\right)}\left(|x|^{2} \eta_{I}^{\varepsilon}, \phi_{\mu_{0}}\right) \mid \leq C\left(\alpha, \mu_{0}\right)\left(\left|v_{R}^{\varepsilon}\right|_{L^{2}}+\left|\mu^{\varepsilon}-\mu_{0}\right|\left|\eta_{R}^{\varepsilon}\right|_{L^{2}}+\varepsilon^{2}\left|\eta_{I}^{\varepsilon}\right|_{L^{2}}\right) .
\end{aligned}
$$

Then, we have

$$
\begin{aligned}
\left|\left(v_{I}^{\varepsilon}, y^{\varepsilon} \phi_{\mu^{\varepsilon}}-y \phi_{\mu_{0}}\right)\right| \leq & \left|\left(v_{I}^{\varepsilon}, y^{\varepsilon}\left(\phi_{\mu^{\varepsilon}}-\phi_{\mu_{0}}\right)\right)\right|+\left|\left(y^{\varepsilon}-y\right)\left(v_{I}^{\varepsilon}, \phi_{\mu_{0}}\right)\right| \\
\leq & C\left(\alpha, \mu_{0}\right)\left|\mu^{\varepsilon}-\mu_{0}\right|\left|v_{I}^{\varepsilon}\right|_{L^{2}}\left(\left|\eta^{\varepsilon}\right|_{L^{2}}+\varepsilon\right)+C\left(\alpha, \mu_{0}\right)\left|y^{\varepsilon}-y\right|\left|v_{I}^{\varepsilon}\right|_{L^{2}} \\
\leq & C\left(\alpha, \mu_{0}\right)\left(\left|v_{I}^{\varepsilon}\right|_{L^{2}}\left|v_{R}^{\varepsilon}\right|_{L^{2}}+\left.\left|\mu^{\varepsilon}-\mu_{0}\right|\left|v_{I}^{\varepsilon}\right|\right|_{L^{2}}\left|\eta^{\varepsilon}\right|_{L^{2}}\right. \\
& \left.+\varepsilon^{2}\left|v_{I}^{\varepsilon}\right|_{L^{2}}\left|\eta_{I}^{\varepsilon}\right|_{L^{2}}+\varepsilon\left|\mu^{\varepsilon}-\mu_{0}\right|\left|v_{I}^{\varepsilon}\right|_{L^{2}}\right)
\end{aligned}
$$

and it suffices to estimate, putting $\tau=\tilde{\tau}^{\varepsilon} \wedge T$,

$$
\mathbb{E} \int_{0}^{T} \sup _{t \leq \tau}\left(\left|\mu^{\varepsilon}-\mu_{0}\right|\left|v_{I}^{\varepsilon}\right|_{L^{2}}\left|\eta^{\varepsilon}\right|_{L^{2}}\right) d t
$$

Note that

$$
\begin{aligned}
& \int_{0}^{T} \mathbb{E}\left(\sup _{t \leq \tau}\left|\mu^{\varepsilon}-\mu_{0}\right|\left|v_{I}^{\varepsilon}\right|_{L^{2}}\left|\eta^{\varepsilon}\right|_{L^{2}}\right) d t \\
& \leq \int_{0}^{T} \mathbb{E}\left(\sup _{t \leq \tau}\left|\mu^{\varepsilon}-\mu_{0}\right|^{4}\right)^{1 / 4} \mathbb{E}\left(\sup _{t \leq \tau}\left|\eta^{\varepsilon}\right|_{L^{2}}^{4}\right)^{1 / 4} \mathbb{E}\left(\sup _{t \leq \tau}\left|v_{I}^{\varepsilon}\right|_{L^{2}}^{2}\right)^{1 / 2} d t \\
& \leq \varepsilon C(T)+\varepsilon C^{\prime}(T) \int_{0}^{T} \mathbb{E}\left(\sup _{t \leq \tau}\left|v_{I}^{\varepsilon}\right|_{L^{2}}^{2}\right) d t
\end{aligned}
$$

where we have used (4.16), Lemma 5.1 (ii) in the second inequality. Note that we have actually from (4.16), by Hölder inequality,

$$
\left|\mu^{\varepsilon}(t)-\mu_{0}\right| \leq \varepsilon C_{1} T^{3 / 4}\left(\int_{0}^{T} \sup _{s \leq \tau}\left|\eta^{\varepsilon}(s)\right|_{L^{2}}^{4} d s\right)^{1 / 4}+\varepsilon T C_{2}^{\prime} .
$$

Therefore,

$$
\mathbb{E}\left(\sup _{t \leq \tau}\left|\mu^{\varepsilon}(t)-\mu_{0}\right|^{4}\right)^{1 / 4} \leq \varepsilon C T^{3 / 4}\left(\mathbb{E} \int_{0}^{T} \sup _{s \leq \tau}\left|\eta^{\varepsilon}(s)\right|_{L^{2}}^{4} d s\right)^{1 / 4}+\varepsilon C T .
$$

The terms in $D_{2}$ are estimated as

$$
\mathbb{E}\left(\int_{0}^{T} \sup _{t \leq \tilde{\tau}^{\varepsilon} \wedge T}\left|D_{2}(t)\right| d t\right) \leq \varepsilon C(T) .
$$


We study some representative terms in $D_{2}$ in what follows. The first term is estimated as, using Proposition 1 (i) (v),

$$
\left.\left.|| x\right|^{2}\left(\phi_{\mu^{\varepsilon}(t)}-\phi_{\mu_{0}}\right)\right|_{L^{2}} ^{2} \leq\left.\left. C\left(\alpha, \mu_{0}\right)\left|\mu^{\varepsilon}(t)-\mu_{0}\right|^{2} \sup _{t \leq \tau^{\varepsilon} \wedge T}|| x\right|^{2} \partial_{\mu} \phi_{\mu^{\varepsilon}}\right|_{L^{2}} ^{2} \leq C\left(\alpha, \mu_{0}\right)\left|\mu^{\varepsilon}(t)-\mu_{0}\right|^{2} .
$$

Then using (4.16) together with Lemma 5.1 (i), we get

$$
\mathbb{E}\left(\int_{0}^{T} \sup _{t \leq \tilde{\tau}^{\varepsilon} \wedge T}\left(\left.\left.|| x\right|^{2}\left(\phi_{\mu^{\varepsilon}(t)}-\phi_{\mu_{0}}\right)\right|_{L^{2}} ^{2}\right) d t\right) \leq \varepsilon C\left(\alpha, \mu_{0}, T\right) .
$$

This argument is also valid for the 2 nd and the 3 rd terms. Concerning the 4 th and 5 th terms, putting $\tau=\tilde{\tau}^{\varepsilon} \wedge T$,

$$
\begin{aligned}
& \left|\int_{0}^{\tau}\left(-2\left(\mu^{\varepsilon}-\mu_{0}\right)\left(\eta_{R}, \eta_{I}^{\varepsilon}\right)+2\left(\mu^{\varepsilon}-\mu_{0}\right)\left(\eta_{I}, \eta_{R}^{\varepsilon}\right)\right) d t\right| \\
& \quad \leq C \int_{0}^{T} \sup _{t \leq \tau}\left(\left|\mu^{\varepsilon}-\mu_{0}\right||\eta|_{L^{2}}\left|\eta^{\varepsilon}\right|_{L^{2}}\right) d t
\end{aligned}
$$

which is estimated as (5.8).

For the 6th term, we estimate simply using (3.4) and Proposition 1 (i),

$$
\int_{0}^{\tau}\left|-2\left(\left(\phi_{\mu_{0}}^{2 \sigma}-\phi_{\mu^{\varepsilon}}^{2 \sigma}\right) \eta_{I}^{\varepsilon}, \eta_{R}\right)\right| d t \leq C\left(\alpha, \mu_{0}\right) \int_{0}^{\tau}\left|\mu^{\varepsilon}(t)-\mu_{0}\right|\left|\eta_{I}^{\varepsilon}(t)\right|_{L^{2}}\left|\eta_{R}(t)\right|_{L^{2}} d t
$$

and then we may continue the computation as (5.8) above. The 7 th and 8 th terms are similarly estimated.

The 9 th term is estimated as follows, writing $b^{\varepsilon}$ in details. First we note

$$
\left(\partial_{\mu} \phi_{\mu^{\varepsilon}}, \phi_{\mu_{0}}\right) \geq \frac{1}{2}\left(\partial_{\mu} \phi_{\mu_{0}}, \phi_{\mu_{0}}\right)>0, \quad \text { for } t \leq \tau^{\varepsilon} \wedge T
$$

by Proposition 1 (i), taking $\alpha$ smaller if necessary. Then, using Lemma 4.2,

$$
\left(b^{\varepsilon}\right)^{2}\left|\partial_{\mu} \phi_{\mu^{\varepsilon}}\right|_{L^{2}}^{2}=\varepsilon^{2} \frac{\left(\eta_{I}^{\varepsilon},|x|^{2} \phi_{\mu_{0}}\right)^{2}}{\left(\partial_{\mu} \phi_{\mu^{\varepsilon}}, \phi_{\mu_{0}}\right)^{2}}\left|\partial_{\mu} \phi_{\mu^{\varepsilon}}\right|_{L^{2}}^{2} \leq \varepsilon^{2} C\left(\mu_{0}, \alpha\right)\left|\eta_{I}^{\varepsilon}\right|_{L^{2}}^{2}
$$

The use of Lemma 5.1 (i) leads to (5.9).

To estimate the 10th term we remark that

$$
\left|z^{\varepsilon}(t)-z(t)\right| \leq C \varepsilon\left|\eta_{R}^{\varepsilon}\right|_{L^{2}}+C\left|\mu^{\varepsilon}-\mu_{0}\right|
$$

holds. Indeed, we can check this easily developing $z^{\varepsilon}$ in details (see Lemma 4.2 and Eq. (5.2))

$$
\begin{aligned}
z^{\varepsilon}(t)-z(t)= & -\varepsilon \frac{\left(\eta_{R}^{\varepsilon},|x|^{2} \phi_{\mu_{0}}\right)}{\left(\phi_{\mu^{\varepsilon}}, \phi_{\mu_{0}}\right)}+\frac{\left(|x|^{2} \phi_{\mu_{0}}, \phi_{\mu_{0}}\right)}{\left|\phi_{\mu_{0}}\right|_{L^{2}}^{2}}-\frac{\left(|x|^{2} \phi_{\mu^{\varepsilon}}, \phi_{\mu_{0}}\right)}{\left(\phi_{\mu^{\varepsilon}}, \phi_{\mu_{0}}\right)} \\
= & -\varepsilon \frac{\left(\eta_{R}^{\varepsilon},|x|^{2} \phi_{\mu_{0}}\right)}{\left(\phi_{\mu^{\varepsilon}}, \phi_{\mu_{0}}\right)} \\
& -\frac{\left(|x|^{2}\left(\phi_{\mu^{\varepsilon}}-\phi_{\mu_{0}}\right), \phi_{\mu_{0}}\right)}{\left(\phi_{\mu^{\varepsilon}}, \phi_{\mu_{0}}\right)}+\frac{\left(\phi_{\mu^{\varepsilon}}-\phi_{\mu_{0}}, \phi_{\mu_{0}}\right)}{\left|\phi_{\mu_{0}}\right|_{L^{2}}^{2}\left(\phi_{\mu^{\varepsilon}}, \phi_{\mu_{0}}\right)}\left(|x|^{2} \phi_{\mu_{0}}, \phi_{\mu_{0}}\right) .
\end{aligned}
$$


Recalling (5.7), then, using (4.13) and (3.3),

$$
\begin{aligned}
\int_{0}^{\tau}\left|z^{\varepsilon} \phi_{\mu^{\varepsilon}}-z \phi_{\mu_{0}}\right|_{L^{2}}^{2} d t & \leq 2 \int_{0}^{\tau}\left|z^{\varepsilon}\left(\phi_{\mu^{\varepsilon}}-\phi_{\mu_{0}}\right)\right|_{L^{2}}^{2} d t+2 \int_{0}^{\tau}\left|\left(z^{\varepsilon}-z\right) \phi_{\mu_{0}}\right|_{L^{2}}^{2} d t \\
& \leq C \int_{0}^{\tau}\left|\mu^{\varepsilon}-\mu_{0}\right|^{2} d t+C \varepsilon \int_{0}^{\tau}\left|\eta_{R}^{\varepsilon}\right|_{L^{2}}^{2} d t
\end{aligned}
$$

and

$$
\int_{0}^{T} \mathbb{E}\left(\sup _{t \leq \tau}\left|z^{\varepsilon} \phi_{\mu^{\varepsilon}}-z \phi_{\mu_{0}}\right|_{L^{2}}^{2}\right) d t \leq \varepsilon C(T),
$$

from (4.16) and Lemma 5.1 (i).

All the other terms are similarly majorized; Lemma 5.3 (iii) is required for the 21st, 22nd, 23rd and 26th terms; the verification is left to the reader.

We estimate the terms of $D_{3}$. Lemma 5.2 is needed here, together with the regularity $\sigma \geq 1$ of the nonlinearity. We first note that

$$
\begin{aligned}
\mid-\varepsilon \int_{0}^{t \wedge \tilde{\tau}^{\varepsilon}}\left(h_{I}^{\varepsilon}, v_{R}^{\varepsilon}\right) d s & +\varepsilon \int_{0}^{t \wedge \tilde{\tau}^{\varepsilon}}\left(h_{R}^{\varepsilon}, v_{I}^{\varepsilon}\right) d s \mid \\
& \leq \int_{0}^{t \wedge \tilde{\tau}^{\varepsilon}}\left(\varepsilon\left|\eta^{\varepsilon}\right|^{2}\left(\left|\phi_{\mu^{\varepsilon}}\right|^{2 \sigma-1}+\left|\varepsilon \eta^{\varepsilon}\right|^{2 \sigma-1}\right),\left|v^{\varepsilon}\right|\right) d s \\
& \leq \varepsilon C\left(\alpha, \mu_{0}\right) \int_{0}^{t \wedge \tilde{\tau}^{\varepsilon}}\left|\eta^{\varepsilon}\right|_{\Sigma}^{2}\left|v^{\varepsilon}\right|_{L^{2}} d s \\
& \leq C\left(\alpha, \mu_{0}\right) C(T)\left(\int_{0}^{t \wedge \tilde{\tau}^{\varepsilon}} \varepsilon^{2}\left|\eta^{\varepsilon}\right|_{\Sigma}^{4} d s+\int_{0}^{t \wedge \tilde{\tau}^{\varepsilon}}\left|v^{\varepsilon}\right|_{L^{2}}^{2} d s\right),
\end{aligned}
$$

where we have used the Sobolev embedding $\Sigma \subset L^{4 \sigma+2}\left(\mathbb{R}^{d}\right)$ with $d=1$ or 2 , in the second inequality. Then, an application of Lemma 5.2 implies (5.6) for the terms of $D_{3}$, with a constant $C^{\prime}$ that may depend on $N$. In order to estimate $-a^{\varepsilon}\left(v_{R}^{\varepsilon}, \partial_{\mu} \phi_{\mu^{\varepsilon}}\right)$, we look at $a^{\varepsilon}$ in details. From Lemma 4.2,

$$
\begin{aligned}
& -2 a^{\varepsilon}\left(v_{R}^{\varepsilon}, \partial_{\mu} \phi_{\mu^{\varepsilon}}\right) \\
= & -\frac{2\left(v_{R}^{\varepsilon}, \partial_{\mu} \phi_{\mu^{\varepsilon}}\right)}{\left(\partial_{\mu} \phi_{\mu^{\varepsilon}}, \phi_{\mu_{0}}\right)} \times\left[-\frac{1}{2} \varepsilon\left(|x|^{4} \phi_{\mu^{\varepsilon}}, \phi_{\mu_{0}}\right)-\varepsilon z^{\varepsilon}\left(|x|^{2} \phi_{\mu^{\varepsilon}}, \phi_{\mu_{0}}\right)-\left(\left(\phi_{\mu^{\varepsilon}}^{2 \sigma}-\phi_{\mu_{0}}^{2 \sigma}\right) \phi_{\mu_{0}}, \eta_{I}^{\varepsilon}\right)\right. \\
& \left.-\frac{\varepsilon}{2}\left(z^{\varepsilon}\right)^{2}\left(\phi_{\mu^{\varepsilon}}, \phi_{\mu_{0}}\right)-\frac{1}{2} \varepsilon^{2}\left(\eta_{R}^{\varepsilon},|x|^{4} \phi_{\mu_{0}}\right)-\varepsilon^{2} z^{\varepsilon}\left(\eta_{R}^{\varepsilon},|x|^{2} \phi_{\mu_{0}}\right)-\frac{\varepsilon}{2}\left(b^{\varepsilon}\right)^{2}\left(\partial_{\mu}^{2} \phi_{\mu^{\varepsilon}}, \phi_{\mu_{0}}\right)-\varepsilon\left(h_{I}^{\varepsilon}, \phi_{\mu_{0}}\right)\right] .
\end{aligned}
$$

Except the terms $-\varepsilon\left(h_{I}^{\varepsilon}, \phi_{\mu_{0}}\right)$ and $-\left(\left(\phi_{\mu^{\varepsilon}}^{2 \sigma}-\phi_{\mu_{0}}^{2 \sigma}\right) \phi_{\mu_{0}}, \eta_{I}^{\varepsilon}\right)$, we see easily that all the terms inside the bracket are bounded by $\varepsilon C\left(\alpha, \mu_{0}\right)$, using (4.13) and noting $\left|\varepsilon \eta^{\varepsilon}(t)\right|_{L^{2}} \leq C\left(\alpha, \mu_{0}\right)$ for $t \leq \tau^{\varepsilon} \wedge T$. On the other hand, by (3.4) and (5.12) we get

$$
\left|\left(\left(\phi_{\mu^{\varepsilon}}^{2 \sigma}-\phi_{\mu_{0}}^{2 \sigma}\right) \phi_{\mu_{0}}, \eta_{I}^{\varepsilon}\right)\right| \leq C\left(\alpha, \mu_{0}\right)\left|\mu^{\varepsilon}-\mu_{0}\right|\left|\eta_{I}^{\varepsilon}\right|_{L^{2}}, \quad\left|\varepsilon\left(h_{I}^{\varepsilon}, \phi_{\mu_{0}}\right)\right| \leq \varepsilon C\left(\alpha, \mu_{0}\right)\left|\eta^{\varepsilon}\right|_{\Sigma}^{2} .
$$

Recalling (5.10), we have

$$
\left|-2 a^{\varepsilon}\left(v_{R}^{\varepsilon}, \partial_{\mu} \phi_{\mu^{\varepsilon}}\right)\right| \leq C\left(\alpha, \mu_{0}\right)\left\{\varepsilon\left|v_{R}^{\varepsilon}\right|_{L^{2}}+\varepsilon\left|\eta^{\varepsilon}\right|_{\Sigma}^{2}\left|v_{R}^{\varepsilon}\right|_{L^{2}}+\left|\mu^{\varepsilon}-\mu_{0}\right|\left|\eta_{I}^{\varepsilon}\right|_{L^{2}}\left|v_{R}^{\varepsilon}\right|_{L^{2}}\right\},
$$

which is estimated similarly as above. 
Next, we consider the martingale part $M_{1}$. We give a proof for the terms, as representative ones, $-2\left(v_{I}^{\varepsilon},|x|^{2}\left(\phi_{\mu^{\varepsilon}}-\phi_{\mu_{0}}\right)\right)$, and $\left(v_{I}^{\varepsilon}, z^{\varepsilon} \phi_{\mu^{\varepsilon}}-z \phi_{\mu_{0}}\right)$. In a same way we can deal with the other terms of $M_{1}$. First we mention that it is possible to estimate

$$
\left|-2\left(v_{I}^{\varepsilon},|x|^{2}\left(\phi_{\mu^{\varepsilon}}-\phi_{\mu_{0}}\right)\right)\right| \leq\left.\left. C\left|v_{I}^{\varepsilon}\right|_{L^{2}}|| x\right|^{2}\left(\phi_{\mu^{\varepsilon}}-\phi_{\mu_{0}}\right)\right|_{L^{2}} \leq C\left|\mu^{\varepsilon}(t)-\mu_{0}\right|\left|v_{I}^{\varepsilon}\right|_{L^{2}},
$$

using (3.3), but with $\Sigma^{2}$ instead of $\Sigma$. With this observation, we have, putting $\tau=\tilde{\tau}^{\varepsilon} \wedge T$,

$$
\begin{aligned}
\mathbb{E}\left(\sup _{t \leq \tau}\left|\int_{0}^{t \wedge \tau}\left(v_{I}^{\varepsilon},|x|^{2}\left(\phi_{\mu^{\varepsilon}}-\phi_{\mu_{0}}\right)\right) d W\right|\right) & \leq C \mathbb{E}\left(\left(\int_{0}^{\tau}\left|v_{I}^{\varepsilon}\right|_{L^{2}}^{2}\left|\mu^{\varepsilon}-\mu_{0}\right|^{2} d s\right)^{1 / 2}\right) \\
& \leq C T^{1 / 2} \mathbb{E}\left(\sup _{t \leq \tau}\left|v_{I}^{\varepsilon}\right|_{L^{2}}\left|\mu^{\varepsilon}-\mu_{0}\right|\right) \\
& \left.\leq \varepsilon C(T) \mathbb{E}\left(\sup _{t \leq \tau}\left|v_{I}^{\varepsilon}\right|_{L^{2}}\left(\int_{0}^{T}\left|\eta^{\varepsilon}(s)\right|_{L^{2}} d s\right)+1\right)\right) \\
& \leq \varepsilon C(T) \mathbb{E}\left(\sup _{t \leq \tau}\left|v_{I}^{\varepsilon}\right|_{L^{2}}^{2}\right)+\varepsilon C(T),
\end{aligned}
$$

where we have used a martingale inequality in the first inequality, (4.16) in the third one, and Cauchy-Schwarz inequality together with Lemma 5.1 (i) in the last inequality.

Next we write $\left(v_{I}^{\varepsilon}, z^{\varepsilon} \phi_{\mu^{\varepsilon}}-z \phi_{\mu_{0}}\right)$ as follows.

$$
\int_{0}^{\tau}\left(v_{I}^{\varepsilon}, z^{\varepsilon} \phi_{\mu^{\varepsilon}}-z \phi_{\mu_{0}}\right) d W=\int_{0}^{\tau}\left(v_{I}^{\varepsilon}, z^{\varepsilon}\left(\phi_{\mu^{\varepsilon}}-\phi_{\mu_{0}}\right)\right) d W+\int_{0}^{\tau}\left(z^{\varepsilon}-z\right)\left(v_{I}^{\varepsilon}, \phi_{\mu_{0}}\right) d W .
$$

Only the second term requires an explanation, since the first term may be estimated as above. We recall the estimate (5.11), and we obtain

$$
\begin{aligned}
\mathbb{E}\left(\sup _{t \leq \tau}\left|\int_{0}^{t}\left(z^{\varepsilon}-z\right)\left(v_{I}^{\varepsilon}, \phi_{\mu_{0}}\right) d W\right|\right) \leq & C \mathbb{E}\left(\left(\int_{0}^{\tau}\left|z^{\varepsilon}-z\right|^{2}\left|v_{I}^{\varepsilon}\right|_{L^{2}}^{2}\left|\phi_{\mu_{0}}\right|_{L^{2}}^{2} d s\right)^{1 / 2}\right) \\
\leq & \varepsilon C T^{1 / 2} \mathbb{E}\left(\sup _{t \leq \tau}\left|\eta_{R}^{\varepsilon}\right|_{L^{2}}\left|v_{I}^{\varepsilon}\right|_{L^{2}}\right) \\
& +C T^{1 / 2} \mathbb{E}\left(\sup _{t \leq \tau}\left|\mu^{\varepsilon}(t)-\mu_{0}\right|\left|v_{I}^{\varepsilon}\right|_{L^{2}}\right) .
\end{aligned}
$$

Then this right hand side is clearly majorized as previously (see (5.8)). Proceeding in the same way with the other terms, we get

$$
\mathbb{E}\left(\sup _{t \leq \tau}\left|\int_{0}^{t \wedge \tau} M_{1}(s) d W(s)\right|\right) \leq \varepsilon C(T) \mathbb{E}\left(\sup _{t \leq \tau}\left|v^{\varepsilon}\right|_{L^{2}}^{2}\right)+\varepsilon C^{\prime}(T)
$$

and (5.6) holds, assuming that $\varepsilon$ is small enough so that $\varepsilon C(T) \leq 1 / 2$.

\section{THE LIMIT EQUATION}

In the previous sections we have seen that a remaining term $\eta^{\varepsilon}$ satisfying the orthogonality conditions (2.5) and (2.6) converges to a process $\eta(t)$ defined by (5.1) as $\varepsilon$ goes to zero, in 
probability, in $C\left(\left[0, \tau^{\varepsilon} \wedge T\right], L^{2}\right)$. Also, the modulation parameters verify, at order one in $\varepsilon$, the system

$$
\left\{\begin{array}{l}
d \mu^{\varepsilon}=o(\varepsilon) \\
d \theta^{\varepsilon}=\mu_{0} d t+\varepsilon y(t) d t+\varepsilon z(t) d W+o(\varepsilon),
\end{array}\right.
$$

where $y(t)$ and $z(t)$ are defined by (5.2).

In this section we study the statistical properties of this process $\eta(t)$, in particular, as mentioned in the introduction, we are interested in the quantities

$$
\mathbb{E}\left(\left|\eta_{k}^{ \pm}(t)\right|^{2}\right), \quad k \in\{0\} \cup \mathbb{N} \text {, if } d=1 .
$$

For this purpose, we proceed in the following way.

- Investigate the properties of $\phi_{\mu_{0}}$ when $\mu_{0}$ is close to $-\lambda_{0}$

- Analyze the spectral properties of the operator $J \mathcal{L}_{\mu_{0}}$

- Simplify the coupling terms in Eq.(6.1)

At the end of this section, we will give a proof of Theorem 4, deriving the master equation in our case.

6.1. Properties of $\phi_{\mu_{0}}$. First, we give some arguments and references concerning the proof of Proposition 1. Concerning (i), we refer to Theorem 18 of Shatah and Strauss [27], for the fact that $\mu \mapsto \phi_{\mu}$ is $C^{1}$ (or $C^{2}$ ) with values in $\Sigma$. To obtain the same result with $\Sigma^{2}$ (or $\Sigma^{4}$ ) instead of $\Sigma$, it suffices to differentiate Eq.(1.8) with respect to $\mu$, noting that $\phi_{\mu}$ is real valued, and then to use a bootstrap argument, inverting the operator $-\Delta+|x|^{2}+1$. See [10] for the proof of (ii) which implies (iii); indeed, assume $\left(\partial_{\mu} \phi_{\mu}, \phi_{\mu}\right)=0$ and (ii). Then we have

$$
0=-\left(\phi_{\mu}, \partial_{\mu} \phi_{\mu}\right)=\left\langle L_{\mu}^{-}\left(\partial_{\mu} \phi_{\mu}\right), \partial_{\mu} \phi_{\mu}\right\rangle \geq \nu\left|\partial_{\mu} \phi_{\mu}\right|_{\Sigma}^{2}>0
$$

which is a contradiction. Suppose that there exists $\mu_{1} \in\left(-\lambda_{0}, \mu^{*}\right)$ such that $\left(\partial_{\mu} \phi_{\mu_{1}}, \phi_{\mu_{1}}\right)<0$. Then $\psi(\mu, x)=\frac{\left|\phi_{\mu_{1}}\right|_{L^{2}}}{\left|\phi_{\mu}\right|_{L^{2}}} \phi_{\mu}(x)$ satisfies $\left(\partial_{\mu} \psi_{\mu_{1}}, \phi_{\mu_{1}}\right)=0$ and

$$
\left\langle S^{\prime \prime}\left(\phi_{\mu_{1}}\right) \partial_{\mu} \psi_{\mu_{1}}, \partial_{\mu} \psi_{\mu_{1}}\right\rangle \leq\left(\partial_{\mu} \phi_{\mu_{1}}, \phi_{\mu_{1}}\right)<0,
$$

which also contradicts to (ii). The statement of (iv) and (v) has been shown in [11].

We now put $\nu=\mu+\lambda_{0}$. Eq.(1.8) is equivalent to

$$
-\Delta \phi_{\nu}+|x|^{2} \phi_{\nu}+\left(\nu-\lambda_{0}\right) \phi_{\nu}-\phi_{\nu}^{2 \sigma+1}=0 .
$$

Lemma 6.1. Assume $\mu \in\left(-\lambda_{0}, \mu^{*}\right)$. Let $d=1,2$ and $\sigma \geq 1 / 2$. Let also $\phi_{\nu}$ be a solution of (6.2). For any $\delta \in(0,2)$, there exist $C=C(\delta)>0$ such that for $\nu$ sufficiently small,

$$
\begin{aligned}
& \text { (i) }\left|\phi_{\nu}-C_{*} \nu^{\frac{1}{2 \sigma}} \Phi_{0}\right|_{\Sigma} \leq C \nu^{\frac{2 \sigma+1}{2 \sigma}}, \quad \text { (ii) }\left.|| x\right|^{2} \phi_{\nu}-\left.C_{*} \nu^{\frac{1}{2 \sigma}}|x|^{2} \Phi_{0}\right|_{L^{2}} \leq C \nu^{\left(1-\frac{\delta}{2}\right) \frac{2 \sigma+1}{2 \sigma}}, \\
& \text { (iii) }\left|\partial_{\nu} \phi_{\nu}-\frac{1}{2 \sigma} C_{*} \nu^{\frac{1}{2 \sigma}-1} \Phi_{0}\right|_{\Sigma} \leq C \nu^{\frac{1}{2 \sigma}} .
\end{aligned}
$$

Moreover if $\sigma \geq 1$ and $d=2$,

$$
\text { (iv) }\left|\phi_{\nu}-C_{*} \nu^{\frac{1}{2 \sigma}} \Phi_{0}\right|_{\Sigma^{2}} \leq C \nu^{\frac{2 \sigma+1}{4 \sigma}} .
$$

Here we have put $C_{*}=\left|\Phi_{0}\right|_{L^{2 \sigma+2}}^{-\frac{\sigma+1}{\sigma}}=(\sigma+1)^{\frac{d}{4 \sigma}} \pi^{\frac{d}{4}}$. 
Proof. The part (i) has already been proved in [10], we recall some ingredients used in [10] which will be used for the proof of (ii) and (iii). If we decompose $\phi_{\nu}=a_{\nu} \Phi_{0}+y_{\nu}$ with $a_{\nu} \in \mathbb{R}$ and $\left(\Phi_{0}, y_{\nu}\right)_{\Sigma}=0$, then

$$
\left|\phi_{\nu}\right|_{\Sigma} \leq \nu^{\frac{1}{2 \sigma}} C_{*}, \quad\left|y_{\nu}\right|_{\Sigma} \leq C(\sigma) \nu^{\frac{2 \sigma+1}{2 \sigma}}, \quad\left|a_{\nu}\right| \leq C(\sigma) \nu^{\frac{1}{2 \sigma}} .
$$

In order to prove (ii), we also use this decomposition. Let $1 / \theta+1 / \theta^{\prime}=1$ so that, $2 \leq \theta(2-\delta)$ and $\theta^{\prime} \geq 1$. It follows from Hölder inequality and Sobolev embedding,

$$
\begin{aligned}
\left.\left.|| x\right|^{2} y_{\nu}\right|_{L^{2}} ^{2} & =\left.\left.|| x\right|^{2}\left(\phi_{\nu}-a_{\nu} \Phi_{0}\right)\right|_{L^{2}} ^{2} \\
& \leq\left.\left. C\left|\phi_{\nu}-a_{\nu} \Phi_{0}\right|_{L^{\theta(2-\delta)}}^{2-\delta}|| x\right|^{4}\left(\phi_{\nu}-a_{\nu} \Phi_{0}\right)^{\delta}\right|_{L^{\theta^{\prime}}} \\
& \leq C(\delta)\left|\phi_{\nu}-a_{\nu} \Phi_{0}\right|_{\Sigma}^{2-\delta} \leq C(\delta) \nu^{(2-\delta) \frac{2 \sigma+1}{2 \sigma}} .
\end{aligned}
$$

The quantity $\left.\left.|| x\right|^{4}\left(\phi_{\nu}-a_{\nu} \Phi_{0}\right)^{\delta}\right|_{L^{\theta^{\prime}}}$ is indeed bounded uniformly in $\nu$ from Proposition 1 (iv) (v). This shows (ii).

Next we prove (iii). In the same way, we decompose $\partial_{\nu} \phi_{\nu}=b_{\nu} \Phi_{0}+z_{\nu}$ with $b_{\nu} \in \mathbb{R}$ and $\left(\Phi_{0}, z_{\nu}\right)_{\Sigma}=0$. We insert this decomposition into the equation for $\partial_{\nu} \phi_{\nu}$, i.e.,

$$
-\Delta \partial_{\nu} \phi_{\nu}+|x|^{2} \partial_{\nu} \phi_{\nu}+\left(\nu-\lambda_{0}\right) \partial_{\nu} \phi_{\nu}-(2 \sigma+1) \phi_{\nu}^{2 \sigma} \partial_{\nu} \phi_{\nu}=-\phi_{\nu}
$$

then we have

$$
\begin{aligned}
-\Delta\left(b_{\nu} \Phi_{0}+z_{\nu}\right)+|x|^{2}\left(b_{\nu} \Phi_{0}+z_{\nu}\right)+\left(\nu-\lambda_{0}\right)\left(b_{\nu} \Phi_{0}+z_{\nu}\right) & \\
- & (2 \sigma+1)\left(a_{\nu} \Phi_{0}+y_{\nu}\right)^{2 \sigma}\left(b_{\nu} \Phi_{0}+z_{\nu}\right)=-\left(a_{\nu} \Phi_{0}+y_{\nu}\right) .
\end{aligned}
$$

Taking the $L^{2}$ product with $z_{\nu}$, we get

$$
\begin{gathered}
\left|z_{\nu}\right|_{\Sigma}^{2}+\left(\nu-\lambda_{0}\right)\left|z_{\nu}\right|_{L^{2}}^{2}-\left(y_{\nu}, z_{\nu}\right)+(2 \sigma+1)\left(\left(a_{\nu} \Phi_{0}+y_{\nu}\right)^{2 \sigma} b_{\nu} \Phi_{0}, z_{\nu}\right) \\
+(2 \sigma+1)\left(\left(a_{\nu} \Phi_{0}+y_{\nu}\right)^{2 \sigma} z_{\nu}, z_{\nu}\right) .
\end{gathered}
$$

Since $\left(\Phi_{0}, z_{\nu}\right)=\left(\Phi_{0}, z_{\nu}\right)_{\Sigma}=0, z_{\nu}$ satisfies

$$
\left(\left(-\Delta+|x|^{2}\right) z_{\nu}, z_{\nu}\right) \geq \lambda_{1}\left|z_{\nu}\right|_{L^{2}}^{2},
$$

where $\lambda_{1}$ is the second eigenvalue of $-\Delta+|x|^{2}$ and $\lambda_{1}>\lambda_{0}=d \geq 1$. Thus,

$$
\left|z_{\nu}\right|_{\Sigma}^{2} \geq\left(\lambda_{1}+1\right)\left|z_{\nu}\right|_{L^{2}}^{2}
$$

If $\nu$ is small enough, then $\nu-\lambda_{0}<0$ and so we have

$$
\left|z_{\nu}\right|_{\Sigma}^{2}-\left(\lambda_{0}-\nu\right)\left|z_{\nu}\right|_{L^{2}}^{2} \geq\left|z_{\nu}\right|_{\Sigma}^{2}-\frac{\lambda_{0}-\nu}{\lambda_{1}+1}\left|z_{\nu}\right|_{\Sigma}^{2}>\frac{1+\nu}{\lambda_{1}+1}\left|z_{\nu}\right|_{\Sigma}^{2}
$$

We also estimate the right hand side of (6.5) and we obtain, putting $C_{0}=1 /\left(\lambda_{1}+1\right)$,

$$
\begin{aligned}
C_{0}\left|z_{\nu}\right|_{\Sigma}^{2} \leq & \left|y_{\nu}\right|_{L^{2}}\left|z_{\nu}\right|_{\Sigma}+C(\sigma) C_{*}^{-\frac{\sigma(2 \sigma+1)}{\sigma+1}}\left|b_{\nu}\right|\left|a_{\nu}\right|^{2 \sigma}\left|z_{\nu}\right|_{\Sigma}+C(\sigma) C_{*}^{-\frac{\sigma}{\sigma+1}}\left|b_{\nu}\right|\left|y_{\nu}\right|_{L^{2 \sigma+2}}^{2 \sigma}\left|z_{\nu}\right|_{\Sigma} \\
& +C(\sigma) C_{*}^{-\frac{2 \sigma^{2}}{\sigma+1}}\left|a_{\nu}\right|^{2 \sigma}\left|z_{\nu}\right|_{\Sigma}^{2}+C(\sigma)\left|y_{\nu}\right|_{L^{2 \sigma+2}}^{2 \sigma}\left|z_{\nu}\right|_{\Sigma}^{2}
\end{aligned}
$$

Taking $\nu$ so small that $C_{0}-C(\sigma) C_{*}^{-\frac{2 \sigma^{2}}{\sigma+1}}\left|a_{\nu}\right|^{2 \sigma}-C(\sigma)\left|y_{\nu}\right|_{L^{2 \sigma+2}}^{2 \sigma}<1 / 2$ (see (6.3)), and using the order in $\nu$ of $a_{\nu}$ and $y_{\nu}$, we obtain

$$
\left|z_{\nu}\right|_{\Sigma} \leq C \nu^{\frac{2 \sigma+1}{2 \sigma}}+C\left(\nu+\nu^{2 \sigma+1}\right)\left|b_{\nu}\right| .
$$


On the other hand, if we take the $L^{2}$ product with $\Phi_{0}$ in Eq.(6.4),

$$
\left(\nu-(2 \sigma+1)\left(\phi_{\nu}^{2 \sigma} \Phi_{0}, \Phi_{0}\right)\right) b_{\nu}=-a_{\nu}+(2 \sigma+1)\left(\phi_{\nu}^{2 \sigma} z_{\nu}, \Phi_{0}\right),
$$

and it follows, using (6.6) and (6.3) together with (i) of Lemma 6.1, that

$$
2 \sigma \nu b_{\nu}(1+O(\nu))=a_{\nu}+O\left(\nu^{\frac{2 \sigma+1}{2 \sigma}+1}\right) \text {. }
$$

thus

$$
b_{\nu}=\frac{1}{2 \sigma} C_{*} \nu^{\frac{1}{2 \sigma}-1}+O\left(\nu^{\frac{1}{2 \sigma}}\right),
$$

which completes the proof of (iii).

As for (iv), it suffices to estimate $\left|\partial^{\alpha} y_{\nu}\right|_{L^{2}}$ for all $\alpha$ with $|\alpha|=2$, and then to use (ii).

$$
\left|\partial^{\alpha} y_{\nu}\right|_{L^{2}}^{2}=\left|\partial^{\alpha}\left(\phi_{\nu}-a_{\nu} \Phi_{0}\right)\right|_{L^{2}}^{2} \leq\left|\partial\left(\phi_{\nu}-a_{\nu} \Phi_{0}\right)\right|_{L^{2}}\left|\partial^{\alpha^{\prime}}\left(\phi_{\nu}-a_{\nu} \Phi_{0}\right)\right|_{L^{2}} \leq C \nu^{\frac{2 \sigma+1}{2 \sigma}}
$$

with $\left|\alpha^{\prime}\right|=3$ and $C$ is uniformly bounded in $\nu$; indeed (iv) of Proposition 1 allows us to have $\phi_{\nu} \in C_{\mathrm{loc}}^{3}\left(\mathbb{R}^{2}\right)$ if $\sigma \geq 1$ with its norm uniformly bounded in $\nu$ for $\nu$ sufficiently small. We then use the exponential decay of $\left|\partial^{\alpha^{\prime}} \phi_{\nu}(x)\right|$ for large $|x|$, which may be proved if $\sigma \geq 1$ repeating the same proof of $(\mathrm{v})$ of Proposition 1.

6.2. Spectral Analysis. We investigate the spectral properties of the operator $J \mathcal{L}_{\mu_{0}}$ defined by (1.15). Since a modification of the proof in [17] gives $\operatorname{Ker}\left(L_{\mu_{0}}^{-}\right)=\{0\}$, and it is easily seen that $\operatorname{Ker}\left(L_{\mu_{0}}^{+}\right)=\left\{\phi_{\mu_{0}}\right\}$, one can check, using Eq.(1.17) that

$$
\operatorname{Ker}^{g}\left(J \mathcal{L}_{\mu_{0}}\right)=\operatorname{span}\left\{\left(\begin{array}{c}
\partial_{\mu} \phi_{\mu_{0}} \\
0
\end{array}\right), \quad\left(\begin{array}{c}
0 \\
\phi_{\mu_{0}}
\end{array}\right)\right\} .
$$

For the rest of the spectrum, we write the operator $J \mathcal{L}_{\mu_{0}}$ as follows;

$$
J \mathcal{L}_{\mu_{0}}\left(\begin{array}{cc}
0 & L_{\mu_{0}}^{+} \\
-L_{\mu_{0}}^{-} & 0
\end{array}\right)=J \mathcal{L}_{-\lambda_{0}}+\left(\begin{array}{cc}
0 & \left(\lambda_{0}+\mu_{0}\right)-\phi_{\mu_{0}}^{2 \sigma} \\
-\left(\lambda_{0}+\mu_{0}\right)+(2 \sigma+1) \phi_{\mu_{0}}^{2 \sigma} & 0
\end{array}\right)
$$

where we have defined $J \mathcal{L}_{-\lambda_{0}}$ in the introduction.

Now we consider the case $d=1$. It is well known that the operator $\Xi=-\Delta+|x|^{2}-\lambda_{0}=$ $-\frac{d^{2}}{d x^{2}}+x^{2}-1$ has purely discrete eigenvalues $\tilde{\lambda}_{k}=\lambda_{k}-\lambda_{0}=2 k(k \in \mathbb{N} \cup\{0\})$, and the corresponding eigenfunctions which are the Hermite functions $\Phi_{k}(x)$ (see e.g. [29]) form a complete orthonormal system in $L^{2}(\mathbb{R})$.

For later use, we summarize some properties of the Hermite functions $\Phi_{k}(x)$. For any $k \in$ $\mathbb{N} \cup\{0\}$, the relation

$$
\Phi_{k+1}(x)=\left(-\frac{d}{d x}+x\right) \Phi_{k}(x)
$$

holds between $\Phi_{k+1}$ and $\Phi_{k}$. Thus we have

$$
\begin{aligned}
& \left(\Phi_{n},\left(\frac{d}{d x}+x\right) \Phi_{k}\right)\left(\left(-\frac{d}{d x}+x\right) \Phi_{n}, \Phi_{k}\right)=\left(\Phi_{n+1}, \Phi_{k}\right)=0, \quad \text { if } \quad n+1 \neq k, \\
& \left(\left(-\frac{d}{d x}+x\right) \Phi_{k}, \Phi_{n}\right)=\left(\Phi_{k+1}, \Phi_{n}\right)=0, \quad \text { if } \quad k+1 \neq n .
\end{aligned}
$$


¿From these facts it may be verified that

$$
\left(\Phi_{n}, x \Phi_{k}\right)=0 \quad \text { if } \quad n+1 \neq k \quad \text { and } \quad k+1 \neq n .
$$

It may also be seen that the spectrum of $J \mathcal{L}_{-\lambda_{0}}$ consists only of the discrete eigenvalues

$$
\xi_{k}^{ \pm}\left(-\lambda_{0}\right)= \pm i \tilde{\lambda}_{k}, \quad k \in \mathbb{N} \cup\{0\},
$$

and the (normalized) eigenfunction associated to $\pm i \tilde{\lambda}_{k}$ for $k \neq 0$ may be choosen as

$$
\vec{\Phi}_{k}^{ \pm}=\frac{1}{\sqrt{2}}\left(\begin{array}{c} 
\pm i \Phi_{k} \\
\mp \Phi_{k}
\end{array}\right)
$$

For $k=0$,

$$
\operatorname{Ker}^{g}\left(J \mathcal{L}_{-\lambda_{0}}\right)=\operatorname{Ker}^{g}\left(\mathcal{L}_{-\lambda_{0}} J\right)=\operatorname{span}\left\{\vec{\Phi}_{0}^{-}, \vec{\Phi}_{0}^{+}\right\}, \text {with } \vec{\Phi}_{0}^{-}=\left(\begin{array}{c}
0 \\
\Phi_{0}
\end{array}\right), \vec{\Phi}_{0}^{+}=\left(\begin{array}{c}
\Phi_{0} \\
0
\end{array}\right),
$$

where $\Xi \Phi_{k}=\tilde{\lambda}_{k} \Phi_{k}$ or equivalently $\left(-\Delta+|x|^{2}\right) \Phi_{k}=\lambda_{k} \Phi_{k}(k \in \mathbb{N} \cup\{0\})$ as mentioned above.

Remark 6.1. It may be proved, using a bifurcation argument as follows, that the spectrum of $J \mathcal{L}_{\mu_{0}}$ consists only in pure imaginary discrete, simple eigenvalues (except 0 ) for $\mu_{0}$ sufficiently close to $-\lambda_{0}$. Indeed, fix $k \in \mathbb{N} \cup\{0\}$ and consider for $\mu_{0}$ close to $-\lambda_{0}$, the operators

$$
\Pi_{k}^{ \pm}\left(J \mathcal{L}_{\mu_{0}}\right)=-\frac{1}{2 \pi i} \int_{\Gamma_{k}^{ \pm}}\left(J \mathcal{L}_{\mu_{0}}-z\right)^{-1} d z
$$

with the contour

$$
\Gamma_{k}^{ \pm}=\left\{z \in \mathbb{C},\left|z-\xi_{k}^{ \pm}\left(-\lambda_{0}\right)\right|=1 / 2\right\} .
$$

It follows from Lemma of page 14 in Vol. VI of [25] that if

$$
\left\|\Pi_{k}^{ \pm}\left(J \mathcal{L}_{\mu_{0}}\right)-\Pi_{k}^{ \pm}\left(J \mathcal{L}_{-\lambda_{0}}\right)\right\|_{\mathcal{L}\left(\tilde{\mathbb{L}}^{2}\right)}<1
$$

for $\mu_{0}$ sufficiently close to $-\lambda_{0}$, then

$$
\operatorname{dimRan} \Pi_{k}^{ \pm}\left(J \mathcal{L}_{\mu_{0}}\right)=\operatorname{dimRan} \Pi_{k}^{ \pm}\left(J \mathcal{L}_{-\lambda_{0}}\right) .
$$

In order to prove $(6.12)$, we remark that for any $z \in \rho\left(J \mathcal{L}_{\mu_{0}}\right) \cap \rho\left(J \mathcal{L}_{-\lambda_{0}}\right)$,

$$
\left\|\left(J \mathcal{L}_{\mu_{0}}-z\right)^{-1}-\left(J \mathcal{L}_{-\lambda_{0}}-z\right)^{-1}\right\|_{\mathcal{L}\left(\tilde{\mathbb{L}}^{2}\right)} \leq \sum_{n=1}^{\infty}\left\|J \mathcal{L}_{\mu_{0}}-J \mathcal{L}_{-\lambda_{0}}\right\|_{\mathcal{L}\left(\tilde{\mathbb{L}}^{2}\right)}^{n}\left\|\left(J \mathcal{L}_{-\lambda_{0}}-z\right)^{-1}\right\|_{\mathcal{L}\left(\tilde{\mathbb{L}}^{2}\right)}^{n+1},
$$

and it follows from the spectral theorem that for any $z \in \Gamma_{k}^{ \pm}$,

$$
\left\|\left(J \mathcal{L}_{-\lambda_{0}}-z\right)^{-1}\right\|_{\mathcal{L}\left(\tilde{\mathbb{L}}^{2}\right)}<\frac{1}{\operatorname{dist}\left(z, \sigma\left(J \mathcal{L}_{-\lambda_{0}}\right)\right)}=2 .
$$


Using this, the sum of the series in the right hand side of (6.14) converges for $\mu_{0}$ sufficiently close to $-\lambda_{0}$, since we have for example,

$$
\begin{aligned}
& \left\|J \mathcal{L}_{\mu_{0}}-J \mathcal{L}_{-\lambda_{0}}\right\|_{\mathcal{L}\left(\tilde{\mathbb{L}}^{2}\right)} \\
& =\sup _{|\psi|_{\tilde{\mathbb{L}}^{2}}=1,}\left\{\left|\left(\left(\lambda_{0}+\mu_{0}\right)-\phi_{\mu_{0}}^{2 \sigma}\right) \psi_{I}\right|_{L^{2}}^{2}+\left|\left(\left(\lambda_{0}+\mu_{0}\right)+(2 \sigma+1) \phi_{\mu_{0}}^{2 \sigma}\right) \psi_{R}\right|_{L^{2}}^{2}\right\}^{1 / 2} \\
& \psi=\psi_{R}+i \psi_{I} \in \tilde{\mathbb{L}}^{2} \\
& \leq C(\sigma)\left(\left|\lambda_{0}+\mu_{0}\right|+\left|\phi_{\mu_{0}}\right|_{L^{\infty}}^{2 \sigma}\right) \leq \frac{1}{8},
\end{aligned}
$$

choosing $\mu_{0}$ close enough to $-\lambda_{0}$. We have used Lemma 6.1 and $\Sigma \subset L^{\infty}(\mathbb{R})$ so that $\left|\phi_{\mu_{0}}\right|_{L^{\infty}}^{2 \sigma}$ tends to 0 as $\mu_{0}$ tends to $-\lambda_{0}$. Hence we obtain

$$
\begin{aligned}
\left\|\Pi_{k}^{ \pm}\left(J \mathcal{L}_{\mu_{0}}\right)-\Pi_{k}^{ \pm}\left(J \mathcal{L}_{-\lambda_{0}}\right)\right\|_{\mathcal{L}\left(\tilde{\mathbb{L}}^{2}\right)} & \leq \frac{1}{2 \pi} \int_{\Gamma_{k}^{ \pm}}\left\|\left(J \mathcal{L}_{\mu_{0}}-z\right)^{-1}-\left(J \mathcal{L}_{-\lambda_{0}}-z\right)^{-1}\right\|_{\mathcal{L}\left(\tilde{\mathbb{L}}^{2}\right)}|d z| \\
& \leq \frac{1}{\pi} \sum_{n=1}^{\infty}\left(\frac{1}{4}\right)^{n}\left|\Gamma_{k}^{ \pm}\right|=\frac{1}{3}<1
\end{aligned}
$$

where $\left|\Gamma_{k}^{ \pm}\right|$is the length of $\Gamma_{k}^{ \pm}$.

As a consequence, if $d=1$, all the eigenvalues of $J \mathcal{L}_{\mu_{0}}$, except the zero eigenvalue are discrete, simple and on the imaginary axis since, otherwise, the bifurcation occurs toward two directions due to the symmetry, but it is a contradiction to (6.13).

Remark 6.2. In dimension 2 , denoting $x=\left(x_{1}, x_{2}\right)$, we may write the operator as

$$
-\Delta+|x|^{2}-\lambda_{0}=\left(-\frac{d^{2}}{d x_{1}^{2}}+x_{1}^{2}-1\right) \otimes I+I \otimes\left(-\frac{d^{2}}{d x_{2}^{2}}+x_{2}^{2}-1\right) .
$$

It is known (see for example sections II.4 and VIII.10 of [25]) that the complete orthonormal system in $L^{2}\left(\mathbb{R}^{2}\right)$ associated to the operator $(6.16)$ is

$$
\left\{\Psi_{j, l}(x)\right\}_{j, l \geq 0}=\left\{\Phi_{j}\left(x_{1}\right) \Phi_{l}\left(x_{2}\right)\right\}_{j, l \geq 0},
$$

and the $m$-th eigenvalue of the operator (6.16) is given, for $m \in \mathbb{N} \cup\{0\}$, by

$$
\tilde{\lambda}_{j}+\tilde{\lambda}_{l}=2 m, \text { with } j, l, m \in \mathbb{N} \cup\{0\} \text { and } j+l=m .
$$

The arguments of Remark 6.1 for the operator $J \mathcal{L}_{-\lambda_{0}}$ are still valid in the case $d=2$; we can indeed use Lemma 6.1 (iv) and $\Sigma^{2} \subset L^{\infty}\left(\mathbb{R}^{2}\right)$ for the smallness of $\left|\phi_{\mu_{0}}\right|_{L^{\infty}}^{2 \sigma}$. However, the bifurcation of pure imaginary eigenvalues outside the imaginary axis could occur since the eigenvalues are not simple in 2D. To exclude this possibility, let $\bar{P}$ be the orthogonal projection on $\left(\operatorname{Ker}\left(L_{\mu_{0}}^{+}\right)\right)^{\perp}=\left\{\phi_{\mu_{0}}\right\}^{\perp}$. Since operators $L_{\mu_{0}}^{+}$and $\bar{P} L_{\mu_{0}}^{-} \bar{P}$ have no negative eigenvalue for $\mu_{0}$ close to $-\lambda_{0}$, it follows from Corollary 1.1 in [13] that the bifurcation of eigenvalues cannot happen outside the imaginary axis. 
6.3. Simplified modulation equations. Now the orthogonality conditions (2.5), (2.6), which have been used to estimate the exit time $\tau^{\varepsilon}$ in a convenient way, are not exactly the orthogonality conditions to $\operatorname{Ker}^{g}\left\{J \mathcal{L}_{\mu_{0}}\right\}$. In order to slightly simplify the limit equations, we will now prove that we can change these orthogonality conditions without changing the exit time $\tau^{\varepsilon}$.

Let $P_{\mu_{0}}$ be the spectral projection onto $\operatorname{Ker}^{g}\left(J \mathcal{L}_{\mu_{0}}\right)$ defined by

$$
P_{\mu_{0}} w=\left(\partial_{\mu} \phi_{\mu_{0}}, \phi_{\mu_{0}}\right)^{-1}\left(w_{I}, \partial_{\mu} \phi_{\mu_{0}}\right)\left(\begin{array}{c}
0 \\
\phi_{\mu_{0}}
\end{array}\right)+\left(\partial_{\mu} \phi_{\mu_{0}}, \phi_{\mu_{0}}\right)^{-1}\left(w_{R}, \phi_{\mu_{0}}\right)\left(\begin{array}{c}
\partial_{\mu} \phi_{\mu_{0}} \\
0
\end{array}\right)
$$

for any $w \in L^{2}\left(\mathbb{R}^{d}\right)$ with $\operatorname{Re} w=w_{R}$ and $\operatorname{Im} w=w_{I}$. Set $Q_{\mu_{0}}=I-P_{\mu_{0}}$ and recall that $\eta$ is the solution of (5.1). Then

$$
Q_{\mu_{0}}\left(\begin{array}{c}
0 \\
\phi_{\mu_{0}}
\end{array}\right)=0, \quad d\left(Q_{\mu_{0}} \eta\right)=J \mathcal{L}_{\mu_{0}}\left(Q_{\mu_{0}} \eta\right) d t-Q_{\mu_{0}}\left(\begin{array}{c}
0 \\
|x|^{2} \phi_{\mu_{0}}
\end{array}\right) d W, \quad P_{\mu_{0}} \eta=h(t)\left(\begin{array}{c}
0 \\
\phi_{\mu_{0}}
\end{array}\right),
$$

due to the orthogonality conditions (2.5), (2.6) satisfied by $\eta$. Here, $h(t)$ satisfies the equation

$$
d h=-y d t-L\left(\phi_{\mu_{0}}\right) d W,
$$

with $L\left(\phi_{\mu_{0}}\right)=\left(\partial_{\mu} \phi_{\mu_{0}}, \phi_{\mu_{0}}\right)^{-1}\left(\partial_{\mu} \phi_{\mu_{0}},|x|^{2} \phi_{\mu_{0}}\right)+z(t)$ and $y(t), z(t)$ defined in (5.2).

We put

$$
\begin{aligned}
\tilde{\theta^{\varepsilon}}(t) & =\theta^{\varepsilon}(t)+\varepsilon h(t), \quad \tilde{\mu}^{\varepsilon}(t)=\mu^{\varepsilon}(t), \\
\varepsilon \eta^{\varepsilon}(t, x) & =\varepsilon e^{-i \varepsilon h(t)} \eta^{\varepsilon}(t, x)+e^{-i \varepsilon h(t)} \phi_{\mu^{\varepsilon}(t)}-\phi_{\mu^{\varepsilon}(t)} .
\end{aligned}
$$

Lemma 6.2. Let $T>0$ fixed and $h(t)$ be defined above. Then we have

$$
\lim _{\varepsilon \rightarrow 0} \mathbb{E}\left(\sup _{t \in\left[0, \tau^{\varepsilon} \wedge T\right]} \frac{1}{\varepsilon}\left|e^{-i \varepsilon h(t)} \phi_{\mu^{\varepsilon}(t)}-\phi_{\mu^{\varepsilon}(t)}+i \varepsilon h(t) \phi_{\mu_{0}}\right|_{L^{2}}^{2}\right)=0 .
$$

Proof. Note that $h(t)$ is bounded in $L^{4}\left(\Omega ; L^{\infty}\left(0, T ; L^{2}\left(\mathbb{R}^{d}\right)\right)\right)$ for any $T>0$. Indeed, $\eta_{R}$ is bounded in $L^{4}\left(\Omega ; L^{\infty}\left(0, T ; L^{2}\left(\mathbb{R}^{d}\right)\right)\right)$ by Lemma 5.3 (i) and $\mathbb{E}\left(\sup _{t \leq T} W(t)^{4}\right) \leq C T^{2}$. It follows from Taylor formula that

$$
\begin{aligned}
& \frac{1}{\varepsilon}\left|e^{-i \varepsilon h(t)} \phi_{\mu^{\varepsilon}(t)}-\phi_{\mu^{\varepsilon}(t)}+i \varepsilon h(t) \phi_{\mu_{0}}\right| \\
& =\frac{1}{\varepsilon}\left|\left(e^{-i \varepsilon h(t)}-1+i \varepsilon h(t)\right) \phi_{\mu^{\varepsilon}(t)}+i \varepsilon h(t)\left(\phi_{\mu_{0}}-\phi_{\mu^{\varepsilon}(t)}\right)\right| \\
& \leq \frac{1}{\varepsilon}\left(\left|e^{-i \varepsilon h(t)}-1+i \varepsilon h(t)\right|\left|\phi_{\mu^{\varepsilon}(t)}\right|+\varepsilon|h(t)|\left|\phi_{\mu_{0}}-\phi_{\mu^{\varepsilon}(t)}\right|\right) \\
& \leq \frac{1}{2} \varepsilon h(t)^{2}+|h(t)|\left|\phi_{\mu_{0}}-\phi_{\mu^{\varepsilon}(t)}\right|,
\end{aligned}
$$

the right hand side tends to zero as $\varepsilon$ goes to zero in $L^{2}\left(\Omega ; L^{\infty}\left(0, \tau^{\varepsilon} \wedge T ; L^{2}\left(\mathbb{R}^{d}\right)\right)\right)$ by $(3.3)$, (4.16) and Lemma 5.1 (ii).

Thus letting

$$
\tilde{\eta}(t, x)=\eta(t, x)-i h(t) \phi_{\mu_{0}},
$$

we see that $\tilde{\eta} \varepsilon(t, x)$ converges to $\tilde{\eta}(t, x)$ as $\varepsilon \rightarrow 0$, in probability, in $L^{\infty}\left(0, T ; L^{2}\left(\mathbb{R}^{d}\right)\right)$, where $\tilde{\eta}^{\varepsilon}(t, x)$ is defined by

$$
u^{\varepsilon}(t, x)=e^{i \tilde{\theta}^{\varepsilon}(t)}\left(\phi_{\tilde{\mu}^{\varepsilon}(t)}+\varepsilon \tilde{\eta}^{\varepsilon}(t, x)\right)
$$


Note that $\tilde{\eta}$ satisfies

$$
\begin{aligned}
& P_{\mu_{0}} \tilde{\eta}=0, \quad \tilde{\eta}=\left(I-P_{\mu_{0}}\right) \eta \\
& d \tilde{\eta}=J \mathcal{L}_{\mu_{0}} \tilde{\eta} d t-Q_{\mu_{0}}\left(\begin{array}{c}
0 \\
|x|^{2} \phi_{\mu_{0}}
\end{array}\right) d W
\end{aligned}
$$

which is exactly the equation (2.7). This $\tilde{\eta}^{\varepsilon}(t, x)$ satisfies the conclusion of Theorem 2 with the same exit time $\tau_{\alpha}^{\varepsilon}$ as that for $\eta^{\varepsilon}(t, x)$. The associated modulation parameters $\tilde{\theta}^{\varepsilon}$ and $\tilde{\mu}^{\varepsilon}$ satisfy (2.9), (2.10) and (1.18), as follows from the results of Section 5.

6.4. Proof of Theorem 4. Finally we are in position to prove Theorem 4. We restrict here to the one dimensional case, since the condition $\sigma>1$ is needed. See Remark 6.3 for what can be said in dimension two. Let us consider the projection in $1 \mathrm{D}$, corresponding to $\mu_{0}=-\lambda_{0}, P_{0}$ onto $\operatorname{Ker}\left(J \mathcal{L}_{-\lambda_{0}}\right)$ defined by

$$
P_{0} w\left(w_{I}, \Phi_{0}\right)\left(\begin{array}{c}
0 \\
\Phi_{0}
\end{array}\right)+\left(w_{R}, \Phi_{0}\right)\left(\begin{array}{c}
\Phi_{0} \\
0
\end{array}\right)
$$

for any $w \in L^{2}\left(\mathbb{R}^{d}\right)$ with $\operatorname{Re} w=w_{R}$ and $\operatorname{Im} w=w_{I}$.

Proof of Theorem 4. We obtain for any $\delta \in(0,2)$, using Lemma 6.1 and Eq. (6.18), and setting $\nu=\mu_{0}+\lambda_{0}$ :

$$
\left(I-P_{\mu_{0}}\right)\left(\begin{array}{c}
0 \\
|x|^{2} \phi_{\mu_{0}}
\end{array}\right)=\nu^{\frac{1}{2 \sigma}} C_{*}\left(I-P_{0}\right)\left(\begin{array}{c}
0 \\
|x|^{2} \Phi_{0}
\end{array}\right)+\nu^{\frac{1}{\sigma}} Y_{\nu}
$$

for some $Y_{\nu} \in \tilde{\mathbb{L}^{2}}\left(\mathbb{R}^{d}\right)$ satisfying $\left|Y_{\nu}\right|_{\tilde{\mathbb{L}}^{2}} \leq C$ where $C$ does not depend on $\nu$.

Now we compute the power of each $k$-eigenmode of the process $\tilde{\eta}$ of $(2.7)$. Noting that

$$
\tilde{\eta}(t)=\sum_{k=0}^{\infty} \tilde{\eta}_{k}^{ \pm}(t) \vec{\Phi}_{k}^{ \pm}, \quad \tilde{\eta}_{k}^{ \pm}(t)=\left(\left(\tilde{\eta}(t), \vec{\Phi}_{k}^{ \pm}\right)\right)
$$

we wish to compute the asymptotics as $\nu$ goes to zero of $\mathbb{E}\left(\left|\tilde{\eta}_{k}^{ \pm}(t)\right|^{2}\right)$. For this aim, we change the time scale by setting

$$
s=\nu^{1 / \sigma} t
$$

Then Eq.(2.7) is written as

$$
d \tilde{\eta}=\nu^{-\frac{1}{\sigma}} J \mathcal{L}_{\mu_{0}} \tilde{\eta} d s-\nu^{-\frac{1}{2 \sigma}}\left(I-P_{\mu_{0}}\right)\left(\begin{array}{c}
0 \\
|x|^{2} \phi_{\mu_{0}}
\end{array}\right) d \tilde{W}(s)
$$

with the new standard Brownian motion $\tilde{W}(s)=\nu^{1 / 2 \sigma} W\left(\nu^{-1 / \sigma} s\right)$. By the definition of $P_{0}$,

$$
\left(I-P_{0}\right)\left(\begin{array}{c}
0 \\
|x|^{2} \Phi_{0}
\end{array}\right)\left(\begin{array}{c}
0 \\
|x|^{2} \Phi_{0}-\left(|x|^{2} \Phi_{0}, \Phi_{0}\right) \Phi_{0}
\end{array}\right) .
$$

Together with (6.20), we have

$$
d \tilde{\eta}=\nu^{-\frac{1}{\sigma}} J \mathcal{L}_{-\lambda_{0}} \tilde{\eta} d s+\nu^{-\frac{1}{\sigma}} B_{\nu} \tilde{\eta} d s-C_{*}\left(\begin{array}{c}
0 \\
|x|^{2} \Phi_{0}-\left|x \Phi_{0}\right|_{L^{2}}^{2} \Phi_{0} \\
32
\end{array}\right) d \tilde{W}(s)-\nu^{\frac{1}{2 \sigma}} Y_{\nu} d \tilde{W}(s),
$$


where

$$
B_{\nu}=\left(\begin{array}{cc}
0 & \nu-\phi_{\mu_{0}}^{2 \sigma} \\
-\nu+(2 \sigma+1) \phi_{\mu_{0}}^{2 \sigma} & 0
\end{array}\right)
$$

We take the $\tilde{\mathbb{L}}^{2}$ product of Eq. (6.23) with $\vec{\Phi}_{k}^{ \pm}$. If $k=0$, since $\tilde{\lambda}_{0}=0$,

$$
d \tilde{\eta}_{0}^{ \pm}=\nu^{-\frac{1}{\sigma}}\left(\left(B_{\nu} \tilde{\eta}, \vec{\Phi}_{0}^{ \pm}\right)\right) d s-\nu^{\frac{1}{2 \sigma}}\left(\left(Y_{\nu}, \vec{\Phi}_{0}^{ \pm}\right)\right) d \tilde{W}(s) .
$$

Itô formula for $\left|\tilde{\eta}_{0}^{ \pm}\right|^{2}$ leads to

$$
d\left|\tilde{\eta}_{0}^{ \pm}\right|^{2}=2 \nu^{-\frac{1}{\sigma}} \operatorname{Re}\left(\overline{\tilde{\eta}}_{0}^{ \pm}\left(\left(B_{\nu} \tilde{\eta}, \vec{\Phi}_{0}^{ \pm}\right)\right)\right) d s-2 \nu^{\frac{1}{2 \sigma}} \operatorname{Re}\left(\overline{\tilde{\eta}}_{0}^{ \pm}\left(\left(Y_{\nu}, \vec{\Phi}_{0}^{ \pm}\right)\right)\right) d \tilde{W}(s)+\nu^{\frac{1}{\sigma}}\left|\left(\left(Y_{\nu}, \vec{\Phi}_{0}^{ \pm}\right)\right)\right|^{2} d s .
$$

In the same way, noting that $J \mathcal{L}_{-\lambda_{0}}=\mathcal{L}_{-\lambda_{0}} J$, we get for $k \geq 1$,

$$
\begin{aligned}
d \tilde{\eta}_{k}^{ \pm}= & \mp i \tilde{\lambda}_{k} \nu^{-1 / \sigma} \tilde{\eta}_{k}^{ \pm}(s) d s+\nu^{-1 / \sigma}\left(\left(B_{\nu} \tilde{\eta}, \vec{\Phi}_{k}^{ \pm}\right)\right) d s \\
& -\frac{C_{*}}{\sqrt{2}}\left(|x|^{2} \Phi_{0}, \mp \Phi_{k}\right) d \tilde{W}(s)-\nu^{1 / 2 \sigma}\left(\left(Y_{\nu}, \vec{\Phi}_{k}^{ \pm}\right)\right) d \tilde{W}(s) .
\end{aligned}
$$

Then, again with the Itô formula,

$$
\begin{aligned}
d\left|\tilde{\eta}_{k}^{ \pm}\right|^{2}= & \frac{1}{2} C_{*}^{2}\left(|x|^{2} \Phi_{0}, \Phi_{k}\right)^{2} d s \\
& +\sqrt{2} \nu^{-\frac{1}{\sigma}}\left\{\left(\operatorname{Im} \tilde{\eta}, \mp \Phi_{k}\right) \operatorname{Re}\left(\left(B_{\nu} \tilde{\eta}, \vec{\Phi}_{k}^{ \pm}\right)\right)-\left(\operatorname{Re} \tilde{\eta}, \pm \Phi_{k}\right) \operatorname{Im}\left(\left(B_{\nu} \tilde{\eta}, \vec{\Phi}_{k}^{ \pm}\right)\right)\right\} d s \\
& +\sqrt{2} C_{*} \nu^{\frac{1}{2 \sigma}}\left(|x|^{2} \Phi_{0}, \mp \Phi_{k}\right) \operatorname{Re}\left(\left(Y_{\nu}, \vec{\Phi}_{k}^{ \pm}\right)\right) d s+\nu^{\frac{1}{\sigma}}\left|\operatorname{Re}\left(\left(Y_{\nu}, \vec{\Phi}_{k}^{ \pm}\right)\right)\right|^{2} d s \\
& -C_{*}\left(\operatorname{Im} \tilde{\eta}, \mp \Phi_{k}\right)\left(|x|^{2} \Phi_{0}, \mp \Phi_{k}\right) d \tilde{W}(s)+\sqrt{2} \nu^{\frac{1}{2 \sigma}}\left\{\left(\operatorname{Re} \tilde{\eta}, \pm \Phi_{k}\right) \operatorname{Im}\left(\left(Y_{\nu}, \vec{\Phi}_{k}^{ \pm}\right)\right)\right. \\
& \left.-\left(\operatorname{Im} \tilde{\eta}, \mp \Phi_{k}\right) \operatorname{Re}\left(\left(Y_{\nu}, \vec{\Phi}_{k}^{ \pm}\right)\right)\right\} d \tilde{W}(s) .
\end{aligned}
$$

We recall that $\tilde{\eta}(0)=0$. Note also that by $(6.15)$ and Lemma 6.1 (i),

$$
\left\|B_{\nu}\right\|_{\mathcal{L}\left(\mathbb{L}^{2}\right)} \leq C \nu
$$

for some positive constant $C$. Hence, using Lemma 5.3 (i),

$$
\begin{aligned}
& \mathbb{E}\left(\sqrt{2} \nu^{-\frac{1}{\sigma}} \int_{0}^{t}\left|\left(\operatorname{Im} \tilde{\eta}, \mp \Phi_{k}\right) \operatorname{Re}\left(\left(B_{\nu} \tilde{\eta}, \vec{\Phi}_{k}^{ \pm}\right)\right)-\left(\operatorname{Re} \tilde{\eta}, \pm \Phi_{k}\right) \operatorname{Im}\left(\left(B_{\nu} \tilde{\eta}, \vec{\Phi}_{k}^{ \pm}\right)\right)\right|\right) d s \\
& \leq \sqrt{2} \nu^{-\frac{1}{\sigma}} \mathbb{E}\left(\int_{0}^{t}\left\|B_{\nu}\right\|_{\mathcal{L}\left(\mathbb{L}^{2}\right)}|\tilde{\eta}(s)|_{L^{2}}^{2} d s\right) \leq 2 C \nu^{1-\frac{1}{\sigma}} \int_{0}^{t} \mathbb{E}\left(\sup _{s \leq T}|\tilde{\eta}(s)|_{L^{2}}^{2}\right) d s \leq C \nu^{1-\frac{1}{\sigma}} t .
\end{aligned}
$$

Integrating Eqs (6.24) and (6.25) on $[0, t \wedge T]$ and taking the expectation, we obtain

$$
\begin{aligned}
& \mathbb{E}\left(\left|\tilde{\eta}_{k}^{ \pm}(t)\right|^{2}\right)=\frac{1}{2} C_{*}^{2}\left(|x|^{2} \Phi_{0}, \mp \Phi_{k}\right)^{2} t+O\left(\nu^{\kappa} t\right), \text { if } k \geq 1, \\
& \mathbb{E}\left(\left|\tilde{\eta}_{0}^{ \pm}(t)\right|^{2}\right)=O\left(\nu^{\kappa} t\right),
\end{aligned}
$$

where $\kappa=\min \left\{\frac{1}{2 \sigma}, 1-\frac{1}{\sigma}\right\}$. On the other hand, noting that for any $k \geq 1$

$$
\left(|x|^{2} \Phi_{0}, \Phi_{k}\right)=\frac{1}{2}\left(x \Phi_{0}, \Phi_{k+1}\right)+\frac{1}{2}\left(\Phi_{1}, x \Phi_{k}\right),
$$

it follows from (6.9) that

$$
\left(|x|^{2} \Phi_{0}, \Phi_{k}\right)=0, \text { except for } k=2, \quad \text { and } \quad\left(|x|^{2} \Phi_{0}, \Phi_{2}\right)=\frac{1}{2}\left(x \Phi_{1}, \Phi_{2}\right) .
$$


Indeed, by (6.8), if $k \neq 0$, we get

$$
\begin{aligned}
\left(x^{2} \Phi_{0}, \Phi_{k}\right) & =-\left(\partial_{x} \Phi_{0}, \partial_{x} \Phi_{k}\right)=-\left(x \Phi_{0}-\Phi_{1}, x \Phi_{k}-\Phi_{k+1}\right) \\
& =-\left(x^{2} \Phi_{0}, \Phi_{k}\right)+\left(x \Phi_{0}, \Phi_{k+1}\right)+\left(\Phi_{1}, x \Phi_{k}\right) .
\end{aligned}
$$

These computations finally lead to

$$
\begin{aligned}
& \mathbb{E}\left(\left|\tilde{\eta}_{2}^{ \pm}(t)\right|^{2}\right)=\frac{C_{*}^{2}}{2}\left(x \Phi_{1}, \Phi_{2}\right)^{2} t+O\left(\nu_{0}^{\kappa} t\right), \\
& \mathbb{E}\left(\left|\tilde{\eta}_{k}^{ \pm}(t)\right|^{2}\right)=O\left(\nu_{0}^{\kappa} t\right) \text { if } k \neq 2 .
\end{aligned}
$$

This is the master equation in the case $d=1$. Note that this does not mean anything if $\sigma=1$. Recall $\nu=\lambda_{0}+\mu_{0}$. To complete the statement in Theorem 4, it suffices to remark that $\left(x \Phi_{1}, \Phi_{2}\right)=-1$.

Remark 6.3. In the case $d=2$, the above arguments would work up to Eq.(6.26), setting (see Eq. (6.17))

$$
\tilde{\eta}(t)=\sum_{j, l=0}^{\infty} \tilde{\eta}_{j, l}^{ \pm}(t) \vec{\Psi}_{j, l}^{ \pm}, \quad \tilde{\eta}_{j, l}^{ \pm}(t)=\left(\left(\tilde{\eta}(t), \vec{\Psi}_{j, l}^{ \pm}\right)\right),
$$

provided that we could take $\sigma>1$. We could also compute the quantity $\left(|x|^{2} \Psi_{0,0}, \Psi_{j, l}\right)$ for $j+l=m$ and $m \geq 1$. Indeed, by (6.17), and with $x=\left(x_{1}, x_{2}\right)$, we have

$$
\left(|x|^{2} \Psi_{0,0}, \Psi_{j, l}\right)=\left(\left(x_{1}^{2}+x_{2}^{2}\right) \Phi_{0}\left(x_{1}\right) \Phi_{0}\left(x_{2}\right), \Phi_{l}\left(x_{1}\right) \Phi_{j}\left(x_{2}\right)\right)=\delta_{0 j}\left(x_{1}^{2} \Phi_{0}, \Phi_{l}\right)_{x_{1}}+\delta_{0 l}\left(x_{2}^{2} \Phi_{0}, \Phi_{j}\right)_{x_{2}}
$$

for $j+l=m$ and $m \geq 1$. Then, using (6.27),

$$
\left(|x|^{2} \Psi_{0,0}, \Psi_{j, l}\right)=\left\{\begin{array}{l}
\frac{1}{2}\left(x_{1} \Phi_{1}, \Phi_{2}\right) \text { if }(j, l) \in\{(0,2),(2,0)\} \\
0, \text { otherwise }
\end{array}\right.
$$

As a consequence, we would obtain the following master equation (in the new time scale (6.21)) in $2 \mathrm{D}$ :

$$
\begin{aligned}
& \mathbb{E}\left(\left|\tilde{\eta}_{j, l}^{ \pm}(s)\right|^{2}\right)=\frac{C_{*}^{2}}{8}\left(x_{1} \Phi_{1}, \Phi_{2}\right)^{2} s+O\left(\nu^{\kappa} s\right), \text { if }(j, l) \in\{(0,2),(2,0)\} \\
& \mathbb{E}\left(\left|\tilde{\eta}_{j, l}^{ \pm}(s)\right|^{2}\right)=O\left(\nu^{\kappa} s\right) \text { if }(j, l) \notin\{(0,2),(2,0)\} .
\end{aligned}
$$

Acknowledgements. The first author was partially supported by the ANR research project "Dispersive PDEs" (ANR-07-BLAN-0250) and the second author was partially supported by JSPS Postdoctoral Fellowships for Research Abroad.

\section{APPENDIX}

In this section we will prove the estimates in Lemmas 5.1 and 5.3. All the computations in this section applying Itô formula may be justified with a similar method to the proof of Lemma 4.1. We begin with (i) of Lemma 5.1. 
Proof of Lemma 5.1 ( $i$ ). Itô formula leads the equations

$$
\begin{aligned}
d\left|\eta_{R}^{\varepsilon}\right|_{L^{2}}^{2}+d\left|\eta_{I}^{\varepsilon}\right|_{L^{2}}^{2}= & 4 \sigma\left(\eta_{I}^{\varepsilon}, \phi_{\mu_{0}}^{2 \sigma} \eta_{R}^{\varepsilon}\right) d t+\left.\left.|| x\right|^{2} \phi_{\mu^{\varepsilon}}\right|_{L^{2}} ^{2} d t+\left(z^{\varepsilon}\right)^{2}\left|\phi_{\mu^{\varepsilon}}\right|_{L^{2}}^{2} d t+2 z^{\varepsilon}\left|x \phi_{\mu^{\varepsilon}}\right|_{L^{2}}^{2} d t \\
& -2 a^{\varepsilon}\left(\eta_{R}^{\varepsilon}, \partial_{\mu} \phi_{\mu^{\varepsilon}}\right) d t+\left(b^{\varepsilon}\right)^{2}\left|\partial_{\mu} \phi_{\mu^{\varepsilon}}\right|_{L^{2}}^{2} d t-2 y^{\varepsilon}\left(\eta_{I}^{\varepsilon}, \phi_{\mu^{\varepsilon}}\right) d t \\
& +2(2 \sigma+1)\left(\eta_{I}^{\varepsilon},\left(\phi_{\mu^{\varepsilon}}^{2 \sigma}-\phi_{\mu_{0}}^{2 \sigma}\right) \eta_{R}^{\varepsilon}\right) d t+2\left(\eta_{R}^{\varepsilon},\left(\phi_{\mu_{0}}^{2 \sigma}-\phi_{\mu^{\varepsilon}}^{2 \sigma}\right) \eta_{I}^{\varepsilon}\right) d t \\
& -2 \varepsilon z^{\varepsilon} b^{\varepsilon}\left(\eta_{I}^{\varepsilon}, \partial_{\mu} \phi_{\mu^{\varepsilon}}\right) d t-\varepsilon\left(\eta_{R}^{\varepsilon},|x|^{4} \phi_{\mu^{\varepsilon}}\right) d t-\varepsilon\left(z^{\varepsilon}\right)^{2}\left(\eta_{R}^{\varepsilon}, \phi_{\mu^{\varepsilon}}\right) d t \\
& -\varepsilon\left(b^{\varepsilon}\right)^{2}\left(\eta_{R}^{\varepsilon}, \partial_{\mu}^{2} \phi_{\mu^{\varepsilon}}\right) d t-2 \varepsilon b^{\varepsilon}\left(|x|^{2} \eta_{I}^{\varepsilon}, \partial_{\mu} \phi_{\mu^{\varepsilon}}\right) d t+2 \varepsilon\left(|x|^{4} \phi_{\mu^{\varepsilon}}, \eta_{R}^{\varepsilon}\right) d t \\
& +2 \varepsilon z^{\varepsilon}\left(|x|^{2} \phi_{\mu^{\varepsilon}}, \eta_{R}^{\varepsilon}\right) d t+2 \varepsilon\left(z^{\varepsilon}\right)^{2}\left(\phi_{\mu^{\varepsilon}}, \eta_{R}^{\varepsilon}\right) d t-2 \varepsilon\left(\eta_{R}^{\varepsilon}, h_{I}^{\varepsilon}\right) d t+2 \varepsilon\left(\eta_{I}^{\varepsilon}, h_{R}^{\varepsilon}\right) d t \\
& -2 b^{\varepsilon}\left(\eta_{R}^{\varepsilon}, \partial_{\mu} \phi_{\mu^{\varepsilon}}\right) d W-2\left(\eta_{I}^{\varepsilon},|x|^{2} \phi_{\mu^{\varepsilon}}\right) d W-2 z^{\varepsilon}\left(\eta_{I}^{\varepsilon}, \phi_{\mu^{\varepsilon}}\right) d W .
\end{aligned}
$$

We note that

$$
\begin{aligned}
\left|-2 \varepsilon\left(\eta_{R}^{\varepsilon}, h_{I}^{\varepsilon}\right)+2 \varepsilon\left(\eta_{I}^{\varepsilon}, h_{R}^{\varepsilon}\right)\right| & \leq\left(\varepsilon\left|\eta^{\varepsilon}\right|^{2}\left(\left|\phi_{\mu^{\varepsilon}}\right|^{2 \sigma-1}+\left|\varepsilon \eta^{\varepsilon}\right|^{2 \sigma-1}\right),\left|\eta^{\varepsilon}\right|\right) \\
& \leq\left|\eta^{\varepsilon}\right|_{L^{2}}^{2}\left|\varepsilon \eta^{\varepsilon}\right|_{L^{\infty}}\left(\left|\phi_{\mu^{\varepsilon}}\right|_{L^{\infty}}^{2 \sigma-1}+\left|\varepsilon \eta^{\varepsilon}\right|_{L^{\infty}}^{2 \sigma-1}\right) \\
& \leq C\left|\eta^{\varepsilon}\right|_{L^{2}}^{2}
\end{aligned}
$$

where $C$ depends only on $\alpha, \sigma, \mu_{0}$ and moreover on $N$ if $d=2$ for $t \leq \tilde{\tau}^{\varepsilon} \wedge T$. It follows from Eqs. (4.13) and (4.14) that a.s. for $t \leq \tilde{\tau}^{\varepsilon} \wedge T$, putting $\tau=t \wedge \tilde{\tau}^{\varepsilon}$,

$$
\left|\eta_{R}^{\varepsilon}(t)\right|_{L^{2}}^{2}+\left|\eta_{I}^{\varepsilon}(t)\right|_{L^{2}}^{2} \leq C \int_{0}^{\tau}\left(1+\left|\eta_{R}^{\varepsilon}(s)\right|_{L^{2}}^{2}+\left|\eta_{I}^{\varepsilon}(s)\right|_{L^{2}}^{2}\right) d s+\left|\int_{0}^{\tau} M(s) d W(s)\right|
$$

with

$$
|M(s)| \leq C^{\prime}\left(1+\left|\eta_{R}^{\varepsilon}(s)\right|_{L^{2}}+\left|\eta_{I}^{\varepsilon}(s)\right|_{L^{2}}\right), \text { a.s. for } s \leq \tau .
$$

Indeed we may use Proposition 1 (i), (iv), and (v). Taking the expectation of the supremum in time and using a martingale inequality, we get

$$
\begin{aligned}
\mathbb{E}\left(\sup _{t \leq \tilde{\tau}^{\varepsilon} \wedge T}\left|\eta^{\varepsilon}(t)\right|_{L^{2}}^{2}\right) & \leq C \mathbb{E}\left(\int_{0}^{\tilde{\tau}^{\varepsilon} \wedge T} \sup _{s \leq \tilde{\tau}^{\varepsilon} \wedge T}\left(1+\left|\eta^{\varepsilon}(s)\right|_{L^{2}}^{2}\right)\right)+C^{\prime} \mathbb{E}\left(\int_{0}^{\tilde{\tau}^{\varepsilon} \wedge T}\left(1+\left|\eta^{\varepsilon}(t)\right|_{L^{2}}^{2}\right) d t\right)^{1 / 2} \\
& \leq C \mathbb{E}\left(\int_{0}^{T} \sup _{s \leq \tilde{\tau}^{\varepsilon} \wedge T}\left(1+\left|\eta^{\varepsilon}(s)\right|_{L^{2}}^{2}\right)\right)+C^{\prime} T^{1 / 2} \mathbb{E}\left(\sup _{t \leq \tilde{\tau}^{\varepsilon} \wedge T}\left(1+\left|\eta^{\varepsilon}(t)\right|_{L^{2}}^{2}\right)\right)^{1 / 2}
\end{aligned}
$$

from which, by Gronwall inequality,

$$
\mathbb{E}\left(\sup _{t \leq \tilde{\tau}^{\varepsilon} \wedge T}\left|\eta^{\varepsilon}(t)\right|_{L^{2}}^{2}\right) \leq C_{1}(T)
$$

follows.

Proof of Lemma 5.1 (ii). We apply Ito formula and we have

$$
d\left|\eta^{\varepsilon}(t)\right|_{L^{2}}^{4}=2\left(\left|\eta^{\varepsilon}(t)\right|_{L^{2}}^{2}, d\left|\eta^{\varepsilon}(t)\right|_{L^{2}}^{2}\right)+\left|2 b^{\varepsilon}\left(\eta_{R}^{\varepsilon}, \partial_{\mu} \phi_{\mu^{\varepsilon}}\right)+2\left(\eta_{I}^{\varepsilon},|x|^{2} \phi_{\mu^{\varepsilon}}\right)+2 z^{\varepsilon}\left(\eta_{I}^{\varepsilon}, \phi_{\mu^{\varepsilon}}\right)\right|^{2} d t .
$$

We use the previous computations :

$$
\left|\eta^{\varepsilon}(t)\right|_{L^{2}}^{4} \leq C \int_{0}^{\tau}\left(1+\left|\eta^{\varepsilon}(t)\right|_{L^{2}}^{4}\right) d t+\left|\int_{0}^{\tau} M^{\prime}(s) d W(s)\right|
$$

a.s. for $t \leq \tau$, with

$$
\left|M^{\prime}(s)\right| \leq C^{\prime}\left(1+\left|\eta^{\varepsilon}(s)\right|_{L^{2}}^{3}\right), \text { a.s. for } s \leq \tau \text {. }
$$


Then, integrating Eq. (7.1), taking the expectation and using Gronwall Lemma, we have

$$
\sup _{t \leq T} \mathbb{E}\left(\mathbb{1}_{\left[0, \tilde{\tau}^{\varepsilon}\right)}(t)\left|\eta^{\varepsilon}(t)\right|_{L^{2}}^{4}\right) \leq C(T)
$$

Hence,

$$
\mathbb{E}\left(\sup _{t \leq \tilde{\tau}^{\varepsilon} \wedge T}\left|\eta^{\varepsilon}(t)\right|_{L^{2}}^{4}\right) \leq C+C^{\prime} \mathbb{E}\left(\sup _{t \leq \tilde{\tau}^{\varepsilon} \wedge T}\left|\int_{0}^{\tau} M^{\prime}(s) d W(s)\right|\right),
$$

and by (7.2) and the martingale inequality given by Theorem 3.14 in [5], we have

$$
\begin{aligned}
\mathbb{E}\left(\sup _{t \leq \tilde{\tau}^{\varepsilon} \wedge T}\left|\int_{0}^{t \wedge \tau} M^{\prime}(s) d W(s)\right|\right) & \leq 3 \mathbb{E}\left(\left(\int_{0}^{T \wedge \tilde{\tau}^{\varepsilon}}\left(1+\left|\eta^{\varepsilon}(s)\right|^{6}\right) d s\right)^{1 / 2}\right) \\
& \leq 3 T^{1 / 2} \mathbb{E}\left(\sup _{t \leq \tilde{\tau}^{\varepsilon} \wedge T}\left(1+\left|\eta^{\varepsilon}(t)\right|_{L^{2}}^{3}\right)\right) \\
& \leq \frac{1}{2} \mathbb{E}\left(\sup _{t \leq \tilde{\tau}^{\varepsilon} \wedge T}\left|\eta^{\varepsilon}(t)\right|_{L^{2}}^{4}\right)+C(T),
\end{aligned}
$$

where we have used Young inequality in the last inequality. Finally we obtain

$$
\mathbb{E}\left(\sup _{t \leq \tilde{\tau}^{\varepsilon} \wedge T}\left|\eta^{\varepsilon}(t)\right|_{L^{2}}^{4}\right) \leq C(\alpha, T)
$$

Proof of Lemma 5.2. Itô formula to $\left(L_{\mu_{0}}^{-} \eta_{R}^{\varepsilon}, \eta_{R}^{\varepsilon}\right)+\left(L_{\mu_{0}}^{+} \eta_{I}^{\varepsilon}, \eta_{I}^{\varepsilon}\right)$ results in, taking account of some compensations,

$$
\begin{aligned}
& d\left(\left(L_{\mu_{0}}^{-} \eta_{R}^{\varepsilon}, \eta_{R}^{\varepsilon}\right)+\left(L_{\mu_{0}}^{+} \eta_{I}^{\varepsilon}, \eta_{I}^{\varepsilon}\right)\right) \\
& -4 \sigma\left(\mu^{\varepsilon}-\mu_{0}\right)\left(\phi_{\mu_{0}}^{2 \sigma} \eta_{R}^{\varepsilon}, \eta_{I}^{\varepsilon}\right) d t+2\left(L_{\mu_{0}}^{-} \eta_{R}^{\varepsilon},\left(\phi_{\mu_{0}}^{2 \sigma}-\phi_{\mu^{\varepsilon}}^{2 \sigma}\right) \eta_{I}^{\varepsilon}\right) d t \\
& -2(2 \sigma+1)\left(L_{\mu_{0}}^{+} \eta_{I}^{\varepsilon},\left(\phi_{\mu^{\varepsilon}}^{2 \sigma}-\phi_{\mu_{0}}^{2 \sigma}\right) \eta_{R}^{\varepsilon}\right) d t-2 a^{\varepsilon}\left(L_{\mu_{0}}^{-} \eta_{R}^{\varepsilon}, \partial_{\mu} \phi_{\mu^{\varepsilon}}\right) d t-2 y^{\varepsilon}\left(\eta_{I}^{\varepsilon}, L_{\mu_{0}}^{+} \phi_{\mu^{\varepsilon}}\right) d t \\
& +\left(b^{\varepsilon}\right)^{2}\left(L_{\mu_{0}}^{-}\left(\partial_{\mu} \phi_{\mu^{\varepsilon}}\right), \partial_{\mu} \phi_{\mu^{\varepsilon}}\right) d t+\left(L_{\mu_{0}}^{+}\left(|x|^{2} \phi_{\mu^{\varepsilon}}\right),|x|^{2} \phi_{\mu^{\varepsilon}}\right) d t+2 z^{\varepsilon}\left(L_{\mu_{0}}^{+}\left(|x|^{2} \phi_{\mu^{\varepsilon}}\right), \phi_{\mu^{\varepsilon}}\right) d t \\
& +\left(z^{\varepsilon}\right)^{2}\left(L_{\mu_{0}}^{+}\left(\phi_{\mu^{\varepsilon}}\right), \phi_{\mu^{\varepsilon}}\right) d t-4 \sigma \varepsilon y^{\varepsilon}\left(\eta_{R}^{\varepsilon}, \eta_{I}^{\varepsilon}\right) d t-\varepsilon\left(L_{\mu_{0}}^{-} \eta_{R}^{\varepsilon},|x|^{4} \phi_{\mu^{\varepsilon}}\right) d t+4 \sigma \varepsilon z^{\varepsilon}\left(\phi_{\mu_{0}}^{2 \sigma} \eta_{R}^{\varepsilon},|x|^{2} \phi_{\mu^{\varepsilon}}\right) d t \\
& +2 \sigma \varepsilon\left(z^{\varepsilon}\right)^{2}\left(\phi_{\mu_{0}}^{2 \sigma} \eta_{R}^{\varepsilon}, \phi_{\mu^{\varepsilon}}\right) d t+\varepsilon\left(z^{\varepsilon}\right)^{2}\left(\eta_{R}^{\varepsilon}, L_{\mu_{0}}^{+}\left(\phi_{\mu^{\varepsilon}}\right)\right) d t-\varepsilon\left(b^{\varepsilon}\right)^{2}\left(\eta_{R}^{\varepsilon}, L_{\mu_{0}}^{-}\left(\partial_{\mu}^{2} \phi_{\mu^{\varepsilon}}\right)\right) d t \\
& -2 \varepsilon\left(L_{\mu_{0}}^{-} \eta_{R}^{\varepsilon}, h_{I}^{\varepsilon}\right) d t+2 \varepsilon\left(L_{\mu_{0}}^{+} \eta_{I}^{\varepsilon}, h_{R}^{\varepsilon}\right) d t-2 \varepsilon b^{\varepsilon}\left(L_{\mu_{0}}^{-}\left(\partial_{\mu} \phi_{\mu^{\varepsilon}}\right),|x|^{2} \eta_{I}^{\varepsilon}\right) d t-2 \varepsilon b^{\varepsilon} z^{\varepsilon}\left(L_{\mu_{0}}^{-}\left(\partial_{\mu} \phi_{\mu^{\varepsilon}}\right), \eta_{I}^{\varepsilon}\right) d t \\
& +2 \varepsilon\left(|x|^{2} L_{\mu_{0}}^{+}\left(|x|^{2} \phi_{\mu^{\varepsilon}}\right), \eta_{R}^{\varepsilon}\right) d t+2 \varepsilon z^{\varepsilon}\left(|x|^{2} L_{\mu_{0}}^{+}\left(\phi_{\mu^{\varepsilon}}\right), \eta_{R}^{\varepsilon}\right) d t+2 \sigma \varepsilon^{2}\left(|x|^{4} \phi_{\mu_{0}}^{2 \sigma} \eta_{R}^{\varepsilon}, \eta_{R}^{\varepsilon}\right) d t \\
& -(6-2 d) \varepsilon^{2}\left|x \eta_{R}^{\varepsilon}\right|_{L^{2}}^{2} d t+4 \sigma \varepsilon^{2} z^{\varepsilon}\left(\phi_{\mu_{0}}^{2 \sigma} \eta_{R}^{\varepsilon},|x|^{2} \eta_{R}^{\varepsilon}\right) d t+2 \sigma \varepsilon^{2}\left(z^{\varepsilon}\right)^{2}\left(\phi_{\mu_{0}}^{2 \sigma} \eta_{R}^{\varepsilon}, \eta_{R}^{\varepsilon}\right) d t \\
& -2 \sigma \varepsilon^{2}\left(|x|^{4} \phi_{\mu_{0}}^{2 \sigma} \eta_{I}^{\varepsilon}, \eta_{I}^{\varepsilon}\right) d t+(6-2 d) \varepsilon^{2}\left|x \eta_{I}^{\varepsilon}\right|_{L^{2}}^{2} d t-4 \sigma \varepsilon^{2} z^{\varepsilon}\left(|x|^{2} \phi_{\mu_{0}}^{2 \sigma} \eta_{I}^{\varepsilon}, \eta_{I}^{\varepsilon}\right) d t \\
& -2 \sigma \varepsilon^{2}\left(z^{\varepsilon}\right)^{2}\left(\phi_{\mu_{0}}^{2 \sigma} \eta_{I}^{\varepsilon}, \eta_{I}^{\varepsilon}\right) d t-2 b^{\varepsilon}\left(\eta_{R}^{\varepsilon}, L_{\mu_{0}}^{\varepsilon}, L_{\mu_{0}}^{+} \phi_{\mu^{\varepsilon}}\right) d W-8 \varepsilon\left(\eta_{R}^{\varepsilon}, x \cdot \nabla \eta_{I}^{\varepsilon}\right) d W-4 d \varepsilon\left(\eta_{R}^{\varepsilon}, \eta_{I}^{\varepsilon}\right) d W \\
& +4 \sigma \varepsilon\left(\phi_{\mu_{0}}^{2 \sigma} \eta_{R}^{\varepsilon}, \eta_{I}^{\varepsilon}\right) d W-4 \sigma \varepsilon z^{\varepsilon}\left(\phi_{\mu_{0}}^{2 \sigma} \eta_{R}^{\varepsilon}, \eta_{I}^{\varepsilon}\right) d W \\
& 36
\end{aligned}
$$


We set

$$
\begin{aligned}
D_{3}(t)= & -4 \sigma\left(\mu^{\varepsilon}-\mu_{0}\right)\left(\phi_{\mu_{0}}^{2 \sigma} \eta_{R}^{\varepsilon}, \eta_{I}^{\varepsilon}\right)-4 \sigma \varepsilon y^{\varepsilon}\left(\eta_{R}^{\varepsilon}, \eta_{I}^{\varepsilon}\right) \\
& -2 a^{\varepsilon}\left(L_{\mu_{0}}^{-} \eta_{R}^{\varepsilon}, \partial_{\mu} \phi_{\mu^{\varepsilon}}\right)-2 y^{\varepsilon}\left(\eta_{I}^{\varepsilon}, L_{\mu_{0}}^{+} \phi_{\mu^{\varepsilon}}\right) \\
D_{4}(t)= & -2 \varepsilon\left(L_{\mu_{0}}^{-} \eta_{R}^{\varepsilon}, h_{I}^{\varepsilon}\right)+2 \varepsilon\left(L_{\mu_{0}}^{+} \eta_{I}^{\varepsilon}, h_{R}^{\varepsilon}\right) \\
D_{5}(t)= & \text { the rest of drift terms } \\
M_{2}(t)= & \text { martingale parts }
\end{aligned}
$$

and we have a.s., for $t \leq \tilde{\tau}^{\varepsilon} \wedge T$, putting $\tau=t \wedge \tilde{\tau}^{\varepsilon}$,

$$
\left(L_{\mu_{0}}^{-} \eta_{R}^{\varepsilon}(t), \eta_{R}^{\varepsilon}(t)\right)+\left(L_{\mu_{0}}^{+} \eta_{I}^{\varepsilon}(t), \eta_{I}^{\varepsilon}(t)\right)=\int_{0}^{\tau}\left(D_{3}(s)+D_{4}(s)+D_{5}(s)\right) d s+\int_{0}^{\tau} M_{2}(s) d W(s) .
$$

Since $\left|\varepsilon \eta^{\varepsilon}(s, \cdot)\right|_{\Sigma} \leq C\left(\alpha, \mu_{0}\right)$ for $s \leq \tau^{\varepsilon} \wedge T$,

$$
\begin{aligned}
& \mathbb{E}\left(\sup _{t \leq \tilde{\tau}^{\varepsilon} \wedge T} \int_{0}^{t}\left|D_{3}(s)\right| d s\right) \leq C\left(\sigma, \mu_{0}, \alpha\right)\left(\mathbb{E}\left(\int_{0}^{T} \sup _{s \leq \tilde{\tau}^{\varepsilon} \wedge T}\left|\eta^{\varepsilon}(s)\right|_{\Sigma}^{2}\right)+T\right) \\
& \mathbb{E}\left(\sup _{t \leq \tau^{\varepsilon} \wedge T} \int_{0}^{t}\left|D_{5}(s)\right| d s\right) \leq C\left(\sigma, \mu_{0}, \alpha\right) T .
\end{aligned}
$$

A use of martingale inequality leads to

$$
\begin{aligned}
\mathbb{E}\left(\sup _{t \leq \tilde{\tau}^{\varepsilon} \wedge T}\left|\int_{0}^{t} M_{2}(s) d W(s)\right|\right) \leq & 24 \varepsilon T^{1 / 2} \mathbb{E}\left(\sup _{t \leq \tilde{\tau}^{\varepsilon} \wedge T}\left|\eta^{\varepsilon}(t)\right|_{\Sigma}^{2}\right) \\
& +C\left(\mu_{0}, \alpha, T\right) \mathbb{E}\left(\sup _{t \leq \tilde{\tau}^{\varepsilon} \wedge T}\left|\eta^{\varepsilon}(t)\right|_{L^{2}}^{2}\right)+C\left(T, \mu_{0}\right) .
\end{aligned}
$$

Therefore, if we get

$$
\mathbb{E}\left(\sup _{t \leq \tilde{\tau}^{\complement} \wedge T} \int_{0}^{t}\left|D_{4}(s)\right| d s\right) \leq C\left(\sigma, \mu_{0}, \alpha, N\right) \mathbb{E}\left(\int_{0}^{T} \sup _{s \leq \tilde{\tau}^{\varepsilon} \wedge T}\left|\eta^{\varepsilon}(s)\right|_{\Sigma}^{2}\right),
$$

noting that for some $\nu>0$

$$
\left(L_{\mu_{0}}^{-} \eta_{R}^{\varepsilon}, \eta_{R}^{\varepsilon}\right)+\left(L_{\mu_{0}}^{+} \eta_{I}^{\varepsilon}, \eta_{I}^{\varepsilon}\right) \geq \nu\left(\left|\eta_{R}^{\varepsilon}\right|_{\Sigma}^{2}+\left|\eta_{I}^{\varepsilon}\right|_{\Sigma}^{2}\right)
$$

the estimate

$$
\mathbb{E}\left(\sup _{t \leq \tilde{\tau}^{\varepsilon} \wedge T}\left|\eta^{\varepsilon}(t)\right|_{\Sigma}^{2}\right) \leq C\left(\alpha, \mu_{0}, T, N\right)
$$

will hold, similarly to the proof of Lemma 5.1 (i), choosing $\varepsilon$ small enough. Then, using again Itô formula for $\left|\eta^{\varepsilon}(t)\right|_{\Sigma}^{4}$, since

$d\left|\eta^{\varepsilon}\right|_{\Sigma}^{2}=\{$ the right hand side of $(7.3)\}+\left(1-\mu_{0}\right) d\left|\eta^{\varepsilon}\right|_{L^{2}}^{2}+2(2 \sigma+1)\left(\phi_{\mu_{0}}^{2 \sigma} \eta_{R}^{\varepsilon}, d \eta_{R}^{\varepsilon}\right) d t$

$$
\begin{aligned}
& +\left(\phi_{\mu_{0}}^{2 \sigma}\left(-b^{\varepsilon} \partial_{\mu} \phi_{\mu^{\varepsilon}}+\varepsilon|x|^{2} \eta_{I}^{\varepsilon}+\varepsilon z^{\varepsilon} \eta_{I}^{\varepsilon}\right),-b^{\varepsilon} \partial_{\mu} \phi_{\mu^{\varepsilon}}+\varepsilon|x|^{2} \eta_{I}^{\varepsilon}+\varepsilon z^{\varepsilon} \eta_{I}^{\varepsilon}\right) d t+2\left(\phi_{\mu_{0}}^{2 \sigma} \eta_{I}^{\varepsilon}, d \eta_{I}^{\varepsilon}\right) d t \\
& +\left(\phi_{\mu_{0}}^{2 \sigma}\left(|x|^{2} \phi_{\mu^{\varepsilon}}+z^{\varepsilon} \phi_{\mu^{\varepsilon}}+\varepsilon|x|^{2} \eta_{R}^{\varepsilon}+\varepsilon z^{\varepsilon} \eta_{R}^{\varepsilon}\right),|x|^{2} \phi_{\mu^{\varepsilon}}+z^{\varepsilon} \phi_{\mu^{\varepsilon}}+\varepsilon|x|^{2} \eta_{R}^{\varepsilon}+\varepsilon z^{\varepsilon} \eta_{R}^{\varepsilon}\right) d t,
\end{aligned}
$$

we may write, for $t \leq \tilde{\tau}^{\varepsilon} \wedge T=\tau$,

$$
\left|\eta^{\varepsilon}(t)\right|_{\Sigma}^{4} \leq C \int_{0}^{\tau \wedge t}\left(1+\left|\eta^{\varepsilon}(s)\right|_{\Sigma}^{4}\right) d s+\left|\int_{0}^{\tau \wedge t} A(s) d W(s)\right|
$$


with

$$
|A(s)| \leq C^{\prime}\left(1+\left|\eta^{\varepsilon}\right|_{L^{2}}^{2}\right)\left|\eta^{\varepsilon}\right|_{\Sigma}^{2}+\varepsilon C^{\prime \prime}\left|\eta^{\varepsilon}\right|_{\Sigma}^{4}, \quad \text { a.s. } \quad \text { for } \quad s \leq \tau \text {. }
$$

We then conclude using Lemma 5.1 (ii), and $\varepsilon$ small enough.

We now study in details $D_{4}(t)=-2 \varepsilon\left(L_{\mu_{0}}^{-} \eta_{R}^{\varepsilon}, h_{I}^{\varepsilon}\right)+2 \varepsilon\left(L_{\mu_{0}}^{+} \eta_{I}^{\varepsilon}, h_{R}^{\varepsilon}\right)$; this is divided into

$$
\begin{aligned}
D_{4}(t)= & -2 \varepsilon\left(\nabla \eta_{R}^{\varepsilon}, \nabla h_{I}^{\varepsilon}\right)+2 \varepsilon\left(\nabla \eta_{I}^{\varepsilon}, \nabla h_{R}^{\varepsilon}\right)-2 \varepsilon\left(|x|^{2} \eta_{R}^{\varepsilon}, h_{I}^{\varepsilon}\right)+2 \varepsilon\left(|x|^{2} \eta_{I}^{\varepsilon}, h_{R}^{\varepsilon}\right) \\
& -2 \varepsilon \mu_{0}\left(\eta_{R}^{\varepsilon}, h_{I}^{\varepsilon}\right)+2 \varepsilon \mu_{0}\left(\eta_{I}^{\varepsilon}, h_{R}^{\varepsilon}\right)+2 \varepsilon(2 \sigma+1)\left(\phi_{\mu_{0}}^{2 \sigma} \eta_{R}^{\varepsilon}, h_{I}^{\varepsilon}\right)-2 \varepsilon\left(\phi_{\mu_{0}}^{2 \sigma} \eta_{I}^{\varepsilon}, h_{R}^{\varepsilon}\right) .
\end{aligned}
$$

In order to estimate these terms in $d=2$, we need the stopping time $\bar{\tau}_{N}^{\varepsilon}$ which allows us to make use of the Sobolev embedding $\Sigma^{2} \subset L^{\infty}\left(\mathbb{R}^{2}\right)$. The case $d=1$ is easier, so we give here a proof for $d=2$. Using $\left|\varepsilon \eta^{\varepsilon}(s, \cdot)\right|_{\Sigma} \leq C\left(\mu_{0}, \alpha\right)$ for $t \leq \tau^{\varepsilon} \wedge T$, and

$$
\left|\varepsilon h^{\varepsilon}\right|_{L^{2}} \leq C\left|\varepsilon \eta^{\varepsilon}\right|_{L^{4 \sigma+4}}^{2 \sigma}\left|\eta^{\varepsilon}\right|_{L^{2 \sigma+2}} \leq C\left(\alpha, \mu_{0}, \sigma\right)\left|\eta^{\varepsilon}\right|_{\Sigma}, \quad t \leq \tau^{\varepsilon} \wedge T,
$$

we have

$$
\begin{array}{r}
\left|-2 \varepsilon \mu_{0}\left(\eta_{R}^{\varepsilon}, h_{I}^{\varepsilon}\right)+2 \varepsilon \mu_{0}\left(\eta_{I}^{\varepsilon}, h_{R}^{\varepsilon}\right)+2 \varepsilon(2 \sigma+1)\left(\phi_{\mu_{0}}^{2 \sigma} \eta_{R}^{\varepsilon}, h_{I}^{\varepsilon}\right)-2 \varepsilon\left(\phi_{\mu_{0}}^{2 \sigma} \eta_{I}^{\varepsilon}, h_{R}^{\varepsilon}\right)\right| \\
\leq C\left(\mu_{0}, \sigma\right)\left|\eta^{\varepsilon}\right|_{L^{2}}\left|\varepsilon h^{\varepsilon}\right|_{L^{2}} \leq C\left(\mu_{0}, \sigma, \alpha\right)\left|\eta^{\varepsilon}\right|_{L^{2}}\left|\eta^{\varepsilon}\right|_{\Sigma}, \quad t \leq \tau^{\varepsilon} \wedge T .
\end{array}
$$

In the same way, we obtain also

$$
\left|-2 \varepsilon\left(|x|^{2} \eta_{R}^{\varepsilon}, h_{I}^{\varepsilon}\right)+2 \varepsilon\left(|x|^{2} \eta_{I}^{\varepsilon}, h_{R}^{\varepsilon}\right)\right| \leq C\left(\mu_{0}, \alpha, \sigma\right)\left|\eta^{\varepsilon}\right|_{\Sigma}^{2}, \quad t \leq \tau^{\varepsilon}
$$


We perform the calculations of $\left|2 \varepsilon\left(\nabla \eta_{I}^{\varepsilon}, \nabla h_{R}^{\varepsilon}\right)\right|$ since the other term is treated similarly. We develop here the derivative of $h_{R}^{\varepsilon}$ :

$$
\begin{aligned}
\nabla h_{R}^{\varepsilon}= & \frac{2 \sigma}{\varepsilon^{2}} \int_{0}^{1}(1-s)(\sigma-1)\left[\left(\phi_{\mu^{\varepsilon}}+s \varepsilon \eta_{R}^{\varepsilon}\right)^{2}+\left(s \varepsilon \eta_{I}^{\varepsilon}\right)^{2}\right]^{\sigma-2} \\
& \times\left\{2\left(\phi_{\mu^{\varepsilon}}+s \varepsilon \eta_{R}^{\varepsilon}\right)\left(\nabla \phi_{\mu^{\varepsilon}}+s \varepsilon \nabla \eta_{R}^{\varepsilon}\right)+2 s^{2} \varepsilon^{2} \eta_{I}^{\varepsilon} \nabla \eta_{I}^{\varepsilon}\right\} \\
& \times\left\{2\left(\left(\phi_{\mu^{\varepsilon}}+s \varepsilon \eta_{R}^{\varepsilon}\right) \varepsilon \eta_{R}^{\varepsilon}+s\left(\varepsilon \eta_{I}^{\varepsilon}\right)^{2}\right) \varepsilon \eta_{R}^{\varepsilon}+\left(\left(\varepsilon \eta_{R}^{\varepsilon}\right)^{2}+\left(\varepsilon \eta_{I}^{\varepsilon}\right)^{2}\right)\left(\phi_{\mu^{\varepsilon}}+s \varepsilon \eta_{R}^{\varepsilon}\right)\right\} d s \\
& +\frac{2 \sigma}{\varepsilon^{2}} \int_{0}^{1}(1-s)\left[\left(\phi_{\mu^{\varepsilon}}+s \varepsilon \eta_{R}^{\varepsilon}\right)^{2}+\left(s \varepsilon \eta_{I}^{\varepsilon}\right)^{2}\right]^{\sigma-1} \\
& \times\left\{2\left(\left(\phi_{\mu^{\varepsilon}}+s \varepsilon \eta_{R}^{\varepsilon}\right) \varepsilon \eta_{R}^{\varepsilon}+s\left(\varepsilon \eta_{I}^{\varepsilon}\right)^{2}\right) \varepsilon \nabla \eta_{R}^{\varepsilon}\right. \\
& +2\left(\left(\nabla \phi_{\mu^{\varepsilon}}+s \varepsilon \nabla \eta_{R}^{\varepsilon}\right) \varepsilon \eta_{R}^{\varepsilon}+\left(\phi_{\mu^{\varepsilon}}+s \varepsilon \eta_{R}^{\varepsilon}\right) \varepsilon \nabla \eta_{R}^{\varepsilon}+2 s \varepsilon^{2} \eta_{I}^{\varepsilon} \nabla \eta_{I}^{\varepsilon}\right) \varepsilon \eta_{R}^{\varepsilon} \\
& \left.+\left(\left(\varepsilon \eta_{R}^{\varepsilon}\right)^{2}+\left(\varepsilon \eta_{I}^{\varepsilon}\right)^{2}\right)\left(s \varepsilon \nabla \eta_{R}^{\varepsilon}+\nabla \phi_{\mu^{\varepsilon}}\right)+\left(2 \varepsilon^{2} \eta_{R}^{\varepsilon} \nabla \eta_{R}^{\varepsilon}+2 \varepsilon^{2} \eta_{I}^{\varepsilon} \nabla \eta_{I}^{\varepsilon}\right)\left(\phi_{\mu^{\varepsilon}}+s \varepsilon \eta_{R}^{\varepsilon}\right)\right\} d s \\
& +\frac{4 \sigma(\sigma-1)}{\varepsilon^{2}} \int_{0}^{1}(1-s)(\sigma-2)\left[\left(\phi_{\mu^{\varepsilon}}+s \varepsilon \eta_{R}^{\varepsilon}\right)^{2}+\left(s \varepsilon \eta_{I}^{\varepsilon}\right)^{2}\right]^{\sigma-3} \\
& \times\left\{2\left(\phi_{\mu^{\varepsilon}}+s \varepsilon \eta_{R}^{\varepsilon}\right)\left(\nabla \phi_{\mu^{\varepsilon}}+s \varepsilon \nabla \eta_{R}^{\varepsilon}\right)+2 s^{2} \varepsilon^{2} \eta_{I}^{\varepsilon} \nabla \eta_{I}^{\varepsilon}\right\} \\
& \times\left(\left(\phi_{\mu^{\varepsilon}}+s \varepsilon \eta_{R}^{\varepsilon}\right) \varepsilon \eta_{R}^{\varepsilon}+s\left(\varepsilon \eta_{I}^{\varepsilon}\right)^{2}\right)^{2}\left(\phi_{\mu^{\varepsilon}}+s \varepsilon \eta_{R}^{\varepsilon}\right) d s \\
& +\frac{4 \sigma(\sigma-1)}{\varepsilon^{2}} \int_{0}^{1}(1-s)\left[\left(\phi_{\mu^{\varepsilon}}+s \varepsilon \eta_{R}^{\varepsilon}\right)^{2}+\left(s \varepsilon \eta_{I}^{\varepsilon}\right)^{2}\right]^{\sigma-2} \\
& \times\left\{\left(\left(\phi_{\mu^{\varepsilon}}+s \varepsilon \eta_{R}^{\varepsilon}\right) \varepsilon \eta_{R}^{\varepsilon}+s\left(\varepsilon \eta_{I}^{\varepsilon}\right)^{2}\right)^{2}\left(\nabla \phi_{\mu^{\varepsilon}}+s \varepsilon \nabla \eta_{R}^{\varepsilon}\right)+2\left(\left(\phi_{\mu^{\varepsilon}}+s \varepsilon \eta_{R}^{\varepsilon}\right) \varepsilon \eta_{R}^{\varepsilon}+s\left(\varepsilon \eta_{I}^{\varepsilon}\right)^{2}\right)\right. \\
& \left.\left(\left(\phi_{\mu^{\varepsilon}}+s \varepsilon \eta_{R}^{\varepsilon}\right) \varepsilon \nabla \eta_{R}^{\varepsilon}+\left(\nabla \phi_{\mu^{\varepsilon}}+s \varepsilon \nabla \eta_{R}^{\varepsilon}\right) \varepsilon \eta_{R}^{\varepsilon}+2 s \varepsilon^{2} \eta_{I}^{\varepsilon} \nabla \eta_{I}^{\varepsilon}\right)\left(\phi_{\mu^{\varepsilon}}+s \varepsilon \eta_{R}^{\varepsilon}\right)\right\} d s
\end{aligned}
$$

We estimate the term, as an example, $\frac{2 \sigma}{\varepsilon^{2}}\left|\left(\nabla \eta_{I}^{\varepsilon}, A \varepsilon \eta_{R}^{\varepsilon}\right)\right|$ where

$$
\begin{aligned}
A= & \int_{0}^{1}(1-s)(\sigma-1)\left[\left(\phi_{\mu^{\varepsilon}}+s \varepsilon \eta_{R}^{\varepsilon}\right)^{2}+\left(s \varepsilon \eta_{I}^{\varepsilon}\right)^{2}\right]^{\sigma-2} \\
& \times\left\{2\left(\phi_{\mu^{\varepsilon}}+s \varepsilon \eta_{R}^{\varepsilon}\right)\left(\nabla \phi_{\mu^{\varepsilon}}+s \varepsilon \nabla \eta_{R}^{\varepsilon}\right)+2 s^{2} \varepsilon^{2} \eta_{I}^{\varepsilon} \nabla \eta_{I}^{\varepsilon}\right\} \\
& \times 2\left(\left(\phi_{\mu^{\varepsilon}}+s \varepsilon \eta_{R}^{\varepsilon}\right) \varepsilon \eta_{R}^{\varepsilon}+2 s\left(\varepsilon \eta_{I}^{\varepsilon}\right)^{2}\right) d s .
\end{aligned}
$$


It is majorized for $t \leq \tau^{\varepsilon} \wedge \bar{\tau}_{N}^{\varepsilon} \wedge T$, assuming $\sigma \geq 1$, as follows;

$$
\begin{aligned}
\frac{2 \sigma}{\varepsilon^{2}}\left|\left(\nabla \eta_{I}^{\varepsilon}, A \varepsilon \eta_{R}^{\varepsilon}\right)\right| \leq & C\left(\left|\phi_{\mu^{\varepsilon}}\right|_{L^{\infty}}+\left|\nabla \phi_{\mu^{\varepsilon}}\right|_{L^{\infty}}\right)\left|\phi_{\mu^{\varepsilon}}\right|_{L^{\infty}}^{2(\sigma-1)}\left|\nabla \eta^{\varepsilon}\right|_{L^{2}}\left|\eta^{\varepsilon}\right|_{L^{2}} \\
& +C\left(\left|\phi_{\mu^{\varepsilon}}\right|_{L^{\infty}}+\left|\nabla \phi_{\mu^{\varepsilon}}\right|_{L^{\infty}}\right) \int_{\mathbb{R}^{d}}\left|\nabla \eta^{\varepsilon}\right|\left|\varepsilon \eta^{\varepsilon}\right|^{2(\sigma-1)}\left|\eta^{\varepsilon}\right| \\
& +\frac{C}{\varepsilon}\left|\phi_{\mu^{\varepsilon}}\right|_{L^{\infty}}^{2(\sigma-1)} \int_{\mathbb{R}^{d}}\left|\nabla \eta^{\varepsilon}\right|\left|\varepsilon \eta^{\varepsilon}\right|\left|\varepsilon \nabla \eta^{\varepsilon}\right|+\frac{C}{\varepsilon} \int_{\mathbb{R}^{d}}\left|\nabla \eta^{\varepsilon}\right|\left|\varepsilon \eta^{\varepsilon}\right|^{2 \sigma-1}\left|\varepsilon \nabla \eta^{\varepsilon}\right| \\
& +\frac{C}{\varepsilon}\left|\phi_{\mu^{\varepsilon}}\right|_{L^{\infty}}^{2(\sigma-1)} \int_{\mathbb{R}^{d}}\left|\nabla \eta^{\varepsilon}\right|\left|\varepsilon \eta^{\varepsilon}\right|^{2}+\frac{C}{\varepsilon} \int_{\mathbb{R}^{d}}\left|\nabla \eta^{\varepsilon}\right|\left|\varepsilon \eta^{\varepsilon}\right|^{2 \sigma} \\
& \leq C\left(\mu_{0}, \alpha, N\right)\left(\left|\nabla \eta^{\varepsilon}\right|_{L^{2}}\left|\eta^{\varepsilon}\right|_{L^{2}}+\left|\nabla \eta^{\varepsilon}\right|_{L^{2}}^{2}\right),
\end{aligned}
$$

where in the last inequality we have used

$$
\left|\varepsilon \eta^{\varepsilon}\right|_{L^{\infty}} \leq C\left|\varepsilon \eta^{\varepsilon}\right|_{\Sigma^{2}} \leq N, \quad t \leq \tau^{\varepsilon} \wedge \bar{\tau}_{N}^{\varepsilon} \wedge T .
$$

We remark that in case of $d=1$ we can simply benefit from

$$
\left|\varepsilon \eta^{\varepsilon}\right|_{L^{\infty}} \leq C\left|\varepsilon \eta^{\varepsilon}\right|_{\Sigma} \leq C\left(\mu_{0}, \alpha\right), \quad t \leq \tau^{\varepsilon} \wedge T .
$$

Other terms are also estimated as above and then we may prove (7.4) where we replace $\tau^{\varepsilon}$ with $\tau^{\varepsilon} \wedge \bar{\tau}_{N}^{\varepsilon}$ in $d=2$.

Proof of Lemma $5.3(i)$. This estimate may be proved similarly to the proof of Lemma 5.1 (i), it is sufficient to mention the following; we have, recalling that $\eta$ satisfies Eq. (5.1),

$$
d\left(\left|\eta_{R}\right|_{L^{2}}^{2}+\left|\eta_{I}\right|_{L^{2}}^{2}\right)=4 \sigma\left(\eta_{R}, \phi_{\mu_{0}}^{2 \sigma} \eta_{I}\right) d t-2\left(\eta_{I},|x|^{2} \phi_{\mu_{0}}\right) d W+\left.\left.|| x\right|^{2} \phi_{\mu_{0}}\right|_{L^{2}} ^{2} d t-\frac{\left|x \phi_{\mu_{0}}\right|_{L^{2}}^{4}}{\left|\phi_{\mu_{0}}\right|_{L^{2}}^{2}} d t
$$

by Itô formula.

Proof of Lemma 5.3 (ii). We apply Itô formula to $\left(L_{\mu_{0}}^{-} \eta_{R}, \eta_{R}\right)+\left(L_{\mu_{0}}^{+} \eta_{I}, \eta_{I}\right)$, then we have

$$
d\left(\left(L_{\mu_{0}}^{-} \eta_{R}, \eta_{R}\right)+\left(L_{\mu_{0}}^{+} \eta_{I}, \eta_{I}\right)\right)=\left(L_{\mu_{0}}^{+}\left(|x|^{2} \phi_{\mu_{0}}\right),|x|^{2} \phi_{\mu_{0}}\right) d t-2\left(\eta_{I}, L_{\mu_{0}}^{+}\left(|x|^{2} \phi_{\mu_{0}}\right)\right) d W .
$$

Therefore,

$$
\begin{aligned}
\mathbb{E}\left(\sup _{t \leq T}\left(\left(L_{\mu_{0}}^{-} \eta_{R}, \eta_{R}\right)+\left(L_{\mu_{0}}^{+} \eta_{I}, \eta_{I}\right)\right)\right) & \leq C T^{1 / 2}\left(\mathbb{E}\left(\sup _{t \leq T}\left|\eta_{I}(t)\right|_{L^{2}}^{2}\right)\right)^{1 / 2}+C^{\prime} T \\
& \leq \frac{\nu}{2} \mathbb{E}\left(\sup _{t \leq T}\left|\eta_{I}(t)\right|_{L^{2}}^{2}\right)+C T
\end{aligned}
$$

where $\nu$ is given by

$$
\left(L_{\mu_{0}}^{-} \eta_{R}, \eta_{R}\right)+\left(L_{\mu_{0}}^{+} \eta_{I}, \eta_{I}\right) \geq \nu\left(\left|\eta_{R}\right|_{\Sigma}^{2}+\left|\eta_{I}\right|_{\Sigma}^{2}\right),
$$

which holds since $\eta_{R}$ and $\eta_{I}$ satisfy $\left(\eta_{R}, \phi_{\mu_{0}}\right)=0$ and $\left(\eta_{I}, \phi_{\mu_{0}}\right)=0$. Accordingly, we obtain

$$
\mathbb{E}\left(\sup _{t \leq T}\left(\left|\eta_{R}(t)\right|_{\Sigma}^{2}+\left|\eta_{I}(t)\right|_{\Sigma}^{2}\right)\right) \leq C T .
$$

Note that this constant $C$ depends only on $\mu_{0}, \nu$. Hence the right hand side is bounded linearly in $T$ which shows (2.8). 
Proof of Lemma 5.3 (iii). Itô formula for $\left|\left(1+|x|^{4}\right) \eta\right|_{L^{2}}^{2}$ implies

$$
\begin{aligned}
d\left|\left(1+|x|^{4}\right) \eta_{R}\right|_{L^{2}}^{2}= & 2\left(\left(1+|x|^{4}\right) \eta_{R}, L_{\mu_{0}}^{+} \eta_{I}\right) d t, \\
d\left|\left(1+|x|^{4}\right) \eta_{I}\right|_{L^{2}}^{2}= & -2\left(\left(1+|x|^{4}\right) \eta_{I}, L_{\mu_{0}}^{-} \eta_{R}\right) d t+2 \frac{\left(L_{\mu_{0}}^{-} \eta_{R}, \phi_{\mu_{0}}\right)}{\left|\phi_{\mu_{0}}\right|_{L^{2}}^{2}}\left(\phi_{\mu_{0}},\left(1+|x|^{4}\right) \eta_{I}\right) d t \\
& -2\left(\left(1+|x|^{4}\right) \eta_{I},|x|^{2} \phi_{\mu_{0}}\right) d W+2 \frac{\left(\left(1+|x|^{4}\right) \eta_{I}, \phi_{\mu_{0}}\right)}{\left|\phi_{\mu_{0}}\right|_{L^{2}}^{2}\left|x \phi_{\mu_{0}}\right|_{L^{2}}^{2}} d W \\
& +\left|\left(1+|x|^{4}\right)^{1 / 2}\left(-|x|^{2} \phi_{\mu_{0}}+\frac{\left|x \phi_{\mu_{0}}\right|_{L^{2}}^{2} \phi_{\mu_{0}}}{\left|\phi_{\mu_{0}}\right|_{L^{2}}^{2}}\right)\right|_{L^{2}}^{2} d t .
\end{aligned}
$$

We here note that

$$
\begin{aligned}
& \left(\left(1+|x|^{4}\right) \eta_{R}, L_{\mu_{0}}^{+} \eta_{I}\right)-\left(\left(1+|x|^{4}\right) \eta_{I}, L_{\mu_{0}}^{-} \eta_{R}\right) \\
& =-4(2+d)\left(|x|^{2} \eta_{R}, \eta_{I}\right)-8\left(|x|^{2} x \cdot \nabla \eta_{R}, \eta_{I}\right)+2 \sigma\left(\left(1+|x|^{4}\right) \eta_{I}, \phi_{\mu_{0}}^{2 \sigma} \eta_{R}\right) .
\end{aligned}
$$

Hence,

$$
d\left|\left(1+|x|^{4}\right) \eta\right|_{L^{2}}^{2}=D(t) d t+M(t) d W(t)
$$

with

and

$$
|D(t)| \leq C\left|\left(1+|x|^{4}\right) \eta\right|_{L^{2}}^{2}+C^{\prime}\left(1+|\eta|_{\Sigma}^{2}\right)
$$

$$
|M(t)| \leq C\left|\left(1+|x|^{4}\right) \eta_{I}\right|_{L^{2}}+C^{\prime}
$$

Similarly as above, Gronwall lemma, a martingale inequality and the estimates proved in Lemma 5.3 (ii) allow to conclude

$$
\mathbb{E}\left(\sup _{t \leq T}\left|\left(1+|x|^{4}\right) \eta(t)\right|_{L^{2}}^{2}\right) \leq C(T) .
$$

\section{REFERENCES}

1. F.Kh. Abdullaev, B.B Baizakov and V.V. Konotop, "Dynamics of a Bose-Einstein condensate in optical trap," in: Nonlinearity and Disorder : Theory and Applications, edited by F.Kh. Abdullaev, O. Bang and M.P. Soerensen, NATO Science Series vol. 45, Kluwer Dodrecht, 2001.

2. F.Kh. Abdullaev, J.C. Bronski and G. Papanicolaou, "Soliton perturbations and the random Kepler problem," Physica D 135 (2000) 369-386.

3. R. Carles, "Remarks on nonlinear Schrödinger equations with harmonic potential," Ann. Henri Poincaré. 3 (2002) 757-772.

4. T. Cazenave and P. L. Lions, "Orbital stability of standing waves for some nonlinear Schrödinger equations," Commun. Math. Phys. 85 (1982) 549-561.

5. G. Da Prato and J. Zabczyk, "Stochastic equations in infinite dimensions," Encyclopedia of Mathematics and its Applications; Cambridge University Press: Cambridge, England, 1992.

6. A. de Bouard and A. Debussche, "Random modulation of solitons for the stochastic Korteweg-de Vries equation," Ann. I. H. Poincaré. 24 (2007) 251-278.

7. A. de Bouard and A. Debussche, "Dynamics of solitons for the Korteweg-de Vries equation with multiplicative homogeneous noise" Preprint.

8. A. de Bouard and R. Fukuizumi, "Stochastic fluctuations in the Gross-Pitaevskii equation," Nonlinearity 20 (2007) 2821-2844. 
9. A. de Bouard and E. Gautier, "Exit problems related to the persistence of solitons for the Korteweg-de Vries equation with small noise," Preprint.

10. R. Fukuizumi and M.Ohta, "Stability of standing waves for nonlinear Schrödinger equations with potentials," Differential and Integral Equations. 16 (2003) 111-128.

11. R. Fukuizumi and T. Ozawa, "Exponential decay of solutions to nonlinear elliptic equations with potentials," Z. Angew. Math. Phys. 56 (2005) 1000-1011.

12. J. Garnier, F.Kh. Abdullaev and B.B. Baizakov, "Collapse of a Bose-Einstein condensate induced by fluctuations of the laser intensity," Phys. Rev. A 69 (2004) 053607, 369-386.

13. M. Grillakis, "Linearized instability for nonlinear Schrödinger and Klein-Gordon equations," Commun. Pure Appl. Math. 41 (1988) 747-774.

14. M. Grillakis, J. Shatah and W. Strauss, "Stability theory of solitary waves in the presence of symmetry I,II," J. Funct. Anal. Part I: 74 (1987) 160-197, Part II: 94 (1990) 308-348.

15. B. L. Jonsson, J. Fröhlich, S. Gustafson and I. M. Sigal, "Solitary wave dynamics in an external potential," Commun. Math. Phys. 250 (2004) 613-642.

16. B. L. Jonsson, J. Fröhlich, S. Gustafson and I. M. Sigal, "Long time motion of NLS solitary waves in a confining potential," Ann. Henri Poincaré. 7 (2006) 621-660.

17. Y. Kabeya and K. Tanaka, "Uniqueness of positive radial solutions of semilinear elliptic equations in $\mathbb{R}^{N}$ and Séré's non-degeneracy condition,". Comm. Partial Differential Equations. 24 (1999) 563-598.

18. Kirr and M. I. Weinstein, "Diffusion of power in randomly perturbed Hamiltonian partial differential equations," Commun. Math. Phys. 255 (2005) 293-328.

19. M. Kurth, "On the existence of infinitely many modes of a nonlocal nonlinear Schrödinger equation related to dispersion-managed solitons," SIAM J. Math. Anal. 36 (2004/05) 967-985.

20. Y. Li and W. N. Ni, "Radial symmetry of positive solutions of nonlinear elliptic equations in $\mathbb{R}^{n}$," Commun. Partial Differential Eqs. 18 (1993) 1043-1054.

21. Y. G. Oh, "Cauchy problem and Ehrenfest's law of nonlinear Schrödinger equations with potentials," J. Differential Equations 81 (1989) 255-274.

22. G. Papanicolaou, "A kinetic theory for power transfer in stochastic systems," J. Math. Phys. 13 (1972) 1912-1918.

23. D. E. Pelinovsky and P.G. Kevrekidis, "Periodic oscillations of dark solitons in parabolic potentials," Preprint.

24. L. P. Pitaevskii and S. Stringari, "Bose-Einstein condensation," Oxford Univ. Press, New York (2003)

25. M. Reed and B. Simon, "Method of modern mathematical physics, I,II,III,IV" Academic Press, 1975.

26. H. A. Rose and M. I. Weinstein, "On the bound states of the nonlinear Schrödinger equation with a linear potential," Physica D. 30 (1988) 207-218.

27. J. Shatah and W. Strauss, "Instability of nonlinear bound states," Commun. Math. Phys. 100 (1985) 173-190.

28. D. M. Stamper-Kurn, M. R. Andrews, A. P. Chikkatur, S. Inouye, H. J. Miesner, J. Stenger and W. Ketterle, "Optical confinement of a Bose-Einstein condensate," Phys. Rev. Lett. 80 (1998) 2027-2030.

29. E.C. Titchmarsh, "Eigenfunction expansions; associated with second-order differential equations, Part I" Second edition, Oxford at the Clarendon press, 1962.

30. M. I. Weinstein, "Modulational stability of ground states of nonlinear Schrödinger equations," SIAM J. Math. Anal. 16 (1985) 472-491.

31. M. I. Weinstein, "Lyapunov stability of ground states of nonlinear dispersive evolution equations," Commun. Pure Appl. Math. 39 (1986) 51-67. 